\title{
Magnetosphere-Ionosphere Convection as a Compound System
}

\author{
T. Tanaka
}

Received: 9 February 2007 / Accepted: 22 February 2007 /

Published online: 1 June 2007

(C) Springer Science+Business Media, Inc. 2007

\begin{abstract}
Convection is the most fundamental process in understanding the structure of geospace and disturbances observed in the magnetosphere-ionosphere (M-I) system. In this paper, a self-consistent configuration of the global convection system is considered under the real topology as a compound system. Investigations are made based on the M-I coupling scheme by analyzing numerical results obtained from magnetohydrodynamic (MHD) simulations which guarantee the self-consistency in the whole system under the Bv (magnetic field and velocity) paradigm. It is emphasized in the M-I coupling scheme that convection and field-aligned current (FAC) are different aspects of same physical process characterizing the open magnetosphere. Special attention is given in this paper to the energy supplying (dynamo) process that drives the FAC system. In the convection system, the dynamo must be constructed from shear motion together with plasma population regimes to steadily drive the convection. Convection patterns observed in the ionosphere are also the manifestation of achievement in global self-consistency. A primary approach to apply these concepts to the study of geospace is to consider how the M-I system adjusts the relative motion between the compressible magnetosphere and the incompressible ionosphere when responding to given solar-wind conditions. The above principle is also applicable for the study of disturbance phenomena such as the substorm as well as for the study of apparently unique processes such as the flux transfer event (FTE), the sudden commencement (SC), and the theta aurora. Finally, an attempt is made to understand the substorm as the extension of enhanced convection under the southward interplanetary magnetic field (IMF) condition.
\end{abstract}

Keywords Convection · Field-aligned current $\cdot$ Dynamo $\cdot$ Plasma regimes · MHD simulation

T. Tanaka (凶)

Faculty of Earth and Planetary Sciences, Graduated School of Sciences, Kyushu University,

Fukuoka 812-8581, Japan

e-mail: tatanaka@geo.kyushu-u.ac.jp

T. Tanaka

CREST, Japan Science and Technology Agency, Kawaguchi 332-0012, Japan 


\section{Introduction}

Past the Parker's singular point, most of the internal energy of the solar wind is converted into kinetic energy, and from there on, kinetic energy becomes dominant in the solar wind (Parker 1958). The confinement of the Earth's magnetic field by this dynamic pressure of the solar wind acting from one side is the primary reason for the formation of the cometshaped magnetosphere. Chapman and Ferraro (1931) established these foundations of magnetospheric formation. In ideal magnetohydrodynamic (MHD) processes under the frozen-in principle

$$
\mathbf{E}+\mathbf{v} \times \mathbf{B}=0,
$$

for electric field $\mathbf{E}$, velocity $\mathbf{v}$, and magnetic field $\mathbf{B}$, the solar wind plasma and magnetospheric plasma (if present) would not mix, resulting in a quiet magnetosphere. In such a case, the magnetopause would represent a tangential discontinuity that transfers only the ram force to the earth (Siscoe 1966). This magnetosphere produced by diamagnetic plasma can be called the closed magnetosphere. It is a quiet magnetosphere, which generates neither the aurora nor substorms, because it does not permit the penetration of solar wind energy into the magnetosphere (Akasofu 1996). The process by which the magnetosphere deviates from being an absolutely quiet magnetosphere is the magnetospheric convection associated with the plasma penetration.

It is well known that the concept of convection starts with Dungey (1961) and Axford and Hines (1961). Of the two, Dungey's model had gotten wide support and is still adopted by many researchers as a basic concept of magnetospheric dynamics. In these models, convection is excited through non-ideal MHD processes such as reconnection and magnetopause instability. In these considerations, however, the whole system driving the magnetosphere-ionosphere (M-I) convection were not yet organized in a self-consistent manner. The present paper tries to advance this problem by reconsidering the self-consistent configuration of $\mathrm{M}-\mathrm{I}$ convection under a realistic topology, including the field-aligned currents (FACs), ionospheric currents, magnetospheric dynamo process, and plasma population regimes in the magnetosphere (Tanaka 2003b), to show that simultaneous plasma penetration into the magnetosphere and resulting plasma regimes are indispensable to establish the self-consistency in the convection system in the solar wind-magnetosphere-ionosphere ( $\mathrm{S}-$ $\mathrm{M}-\mathrm{I}$ ) interaction process. In this paper, the system that pursues the global self-consistency under a real topology is called a compound system. Through this approach, the consideration of how to realize the requirement to fulfill the global self-consistency in the M-I convection system also enables the unified understanding of electromagnetic structure and disturbance phenomena in the S-M-I system. By these studies, we can obtain a deep understanding of causal relations for many types of disturbances observed in the S-M-I system including substorm. As a consequence, the magnetospheric model of the next level will be revealed through these considerations.

\subsection{Convecting Magnetosphere}

In the early stage of the former century, the primary focus of auroral physics had been the study of why and how the aurora brightens. Before the early 1960s, it had become obvious that molecular processes excited by energetic particles make the auroral brighten. The book by J. Chamberlain was so complete about this problem that there seemed to be nothing left to study on the aurora (Akasofu 1996). However, Akasofu (1964) opened a new door for the auroral physics through the identification of the substorm, by which the main 
target of the research was directed to the clarification of the origin and dynamics of the energetic particles. After the establishment of magnetospheric formation, it had become apparent that disturbance energy comes from the magnetosphere. Then, the auroral physics and the magnetospheric physics were unified as one body. In this view, we must search for the generation mechanisms of auroral energy in the magnetosphere. For this purpose, the electro-dynamical aspect must become conspicuous in the magnetospheric physics. Since the frozen in principle indicates that no convection implies at the same time no electric field, and consequently no ionospheric current, the fundamental process that generates almost all free energies for auroral phenomena and substorms (Akasofu 1964; Elphinstone et al. 1996) must be related with the plasma convection driven by the S-M-I interaction (Vasyliunas 1970; Stern 1977, 1983; Caudal and Blanc 1988; Lyons 1992; Cowley 2000). Thus, understanding of physical process controlling the convection became indispensable in the study of the electro-dynamics of aurora and substorm occurring in the polar ionosphere and magnetosphere. Today, most researchers believe that the convection controls almost all disturbance phenomena occurring in the M-I system as well as the substorm.

Originally, Dungey's model was proposed to explain the SD field of geomagnetic storm defined by Chapman (1935), but afterward it was extensively applied to more general cases of convection. In this model, the geomagnetic field is always exposed to the reconnection with the solar wind magnetic field and not only plasma but also the momentum and energy penetrate into the magnetosphere to induce large-scale convection in the magnetospheric plasma. The magnetosphere interconnected with the interplanetary magnetic field (IMF) is called the open magnetosphere. In this classical model, the magnetic fluxes from the polar region are connected to the IMF fluxes and dragged tailwards over the polar cap by magnetosheath flow. By adopting this convectional concept, magnetosheath flow is supposed to stretch open magnetic fields into a cylindrical tail (Lyons 1992; Cowley 1998). Thus, the convection of lobe magnetic fluxes is mainly controlled by the external force until they reconnect in the plasma sheet. Reconnected flux tubes then contract back toward the earth to form a return path of the convection. However, these traditional understandings of convection do not consider a mechanical force balance exactly and do not directly couple with the formation of plasma population regimes.

An excellent feature of an open magnetosphere is that auroral and magnetic activities inside it are modulated by changes in the IMF to give observed variations in the auroral and substorm occurrences (Fairfield and Cahill 1966). Auroras and much of the radiation belts are generated as a part of convection in the magnetosphere controlled by the IMF. The convection also generates electric currents in accordance with the laws of MHD, creating a large-scale current system in the M-I system. The various changes in the magnetic field observed on ground or in the magnetosphere are attributed to this current system. In the open magnetospheric model, as mentioned above, plasma population regimes are not strictly related to the convection, and the tail magnetopause and the difference between the cusp and the polar cap become rather obscure, at least in their dynamical aspects. However, Dungey's framework for the convection is so splendid that it captivates many researchers even today. This framework already includes the germ of blind belief for the near-earth neutral line (NENL) model of the substorm (see Sect. 7). From the nature of Dungey's convection, it seems quite natural to attribute the expansion phase onset of the substorm to the start of NENL formation at the middle plasma sheet (Baker et al. 1996).

\subsection{Discovery of the FAC}

The large scale FACs were recognized from the observation of low-altitude polar-orbiting satellites (Cumming and Dessler 1967) and the overall morphology was revealed by Iijima 
and Potemra (1976), who referred them as the region-1 and region-2 FACs. The region-1 FAC distributes as a ring surrounding the geomagnetic pole, and consists of a dawn-dusk pair with a down current on the dawnside and an up current on the duskside. The region-2 FAC which lies on the low-latitude side of region-1 FAC has a reverse polarity. Later, two additional FAC systems were also discovered; they were termed dayside cusp system and polar cap NBZ system (Iijima et al. 1984; Burch et al. 1985). These identifications of FAC had given a new insight into the self-consistent structure of the M-I convection system. Recognizing that the meaning of FACs is to represent changes in transverse field as manifestations of Maxwell stresses applied to the ionosphere was a remarkable step to recognize the necessity to consider a coordinated dynamical control for the entire M-I coupling regions including the ionosphere (Cumming and Dessler 1967; Iijima 2000). The magnetospheric physicists realized that the essential role of these large scale FACs is to control the convection in the M-I system by transmitting transverse momentum and stress from the causal region to the other end, and to make the plasma population in a flux tube convect as a whole (Stern 1983; Cowley 2000; Iijima 2000). With the FAC, therefore, magnetospheric convection can proceed with involvement of the ionospheric plasma as well.

While the FACs transmit the magnetic stress, the conducting ionosphere provides a closure path for FAC. Consequently, FACs surely produce magnetic variations on the ground through ionospheric currents joined with or induced by the FAC. From such a point of view, ground features of magnetic field variation studied in traditional geomagnetism can be looked upon as the tracer for FAC closure process in the ionosphere (Akasofu et al. 1965; Obayashi and Nishida 1968; Clauer and Kamide 1985; Kamide et al. 1996; Kikuchi et al. 1996, 2000, 2001; Hashimoto et al. 2002). This closure process is, at the same time, the process to determine the ionospheric convection potential. With this consideration, the high-latitude ionospheric potential extending from the polar cap to the auroral zone is a lowaltitude manifestation of the magnetospheric convection. In the Dungey's picture of global scale merging and convection, the ionospheric convection potential is the mapping of the potential distribution along the dayside reconnection line that is in turn the manifestation of the solar wind potential between outmost reconnecting IMFs (Crooker 1979, 1988).

Unfortunately, Dungey's concept for convection was not extensively altered even after the discovery of the FAC. Since it does not strictly consider the low-beta area in the ionosphere, it does not confront a strict requirement to fully relate the convection with FAC closure processes in the magnetosphere which are coupled to dynamo and plasma distribution in the magnetosphere. As a consequence, it took an unexpectedly long time before we understood how these currents fit in the overall picture, although people had been aware that FACs play a major role in the magnetosphere dynamics (Iijima 2000; Tanaka 2000a, 2003b). A crucial barrier for these considerations has been the difficulty of considering the magnetospheric closure process of FACs. To further relate convection with plasma structure and current systems, the 3D self-consistent structure of the magnetosphere and ionosphere must also be investigated. These limited understandings of the magnetospheric closure process of FAC and uncertainties in its topology must be improved to understand convection; this was in contrast to the fairly advanced studies of ionospheric closure of FAC resulting in geomagnetic variations.

\subsection{Magnetosphere-Ionosphere Convection System}

Much has been done to understand the convection topology by organizing them into cell structures. The flow imposed on the ionosphere under the southward IMF condition consists of twin vortices. In this two-cell convection pattern, antisunward flow of open field lines 
over the polar cap is mapped to the tail lobes and return sunward flow of closed field lines in the auroral zone is mapped to the plasma sheet (Dungey 1961; Cowley 1998; Nishida and Ogino 1998). In the magnetosphere, therefore, this cell is categorized as the merging cell. For the case of northward IMF merging with the southward lobe field poleward of the cusp, Russell (1972) proposed the lobe cell that is confined to open field lines in the tail lobes. In the due-northward IMF case, this lobe cell appears as the low-latitude boundary layer (LLBL) cell that is mapped down in the ionosphere as high-latitude reverse convection cells accompanying sunward flows in the center of the polar cap (Burch et al. 1985). A traditional way to understand these convection patterns had been to map down the solar wind potential through the merging structure to the ionosphere (Crooker 1990). With these concepts, the most basic patterns are derived from the merging structure given by superposing a uniform IMF with the dipole field.

When the IMF $B_{y}$ is nonzero, closed field lines also reconnect with the IMF even under the northward IMF condition. Complex configurations of high-latitude convection due to IMF $B_{y}$ have been given from the statistical analyses of data obtained by polar orbiting satellites (Heppner and Maynard 1987; Weimer 1995), and ground-based IS and HF radars (Ruohoniemi and Greenwald 1996). Characteristic patterns such as overall dominance of dusk cell and persistent rotation of cell orientations to the earlier MLTs show the deviation from the mirror symmetry with respect to IMF $B_{y}$ (Heppner and Maynard 1987; Ruohoniemi and Greenwald 1996; Tanaka 2001). In a traditional view, these complex structures may be related with the relocation of merging site under the non-zero IMF $B_{y}$ condition (Crooker 1990). The MHD results show that the merging cell under northward IMF conditions appears in the ionosphere as a larger round cell surrounding the lobe cells at the center of the polar cap and as a crescent cell in the lower latitude (on the evening side for IMF $B_{y}-$ and on the morning side for IMF $B_{y}+$ ) (Crooker et al. 1998; Tanaka 1999).

The cell structures show that the ionospheric convection potential correlating with IMF $B_{y}$ and $B_{z}$ components is not a mere projection of magnetospheric convection structure controlled by the IMF, but a result of two-way interaction between the magnetospheric and ionospheric convections (Atkinson and Hutchison 1978; Tanaka 2001). The IMF $B_{y}$ effects show the active role of the ionosphere in the convection system (limitation and redistribution of convection) and reveal the nature of convection structure normally hidden under the symmetry of the system to show the two-way interaction nature of M-I convection. Potential saturation is another phenomenon that makes it clear the active ionospheric role in the convection system (Siscoe et al. 2002). When the IMF is very large and southward, the rate of reconnection at the magnetopause is limited by the ionosphere through the feedback of region-1 current system.

Apparently, it is insufficient to understand the convection as the formation of cell structure through the electric field mapping. The mechanical aspect of convection cannot be treated by this concept. For instance, while plasma penetrated from the solar wind tends to accumulate in specific regions such as cusps, the LLBL, the mantle, plasma sheets, and in ring currents, the mechanical processes forming these structures are not directly related with the convection. Traditionally, these magnetospheric plasma structures are understood separately through the equilibrium configuration based on single-particle descriptions or local MHD (Owen and Cowley 1987; Baumjohann et al. 1990a; Cowley 1998). For example, the cusp is understood as the region where magnetosheath plasma flows down along newly opened field lines, and the formation of the plasma sheet is interpreted as the manifestation of pressure balance at field reversal region on the equatorial plane. Similarly, the magnetic field structures are understood in terms of Biot-Savart's law using currents derived from single-particle descriptions, such as magnetization current, polarization current and drift currents. 
The main purpose of this paper is to show that the understanding of convection under the real topologies in magnetic field and current configurations is more essential to clarify the mechanism of disturbance phenomena in the M-I system rather than the investigation of elementary plasma processes, and that the whole of magnetic, current, flow, and plasma structures are organized in a self-consistent way by the convection. The study of magnetospheric convection must consider the plasma dynamics in the limited area bounded by the magnetopause and the ionosphere controlled by the coupling process between the solar wind, magnetosphere and the ionosphere. These interactions between elements depending on topologies that are specific to the magnetospheric and ionospheric regions constitute the compound system. Inside this specified system, magnetopsheric currents, plasma population regimes, FAC, ionospheric current and ionospheric potential must be self-consistently organized into the convection system. Understanding the structure of the convection system is now considered to be an essential element in the comprehension not only of disturbance phenomena such as the substorm (Akasofu 1964; Baker et al. 1996; Lui 1996; Tanaka 2000b), but also of apparently unique phenomena such as the sudden commencement (SC), the flux transfer event (FTE), the traveling convection vortex (TCV), and the theta auroras (Slinker et al. 1999, 2001; Fedder et al. 2002; Keller et al. 2002; Fujita et al. 2003a, 2003b; Tanaka et al. 2003).

\section{Magnetosphere-Ionosphere Coupling Models}

In this paper, the development of magnetospheric structure is considered depending on the MHD approach. In this approach, it is necessary to consider $\mathbf{B v}$ (magnetic field and velocity) as basic parameters to describe the plasma dynamics (Parker 1996, 2000), since MHD does not explicitly include the charge separation process as the cause of electric field. The frozen in principle (1) indicates that the convection electric field is equivalent to the plasma velocity, and never has the cause-effect relationship with the convection velocity. The advantage of this $\mathbf{B v}$ paradigm is that fluid dynamic descriptions using $\mathbf{B v}$ as basic parameters can describe the evolution of convection in the range of possible computation. In contrast to the $\mathbf{B v}$ paradigm of the fluid-dynamic model, the particle modeling assumes that motions of particles generate charge and electric currents, which then induce changes in the electric and magnetic fields that in turn control particle motion. Therefore, EJ (electric field and current) are more basic parameters in this approach (Lui 2000, 2001), and $\mathbf{J}(\mathbf{E})$ becomes the cause of changes in $\mathbf{B}(\mathbf{v})$. Under this approach, collective behavior of plasma can be described without any ad hoc transport coefficients. However, this approach is still too cumbersome to generate global electric field manifesting convection. In other words, EJ paradigm is still too expensive for a real calculation of convection satisfying the requirements of global self-consistency.

When electric field is alternately used as a primary variable in a fluid-dynamic model, the strict consideration of the equation of motion tends to be obscure, turning attention away from mechanics as a force balance. Such confusion arises frequently under the mixed use of Bv and EJ paradigms. For instance, the explanation that the ionospheric convection in the $\mathrm{M}-\mathrm{I}$ coupling system is driven through the projection of magnetospheric electric field onto the ionosphere may seem convincing. Here, a mixed use of $\mathbf{B v}$ and $\mathbf{E J}$ paradigms invites an easy explanation based on an instantaneous propagation image of electric field in an electrostatic model. If the $\mathbf{E J}$ paradigm is pursued to the fullest, a correct explanation must clarify the charge redistribution process due to the particle motion from the evolutionary equations. When the M-I coupling is alternatively explained as the projection of velocity 
from the magnetosphere onto the ionosphere, it becomes natural to consider what forces are acting in the system to produce such projection. In this consideration, the velocity will be mapped down according to the equation of motion (evolutionary equation) together with magnetic stress that accompanies the FAC. Then, (1) automatically determines the electric field from the velocity.

In the Bv paradigm, magnetic fields are carried by the flow as described by the induction equation. In this description, therefore, distortions are created in the magnetic field as it is carried along with the fluid, to induce currents that in turn affect the fluid motion. For any distortions, current is assumed to be available without an upper limit. It is limited only through the suspension of plasma motion caused by a build up of plasma pressure or magnetic force. Therefore, in this paradigm, $\mathbf{J}$ is the result of changes in $\mathbf{B}$. This point is quite different from old geomagnetism in which the main target of study is to replace $\mathbf{B}$ by J. Recently, Vasyliunas (2005a, 2005b) gave a theoretical foundation to understand the Bv paradigm showing that with time scales longer than the inverse of the electron plasma frequency and the special scales larger than the electron inertial length, neither $\mathbf{J}$ nor $\mathbf{E}$ can be calculated directly from evolutionary equations, and alternatively $\mathbf{J}$ is calculated by $\mathbf{B}$ through Ampere's law and $\mathbf{E}$ by $\mathbf{v}$ through the generalized Ohm's law. The advantage of the MHD approach is that with a help of numerical schemes it realizes self-consistent reproduction of convection through the Bv approach. Many new findings were made by this method as will be seen in the following part of this paper (Tanaka 1995, 1999, 2000a, 2000b, 2001, 2003b; Fujita et al. 2003a, 2003b).

The supporters of the EJ paradigm believe that non-ideal MHD processes by themselves generate interesting physics such as FTE, substorm and storm, and that the local non-MHD effects such as current disruption and reconnection overwhelm global self-consistency to unilaterally control magnetospheric disturbances. As a consequence, they state that the EJ paradigm should be selected to examine the physics of the magnetospheric disturbances since they cannot be incorporated into the Bv paradigm. From the viewpoint of this paper, however, global configuration changes that enable instability conditions are more essential for the global structure. For instance, the distortion process of magnetic field by convection controls the progress of the anti-parallel magnetic reconnection that generates global topological changes in the S-M-I system. In addition, a more important requirement for MHD study is that under a local instability condition, another methastable state at a lower energy level must exist as a destination state after the instability. This hidden state must also be realized within the MHD regime (Tanaka 2000b; Sitnov et al. 2000).

\subsection{Global Current System Derived from the Slab Model}

A simple example that aids understanding the principle of the $\mathbf{B v}$ paradigm as applied to the M-I coupling process is the slab model (Fig. 2) (Cravens 1997). Let us consider the slab model as the basis for understanding how the M-I coupling generates convection and current systems. In Fig. 2, M, F, and E represent the magnetosphere (or magnetosheath), ionospheric F layer, and ionospheric E layer, respectively. The entire system is coupled by a magnetic field. The figure represents a moment at which shear motion is generated in the $\mathrm{M}$ region and the front portion is just beginning to move. The slab model is a simple analogy, but it is well suited to understand why the generation of a global current system is accompanied when the velocity (electric field) is projected from the magnetosphere onto the ionosphere. The magnetospheric convection carries the magnetic field according to the frozen-in principle. Even if it starts from no ionospheric convection, growing distortions generated in the magnetic field cause magnetic tension that accelerates the ionosphere. In 
reaction, the magnetosphere is decelerated, and the convective motion is controlled. If we diagnose modeling results through $\mathbf{J}$ derived from $\mathbf{B}$, the obtained magnetic field distortion is expressed by three kinds of current. These currents are field perpendicular current in the magnetosphere, field perpendicular current in the ionosphere and the FAC between them. As a result, FAC, ionospheric current, and magnetospheric current form a closed circuit. The characteristic of the slab model is that it considers the development of convection within the Bv paradigm. In this model, the electric field and current are the result of distortions in the magnetic field, and are not the cause of changes in the electric and magnetic fields.

In the $\mathbf{B v}$ paradigm, $\mathbf{J}$ is an additionally derived parameter from the MHD results. However, the situation is totally altered in the ionospheric E layer where the MHD is not directly applicable; and instead, an electric circuit analogy (EJ paradigm) must be adopted. Therefore, it cannot be avoided to use $\mathbf{J}$ as a primary variable in the calculation. In the ionosphere, the field perpendicular current must be connected with convection electric field through the Ohm's law

$$
\nabla \cdot \Sigma \nabla \phi=J_{\|}
$$

where $\Sigma$ is the ionospheric conductivity tensor and $\phi$ is the ionospheric convection potential. This equation means that the ionosphere dissipates energy associated with the convection $(\mathbf{J} \cdot \mathbf{E}>0)$ even if the convection is steady. This process is equivalently expressed that the convection is maintained against atmospheric friction through the $\mathbf{J} \times \mathbf{B}$ force exerted by the Pedersen current associated with the ionospheric closure of FAC. Without the FAC, ionospheric current associated with the convection electric field in the ionosphere causes charge accumulation to generate secondary electric field that decelerates the convection. Thus, charge must be continuously removed from the ionosphere by the FAC to maintain a steady convection.

As evident from the slab model, the FAC must accompany magnetospheric closure on the other side because currents are non-divergent. From the standpoint of $\mathbf{B v}$ paradigm, there must be a mechanical force in the magnetosphere that counters the $\mathbf{J} \times \mathbf{B}$ force associated with the magnetospheric closure. In this process as well, consideration of the current helps to understand the mechanics of the magnetospheric convection. The work done by the mechanical force against the $\mathbf{J} \times \mathbf{B}$ force must make $\mathbf{J} \cdot \mathbf{E}$ negative. Generally, this action is referred to as the dynamo. Where the meaning of the dynamo is the energy conversion from mechanical energy to electromagnetic energy. In the dynamo region, as a consequence, $\mathbf{v}$ and $\mathbf{J} \times \mathbf{B}$ must be anti-parallel. The mechanical force countering $\mathbf{J} \times \mathbf{B}$ force can be called the dynamo driver. The required mechanical force is dependent not only on the convection velocity $\mathbf{v}$ but also on the ionospheric conductivity $\Sigma$.

In the M-I system, convection must develop under the equality of the two expressions for electric fields defined by (1) and (2). The former is derived from the magnetospheric convection, while the latter represents the ionospheric convection. The real solution is when these two electric fields coincide with each other. The convection process is at the same time the energy conversion system. Seen from the energy-conversion viewpoint, the crucial point is that the M-I current system encompasses ionospheric closure $(\mathbf{J} \cdot \mathbf{E}>0)$ on one side and magnetospheric closure on the other side $(\mathbf{J} \cdot \mathbf{E}<0)$. Therefore, the convection mechanics can equivalently be revealed from the clarification of these current closure and energy conversion processes.

\subsection{Projection of Velocity and the Current System}

Although the slab model is effective to learn the principle of the convection, the real M-I convection system must be understood under more realistic topology. Many attempts have 
been done to construct a more realistic M-I convection together with FAC system. The key point is how to excite shear motion inside the low- $\beta$ region. It was pointed out by Kiverson and Southwood (1991) that compressional motions generated at high altitude in the magnetosphere cannot reach to the ionosphere, whereas shear (or Alfven-like) motions do penetrate the ionosphere accompanying the FAC. Consequently, steady convection must be constructed with shear motion. As shown schematically in Fig. 1, any convection flow in the relatively high- $\beta$ region in the outer magnetosphere carries magnetic field with it to generate magnetic kinks on the boundary with the low- $\beta$ region. This kink immediately propagates toward the ionosphere as Alfven wave to generate FACs. Thus even in a general configuration, magnetospheric convection is inextricably associated with the FACs and dynamo. Due to the incompressible nature of the convection, plasma and magnetic field motions that are distributed from the magnetosphere to the ionosphere must be organized in a circular configuration without a considerable accumulation at all height. It requires the simultaneous consideration of the entire M-I system including the FAC under a proper self-consistency (Tanaka 2003b). As will be shown in the following part of this paper, the convection must associate not only with the FACs and magnetic perturbations but also with the formation of plasma population regimes to cope with the incompressibility of the inner magnetosphere (Tanaka 2003b).

Reconsidering the Dungey's model in terms of the current structure, Atkinson (1978) constructed the line-tying current model for global convection (Fig. 3). Reconnection at the dayside magnetopause generates a reconnection potential, which is simply the M-I convection potential in an electric field context of EJ paradigm. As is shown from the slab model, this convection potential (velocity) mapping along magnetic field lines from the solar wind to the ionosphere must be realized with the region-1 FAC (Vasyliunas 1970). In Atkinson's model, it is the tangential Maxwell stress at the magnetopause that is driving the convection, and the FAC will necessarily close on the magnetopause as near to the voltage generator as global current topology allows. Atkinson (1978) was the first to consider this configuration and proposed that the region-1 FAC driving the convection directly closes just poleward of the cusp (dashed lines in Fig. 3).

In the current configuration of Atkinson (1978), the line-tying current must overlap on the shoulder of the magnetosphere behind the cusp with the pure Chapman-Ferraro current that is connected to the dayside magnetopause. Dashed lines in Fig. 3 show this overlapping part of line-tying current. In Dungey's model, this region must be the dynamo $(\mathbf{J} \cdot \mathbf{E}<0)$ for the region-1 FAC system. Deceleration of magnetosheath flow occurring on this part of linetying current supplies the dynamo energy. However, this configuration results in a peculiar

Fig. 1 Schematic illustration showing the magnetospheric convection coupled with the low $\beta$ region. Convection flow in the relatively high- $\beta$ region in the outer magnetosphere carries magnetic field with it to generate magnetic kinks on the boundary between the low- $\beta$ region. This kink immediately propagates toward the ionosphere as Alfven wave (dashed arrows) to generate FACs (solid arrows). Thus, magnetospheric convection is inextricably associated with the FACs and dynamo

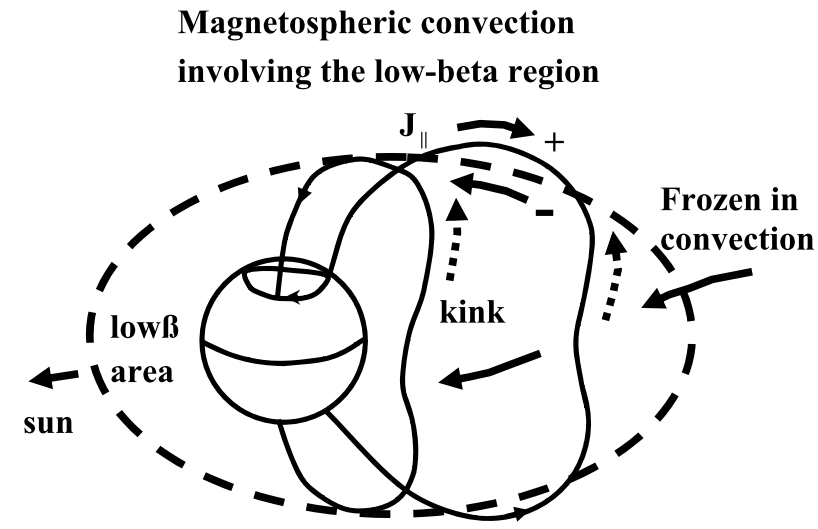


Fig. 2 Slab model of the convection. M, F, and E represent the magnetospheric (or magnetosheath) domain, the ionospheric F layer, and the ionospheric E layer, respectively. The entire system is coupled by a magnetic field (solid lines with downward arrow). The figure represents a moment at which shear motion is generated in the $\mathrm{M}$ region and the front portion is just beginning to move. In this moment, FAC (white arrows), ionospheric current (solid arrows), and magnetospheric current (solid arrows) form a closed circuit. $\mathbf{E}$ and $\mathbf{J}$ are anti-parallel $(\mathbf{J} \cdot \mathbf{E}<0)$ in the magnetosphere, while they are parallel in the ionosphere $(\mathbf{J} \cdot \mathbf{E}>0)$

\section{Slab model of the magnetosphere-ionosphere convection system}

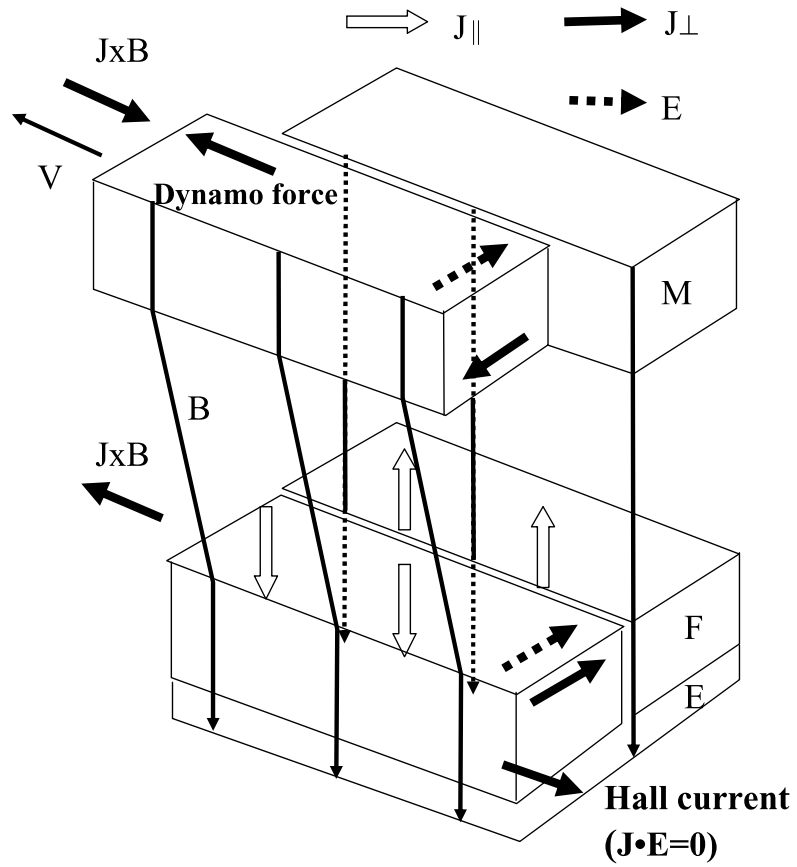

current structure in which the same current simultaneously branches to the pure ChapmanFerraro current and region-1 FAC at the flank magnetopause (Fig. 3). While $\mathbf{J} \cdot \mathbf{E}<0$ is reasonable for the region-1 FAC system, it is not so reasonable for the pure Chapman-Ferraro current. Another unphysical configuration seen here is that magnetopause current becomes stronger on the shoulder part than on the dayside magnetopause against the requirement for stronger magnetopause current in the dayside to cope with large dynamic pressure of dayside solar wind. In the line-tying current model, therefore, the confinement of earth's magnetic field conflicts with the generation of region-1 FAC. This may be a reason for some people to state that the generation of open field lines and following stretching is the primary cause of magnetotail formation (Cowley 1998, 2000). The magnetosphere is confined to a comet-shaped cavity primarily by dynamic pressure, and convection and magnetic topology inside the cavity is controlled by the reconnection structure.

The above concept (Cowley 1998, 2000) is well reflected in popular schematic drawings of the magnetosphere in which magnetic field lines from the polar cap are connected to the IMF at an unusually near-earth point on the magnetopause (for example Stern 1983 and Lyons 1992), although some people drew the polar cap open field lines in a different way so as to be extending inside the lobes as far as the deep tail without an easy connection with the IMF (for example McPherron et al. 1973). Similar interpretation for the popular Dungey's open model was drawn by Stern (1983) from the FAC view. In this view, the region-1 FAC is extended as far as the solar wind region piercing the magnetopause.

Sonnerup (1980) examined an M-I convection model in which velocity is projected onto the ionosphere with the FAC generated by the dynamo in the LLBL. This dynamo is driven through viscous force as proposed by Axford and Hines (1961). The topology of this model 
Fig. 3 Atkinson's line-tying current. In the upper panel, dashed and solid curves show the magnetopause part of line-tying current and pure

Chapman-Ferraro current, respectively. Lower panel shows the whole loop of the line-tying current. Among the current loop shown in the lower panel, only the dynamo region (dashed line) appears in the upper panel on the shoulder of the magnetosphere

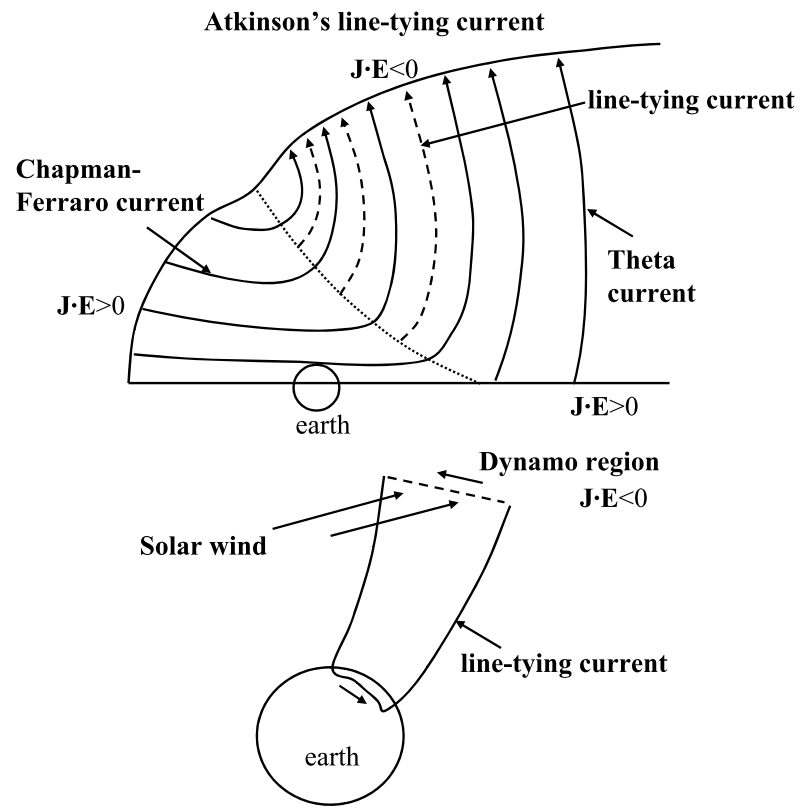

reproduces the actual magnetosphere more strictly than does the slab model, although the configuration of this model cannot treat reconnection driven convection, and thus does not fully reproduce the actual state of the $\mathrm{M}-\mathrm{I}$ convection. This model is, however, very instructive in the sense that it self-consistently includes all element necessary to construct the convection system, such as flows in the magnetosphere and ionosphere, ionospheric current, FACs, and magnetospheric perpendicular current flowing through the dynamo region. The observation that convection reversal occurs in the interior of region-1 FAC (Bythrow et al. 1981) can be interpreted by Sonnerup's model quite well.

The Rice Convection Model (RCM), which treats only the inner magnetosphere, generates the FAC based on the different drift motions of electrons and ions caused by the electric and magnetic fields, and projects the convection potential onto the ionosphere with the FAC (Jaggi and Wolf 1973; Harel et al. 1981). In the ionosphere, the corresponding convection potential and FAC are set to satisfy (2). Electric field thus corrected is mapped back to the magnetosphere to realize the self-consistency in the M-I system. Therefore, the RCM falls into the $\mathbf{E J}$ paradigm. This model reproduces convection in the inner magnetosphere despite the EJ paradigm, because it includes the primary driver of the convection as a boundary condition for $\mathbf{E}$. Global self-consistency in the RCM is still insufficient, because it does not construct a magnetospheric convection including the dynamo process driving the region-1 FAC (Siscoe and Maynard 1991). However, this model was the first that established the advantage of numerical techniques over analytical treatments, and showed the quantitative answer to the question about the shielding mechanism of convection field from the lowlatitude region (Blanc 1978; Tanaka 1981, 1986; Kikuchi et al. 1996). Consequently, the shielding mechanism proposed by Southwood (1977) is numerically realized in this model.

It may not be so difficult to recognize that the plasma structure, current system and convection cannot be independent of each other, since the plasma pressure is indispensable to generate current in each region. To study these linkages between elements including a plausible comparison with real observations, theoretical models must also be constructed under 
a real topology. Plasma regimes must be linked with the convection through the generation process of FAC that requires the formation of the magnetospheric dynamo (Tanaka 1995, 2000a, 2003b). Since the topologies involving these processes have three-dimensional (3-D) nature, the convection and FAC systems must be constructed in a 3-D space having a real topology. The most effective approach that can stand for such study is the MHD simulation; it is at present the only means to reconstruct the 3-D convection system and FAC under the guarantee of global self-consistency.

\subsection{Development of MHD Models}

The above models are insufficient to treat the exact topology of current closure in the M-I system. A crucial method that can overcome this difficulty is the recent S-M-I interaction model by the 3-D MHD simulations coupled with a model ionosphere (2). The configuration and role of the FAC in the reconnection-driven convection system have become gradually apparent through the investigation of MHD simulated convection (Ogino et al. 1986; Tanaka 1994, 1995, 1999, 2000a, 2000b, 2001, 2003a, 2003b; Fedder et al. 1998, 2002; Siscoe et al. 2000, 2002; Gombosi et al. 2000; Ridley et al. 2002). These MHD simulations have reproduced the ionospheric and magnetospheric closure processes of FAC simultaneously (Tanaka 1995), and revealed the relationship between plasma structure, current system, and convection. In these studies, the construction of a realistic FAC system in a 3-D magnetosphere was the first step to investigate the mechanical structure of global convection in the M-I coupling regime (Tanaka 1995).

However, application of MHD simulation had not been so straightforward because the magnetosphere and the ionosphere exhibit large differences in their characteristics. Four major difficulties arise in the numerical calculation of MHD when adapted to the S-M-I coupling simulation. The first difficulty comes from the situation that the respective sizes of the magnetosphere and the ionosphere are extremely different. To overcome this difficulty and to numerically project the convection onto the ionosphere, MHD simulations using unstructured grids are being studied (Tanaka 1995; Fedder et al. 1998; Siscoe et al. 2000; Gombosi et al. 2000).

A wide range in the magnitude of magnetic field causes the second difficulty. The magnitude of the dipole magnetic field is about 30,000 nT in the ionosphere near the earth, while it diminishes rapidly in the magnetosphere to about $10 \mathrm{nT}$. Therefore, the ratio of variable to intrinsic components of the magnetic field becomes extremely small in the ionosphere. In order to avoid this difficulty, the MHD equations are reconstructed by dividing $\mathbf{B}$ as $\mathbf{B}=\mathbf{B}_{0}+\mathbf{B}_{1}$, where $\mathbf{B}_{0}$ and $\mathbf{B}_{1}$ are known intrinsic and unknown variable components, respectively (Tanaka 1994). In the S-M-I interaction problem, $\mathbf{B}_{0}$ is usually taken to be a dipole field.

The third difficulty concerns non-ideal MHD effects and numerical resistivity imbedded in the scheme (Gombosi et al. 2000). The real magnetosphere including many fluctuations and waves is not necessarily dissipationless. In the MHD regime, non-ideal MHD effects are considered through the transport coefficients. Tanaka (2000b) showed that the solution obtained under the assumption that the magnetotail becomes more diffusive as it goes further downtail gives a realistic substorm signature. In almost all MHD models, however, the distribution of transport coefficients is left to the hand of large numerical dissipation. High resolution schemes such as the total variation diminishing (TVD) scheme are required to reduce numerical resistivity. It is also effective to resolve shock and discontinuities which appear commonly in space plasmas (Tanaka 1993, 1998b; Tanaka and Murawski 1997; Tanaka 
and Washimi 1999, 2002). It was shown by Tanaka (1994) that the TVD scheme can be organized even for the reconstructed equations treating $\mathbf{B}_{1}$. By adopting this method, Tanaka (1995) first reproduced the FAC numerically and reconstructed the 3-D structure of the FAC system. This success was the first step to discuss the self-consistent configuration of the M-I convection system. The following part of this paper will mainly focus on the self-consistent picture of convection system obtained from these MHD simulations employing unstructured grids and reconstructed equations. In the presentation of simulation results in this paper, the $x$-axis is pointing toward the sun, the $y$-axis is pointing toward the opposite direction of the earth's orbital motion, and the $z$-axis is pointing toward the north.

One more serious problem in MHD may be whether the role of curvature/gradient drift is properly evaluated in the equation or not. While the curvature/gradient current is included in the equation of motion through the gradient $P$ term, mass transport by curvature/gradient drift is not included. This problem would lead to a limitation for the description of timedependent processes in the inner magnetosphere (Dezeeuw et al. 2004), although little discussion is given in this paper for these processes.

In the MHD simulation, (2) acts as an inner boundary condition. At the inner boundary, the number of fixed variables must coincide with the number of characteristic lines that are directed toward the calculation domain. Equation (2) fixes two variables through the determination of potential. Up to three more variables are fixed in the inner boundary; radial component of the magnetic field, density, and pressure. However, this number depends on the flow direction in the inner boundary (which depends on whether the polar wind case or precipitation case is being considered). If the flow at inner boundary is inward (precipitation case), density and pressure are determined by the projection of variables from the upper region. These conditions must be chosen using the principle of characteristic lines for hyperbolic equations.

In the MHD model, the current $\mathbf{J}$ is simply another representation of $\mathbf{B}$ given by $\operatorname{rot} \mathbf{B}$. It is not the primary variable that is deduced from the equation as an evolutionary variable (Vasyliunas 2005a, 2005b). Alternatively, analysis of $\mathbf{J}$ in the MHD model is effective to understand the force balance in the equation of motion where $\mathbf{J} \times \mathbf{B}$ must balance with other forces. In addition, $\mathbf{J}$ in the MHD model can show important information for the energy transport process between the magnetosphere and the ionosphere. Thus, $\mathbf{J}$ is indispensable for the diagnostics of the M-I coupling system as well as for the construction of ionospheric convection itself. From these viewpoints, the results of MHD simulations are analyzed in the following part of this paper.

\section{Magnetospheric Convection and the Dynamo}

It is clear from the discussions presented hitherto that the convection can be steadily driven provided that the mechanism supplying electromagnetic energy to the ionosphere is incorporated into magnetospheric convection. This process is called as the dynamo by which mechanical energy (kinetic or internal energy) is converted into electromagnetic energy. However, to investigate the magnetospheric dynamo action under the real topology, the closure path of FAC in the actual magnetosphere must first be identified. The MHD model enables this investigation giving the guarantee for the global self-consistency. Then, to understand the energy conversion process, simulation results are further analyzed to investigate how the convection system is developed after the southward turning of the IMF. 


\subsection{Magnetospheric Closure of FAC}

Figure 4 shows an example of calculated current-line structure in the M-I system. The result in the color panels is obtained for northward IMF condition and represents the case of quiet magnetosphere. Color codes on the current lines show the magnetospheric plasma pressure along the current lines. Pressure is normalized by the solar wind pressure ( $\left.P_{\mathrm{sw}}=47 \mathrm{pPa}\right)$. Three current bundles are observable in Fig. 4 showing different color tones. In the order that the pressure of current flowing region is high to low, they are the region-2, region-1, and NBZ current systems. This result indicates that the region-1, region-2 and NBZ FACs close at the higher latitude of cusp, near the inner edge of plasma sheet and in the tail lobe, respectively. Evening side region-1 FAC that starts from the evening ionosphere at first goes forward the dusk flank of the magnetosphere along the magnetic field lines. While the FAC follows along the magnetic field line in the low- $\beta$ region, it does not necessary do so at higher altitudes outside of the low- $\beta$ region. Reaching the high- $\beta$ region, the region- 1 current line deviates from the field lines toward high latitudes and proceeds near the magnetopause. Finally it closes with the region-1 FAC from the morning side at the region tailward of the cusp. As for the region-2 FAC, the closure path obtained from MHD simulation coincides with previous results (Jaggi and Wolf 1973). As is shown by Tanaka (1995, 1998a) and Siscoe et al. (2000), the closure region of region-1 FAC extends more tailward in the case

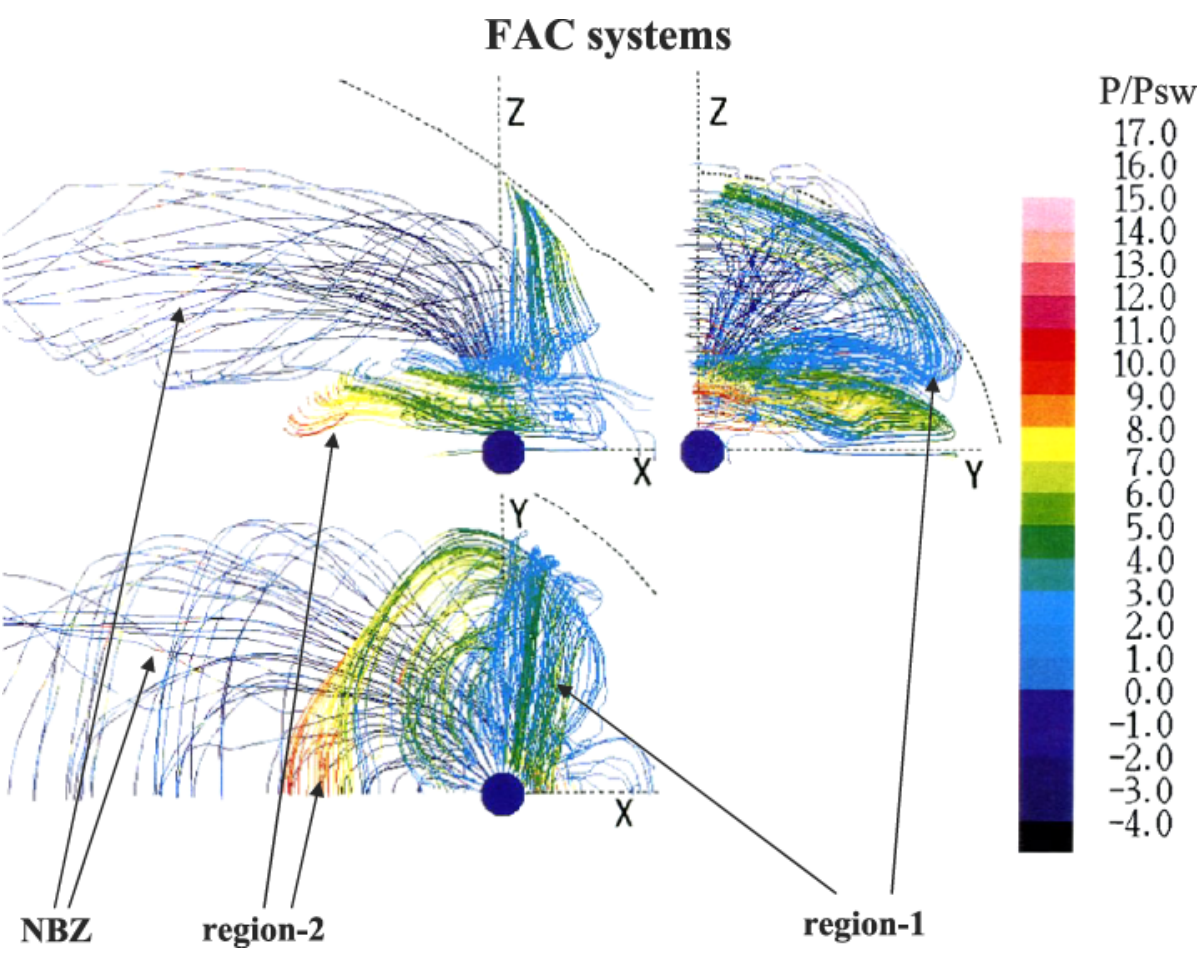

Fig. 4 Global configurations of current lines obtained from MHD simulation, showing region-1, region-2 and NBZ FAC systems. Three panels seen from three directions are shown in this figure. Dashed lines show the position of magnetopause. Color codes on the current lines indicate the magnetospheric plasma pressure along the current lines. Pressure is normalized by the solar wind pressure $\left(P_{\mathrm{sw}}=47 \mathrm{pPa}\right)$ 
Pressure development after the southward turning of IMF
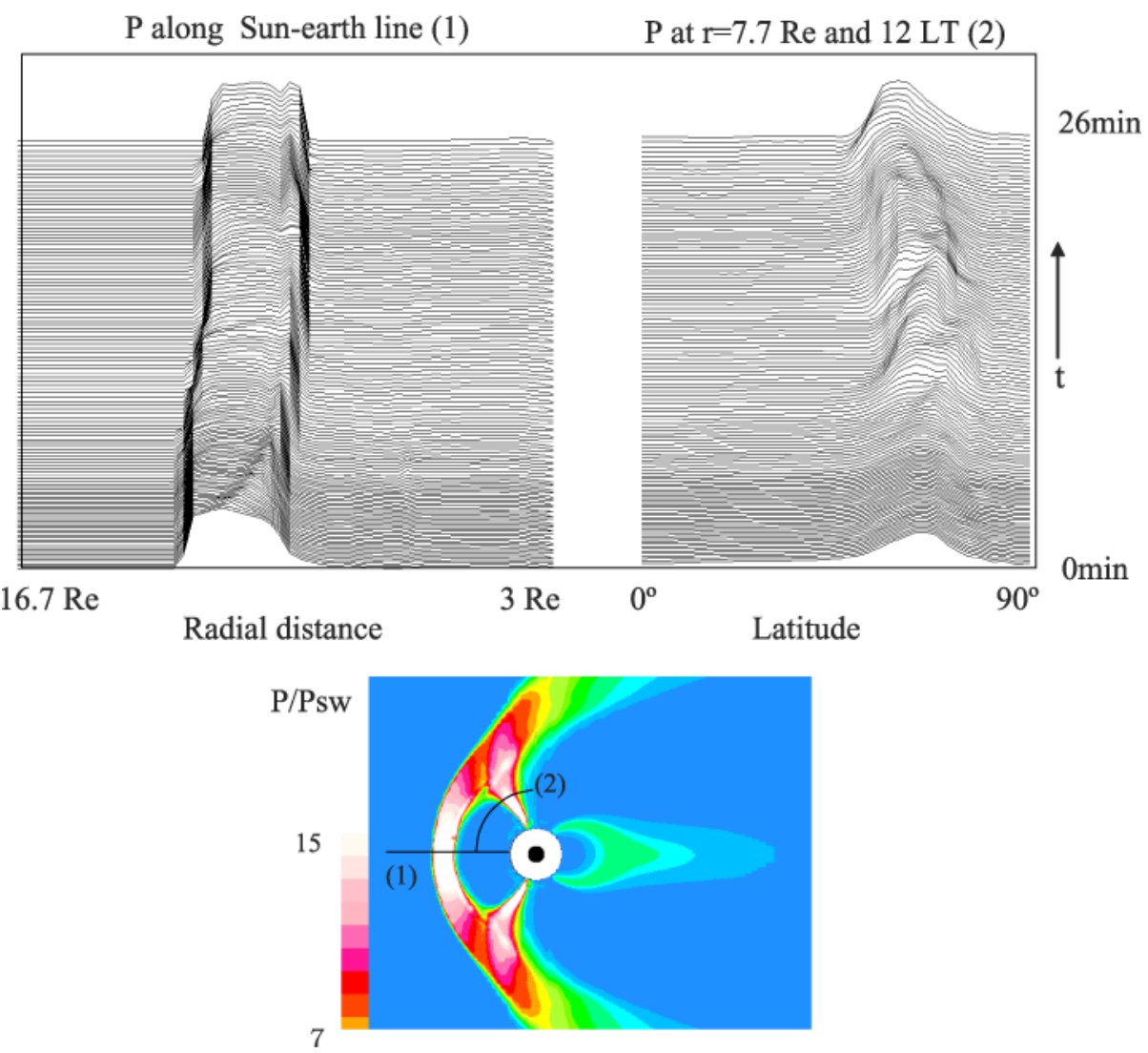

Fig. 5 Pressure perturbations in the dayside magnetosheath (upper left) and in the cusp (upper right) caused by a southward turning of the IMF. Time developments of $P$ distributions in the sun-earth line (upper left) and in the constant latitude line (upper right) are shown during the interval of enhanced convection after southward turning of IMF. The lower inset shows the positions of cutting lines (sun-earth line and constant latitude line) for two data sets. Normalization value $\left(P_{\mathrm{sw}}=47 \mathrm{pPa}\right)$ of color code for pressure $P$ is same to Fig. 4

of southward IMF. The FAC closures must connect the ionospheric current with $\mathbf{J} \cdot \mathbf{E}<0$ regions in the magnetosphere.

Figure 5 shows the time variations of pressure distribution after the southward turning of IMF along the sun-earth line (left panel) and dayside latitudinal line at $r=7.7 \mathrm{Re}$ (right panel). The cut lines for these one-dimensional pressure distributions are shown in the lower inset. In the left panel, the high-pressure region from the bow shock to the magnetopause corresponds to the magnetosheath, while the high-pressure region in the right panel corresponds to the cusp. When the southward IMF reaches the bow shock, a small disturbance appears in the magnetosheath and propagates down toward the magnetopause. However, absolute value of pressure itself is not severely altered. On the contrary, pressure value in the cusp region undergoes a characteristic evolution after the arrival of southward IMF to the magnetopause (right panel). It increases above the magnetosheath level. Pressure tends to increase for 30 minutes. In addition, small perturbations are included in the pressure vari- 


\section{$J \bullet E$, magnetic field lines and convection potential}

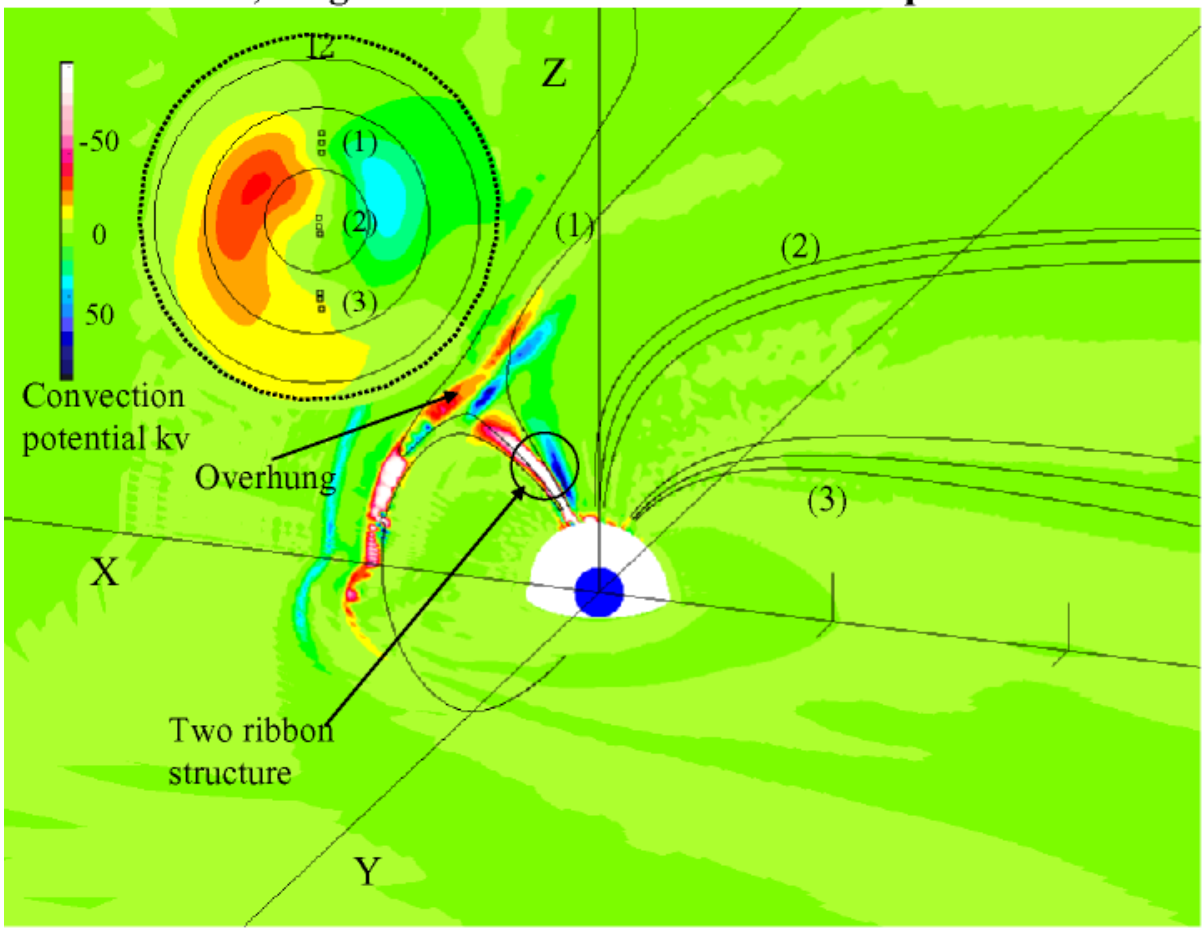

Fig. $6 \mathbf{J} \cdot \mathbf{E}$ distributions (color) in the noon-midnight meridian $(x z)$ and eveningside equatorial $(x y)$ planes, and three groups of magnetic field lines (black lines) that start from the cusp ionosphere $(1)$, the central polar cap (2), and the nightside polar cap (3). Yellow, red, and white show positive $\mathbf{J} \cdot \mathbf{E}$ (pump) in an increasing order, and dark green, blue, and black shows negative $\mathbf{J} \cdot \mathbf{E}$ (dynamo) in an increasing order. Upper inset shows the ionospheric convection potential $(\mathrm{kV})$ by color and footpoints of magnetic field line groups $(1),(2)$ and (3) by dots

ation. These pressure perturbations propagate from low-latitude to high-latitude toward the pole.

What does the cusp-pressure development caused by a southward IMF mean? Figure 6 exhibits a color-coded 3-D display showing the distributions of $\mathbf{J} \cdot \mathbf{E}$ in the noon-midnight meridian and eveningside equatorial planes together with typical magnetic field lines by black lines. These results are plotted under the southward IMF condition. The upper-left inset shows the distribution of ionospheric potential by color and footpoints of typical magnetic field lined by black circles. Three typical groups of field lines in this figure are (1) cusp field line, (2) lobe field lines, and (3) auroral oval field lines. It is noted from the ionospheric potential in this inset that convection is developed to a sufficient extent after the southward turning of the IMF. In Fig. 6, blue color $(\mathbf{J} \cdot \mathbf{E}<0)$ is distributed along the bow shock, indicating that solar wind kinetic energy is converted to the electromagnetic energy through the flow deceleration at the shock. White and red colors $(\mathbf{J} \cdot \mathbf{E}>0)$ in the dayside magnetopause means that magnetic tension caused by a sharp bent of newly reconnected field exerts $\mathbf{J} \times \mathbf{B}$ force on the plasma (Tanaka 2000a).

The most notable feature seen in Fig. 6 is that a pair of red and blue ribbons exists around the cusp, with the red on the low-latitude side and blue on the high-latitude side 
(two ribbon structure). It is seen from Fig. 6 that this region is threaded by typical field lines (1) that are connected to the cusp ionosphere. The blue color also extends towards the mantle. It was already shown by Tanaka (1995, 2000a) and Siscoe et al. (2000)) that this blue region is the magnetospheric closure point of region-1 FAC. However, they did not realize a deep meaning of this region. From this structure, we must aware that even a steady convection without acceleration and deceleration can maintain the dynamo process inside the cusp high-pressure region. On the lower-latitude side of the cusp, convection flow is directed from the low-pressure to the high-pressure sides. Magnetic tension force enables this convection flow against the pressure gradient force (electromagnetic pumping effect). Here the work is done by the electromagnetic force to the plasma, and therefore, this can indeed be considered electromagnetic pumping. On the higher-latitude side of the cusp, this relationship is reversed and the plasma does the work to drive the dynamo. Another notable structure seen in Fig. 6 is the $\mathbf{J} \cdot \mathbf{E}$ overhung structure above the cusp. Positive $\mathbf{J} \cdot \mathbf{E}$ region extending from the low-latitude side of the two ribbon structure distributes near the highlatitude boundary layer (HLBL) so as to cover the top of the cusp high pressure region.

In Fig. 6, the magnetic field lines that pass the regions where $\mathbf{J} \cdot \mathbf{E}$ is intensely colored consist of cusp field lines (1) in both the magnetosphere and the ionosphere. On the contrary, energy conversion is not observable along the magnetic field lines from the central polar cap (2) and the nightside auroral oval (3). These field lines are primarily confined within the tail by the dynamic pressure of fast solar wind flow, rather than formed into the tail configuration by stretching effect of the magnetosheath flow. In the result shown in Fig. 6, therefore, the lobe region is basically force free. On the field lines (2) and (3), the strongest brake for the convection is the ionosphere. The region-1 FAC and the M-I coupling system under the selfconsistent configuration control the convection in the lobe and the polar cap. These results show that the magnetosphere is open at the cusp due to reconnection and that solar wind directly exerts the force at the cusp with energy conversion taking place there. Mechanically the entire magnetosphere is not under a direct control of the solar wind force as described in Dungey (1961), and there is a clear distinction between the cusp and the polar cap.

While the region-1 FAC closes behind the cusp, the region-2 FAC reproduced in the simulation closes at the inner edge of the plasma sheet (Tanaka 1995). This is the topology of partial ring current, and is consistent with the topology predicted in previous studies (Atkinson 1978; Cowley 2000). At the inner edge of the plasma sheet, current is perpendicular to the magnetic field. This perpendicular current is converted into $J_{\|}$in the region where $\nabla P$ is present in the longitudinal direction in the inner magnetosphere; it then flows into (or out of) the ionosphere. The dynamo for the region 2 FAC exists in this region where $\nabla P$ is present in the longitudinal direction. In contrast, $\mathbf{J} \cdot \mathbf{E}$ is positive in the plasma sheet. A FAC system having this structure corresponds to that calculated in the RCM.

\subsection{Energy Conversion by Convection}

Let us re-investigate the force balance in the convection crossing the cusp and the polar cap. It is maintained through the conversion of electromagnetic energy of newly reconnected field lines to the internal energy (pump effect) on the low-latitude side of the cusp and subsequent conversion of internal energy to electromagnetic energy (dynamo effect) on the high-latitude side of the cusp. Therefore, when convection $\mathbf{v}$ is parallel to $\mathbf{J} \times \mathbf{B}$, then work is done by the magnetic field, and pumping occurs with positive $\mathbf{J} \cdot \mathbf{E}$. In contrast, when convection $\mathbf{v}$ is anti-parallel to $\mathbf{J} \times \mathbf{B}$, then work is done on the magnetic field, and the dynamo is realized with negative $\mathbf{J} \cdot \mathbf{E}$ (Tanaka 2000a). 
Whereas kinetic energy prevails in the solar wind, internal energy becomes dominant inside the magnetosphere. In the convection system inside the magnetosphere the kinetic energy is negligible compared with the internal energy and a relation

$$
\mathbf{J} \times \mathbf{B}=\nabla P
$$

is approximately satisfied. Then, the mechanical work $W$ done by the magnetic force $\mathbf{F}=$ $\mathbf{J} \times \mathbf{B}$ in the course of convection is written as

$$
W=\mathbf{F} \cdot d \mathbf{l}=\left(\nabla P+\frac{\rho \mathrm{d} \mathbf{v}}{\mathrm{d} t}\right) \cdot \mathbf{v} \mathrm{d} t \approx \nabla P \cdot \mathbf{v} \mathrm{d} t,
$$

where $d \mathbf{l}$ is the displacement and $\rho$ is the density. The sign of $W$ must be negative to construct the dynamo. For the region-1 FAC, convection works as the electromagnetic-energygeneration system through $\mathbf{v} \mathrm{d} t$ but not through $\rho \mathrm{d} \mathbf{v} / \mathrm{d} t$. As a consequence, dynamo current is carried by pressure gradient current

$$
J_{\perp} \approx-\nabla P \times \frac{\mathbf{B}}{B^{2}} .
$$

As evident from the above discussions, the key feature of the cusp is its role as the energy converter through $\nabla P$. The cusp converts electromagnetic energy to internal energy, and again converts it back to electromagnetic energy, driving the region-1 FAC and consequently the convection. In this view, the cusp is the region where the direct control of solar wind exerts itself inside the magnetosphere to form a strong energy converter inside the magnetopause.

Figure 7 shows schematic drawings of possible dynamo configurations that explain how the dynamo mechanism is related to the cusp formation. In Fig. 7, the upper panel shows the case of flow with acceleration and deceleration, while the lower panel shows the case of steady convection. The dynamo may also be generated by the acceleration and deceleration of convection (Fig. 6, top). In the upper panel, acceleration and deceleration cannot directly be projected onto the ionosphere, since it includes compressional components. In this case, $\mathbf{J} \times \mathbf{B}$ balances with dynamic pressure and a compressional component makes it difficult to realize self-consistent connection with low- $\beta$ shear motion generated by the ionospheric convection.

As can be seen from the bottom panel in Fig. 7, constant-velocity convection can more easily satisfy self-consistency throughout the entire system, because it contains no compressional component and connects smoothly with ionospheric convection. In other words, it is more suited to the M-I convection system in which information can be transmitted only through the shear window. In this case, magnetic tension of newly reconnected magnetic field pumps up cusp high pressure $(\mathbf{J} \cdot \mathbf{E}>0)$ on the low-latitude side and the resulting high pressure generates the region-1 current on the high-latitude side in the course of downward convection $(\mathbf{J} \cdot \mathbf{E}<0)$. Under a restriction of steady convection, therefore, the cusp formation is required for realization of the dynamo process inside the magnetosphere. In this picture, requirement of self-consistency in the convection involves not only the flow (electric field) and FAC but also plasma population regimes in the magnetosphere.

\subsection{Comparison with the Chapman-Ferraro Model and Dungey Model}

Whether the magnetosphere is open (the Dungey model) or closed (the Chapman-Ferraro model) was the basic question in the magnetospheric physics early in the space age. Inevitable consequences of the Chapman-Ferraro model are no convection $(\mathbf{v}=0)$ inside the 
Fig. 7 Schematic illustrations showing the convection and energy conversion process around the cusp. The upper panel shows a configuration that neglects the self-consistency between the magnetospheric and ionospheric convections, while the lower panel shows a configuration that can satisfy the self-consistency between the magnetospheric and ionospheric convections. The lower case automatically explains the generation mechanism of the cusp

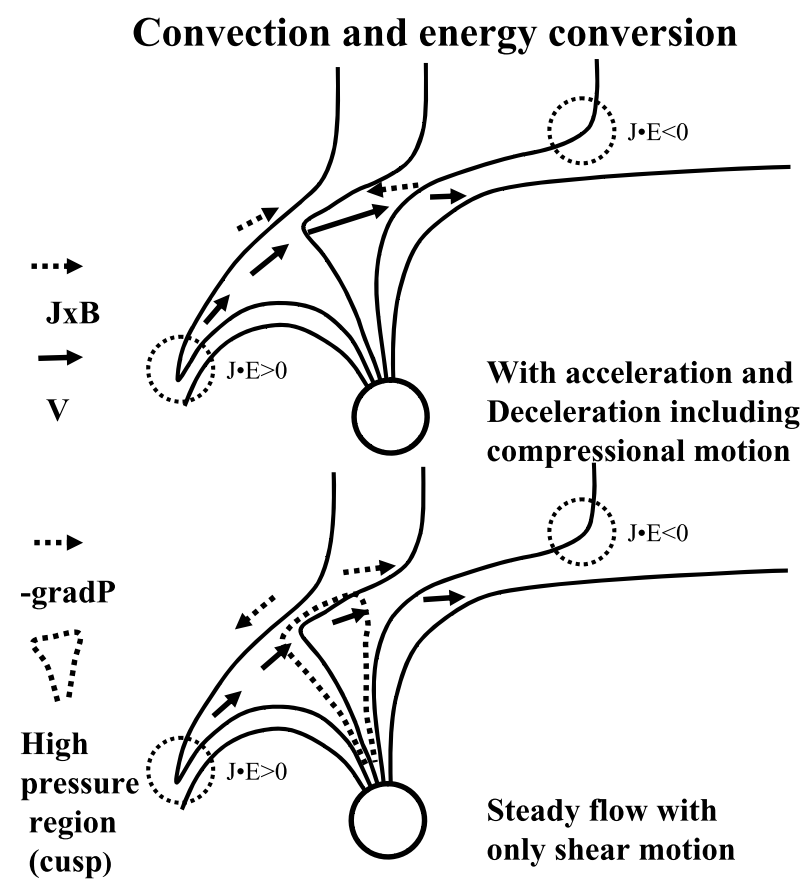

magnetosphere and no energy conversion $(\mathbf{J} \cdot \mathbf{E}=0)$ for the magnetopause current. Since 1961, Dungey's open magnetosphere, which explains convection, has been getting wide support from many researchers. In the traditional open model, tangential Maxwell stress on the magnetopause drives the convection. In the present results, this force extends mainly to the magnetic field lines connected to the cusp. Lobe field lines are nearly force free fields (Fig. 6), although their topology is open. Concerning mechanical structures, therefore, results rather seem to match with the Chapman-Ferraro model. Namely, the stretching of open field lines by magnetosheath flow and their contraction after the tail reconnection seems not to be the direct cause of the convection and tail formation. Confinement by the solar wind dynamic pressure is the primary cause of tail formation, reflecting the fact that flow kinetic energy dominates in the solar wind. In this subsection, interpretations for these inconsistencies between mechanical and topological structures are given by comparing the MHD results with previous models.

Energetically, tangential Maxwell stress on the magnetopause must be the primary driver of the convection as shown in the Dungey's model. Tanaka $(1995,2000 \mathrm{a})$ and Siscoe et al. (2000) showed that a weaker dynamo than that of the cusp dynamo is distributed over a wide area on the magnetopause. In traditional models such as Atkinson (1978), the region-1 FAC system driving the convection is generated from this dynamo as a line-tying current that directly connects the Chapman-Ferraro current and FAC. To consider this configuration is equivalent to stating that the convection is driven directly by the tangential Maxwell stress through the dragging of magnetic field (the Dungey's open model). In the equation of FAC generation, plasma pressure or velocity is indispensable for the conversion between the field-perpendicular current and FAC showing that the FAC cannot be generated in the potential-magnetic-field region (Ogino et al. 1986). Considering no plasma effect inside the magnetopause, therefore, a difficulty arises in the formation of line-tying current that the 
Fig. 8 Configuration of convection system seen from the electromagnetic energy structure. In the text, the order in which energy is transported from the solar wind to the ionosphere is explained according to the numbers shown in this figure. Light and heavy shaded areas represent $\mathbf{J} \cdot \mathbf{E}$ negative and $\mathbf{J} \cdot \mathbf{E}$ positive regions, respectively. Solid lines with arrows show the currents in the magnetosphere. In the $J_{y}$ negative region (2) on the high-latitude side of the cusp, the pointing flux is generated according to (7). Then, the Chapman-Ferraro current transports the energy from region (2) to region (3), where the surface on which the

Chapman-Ferraro current is flowing is not equipotential due to the open magnetosphere (see (6)). The Pointing flux is absorbed in region (3) to generate high-pressure region (4). This plasma energy is converted to the energy of region-1 FAC

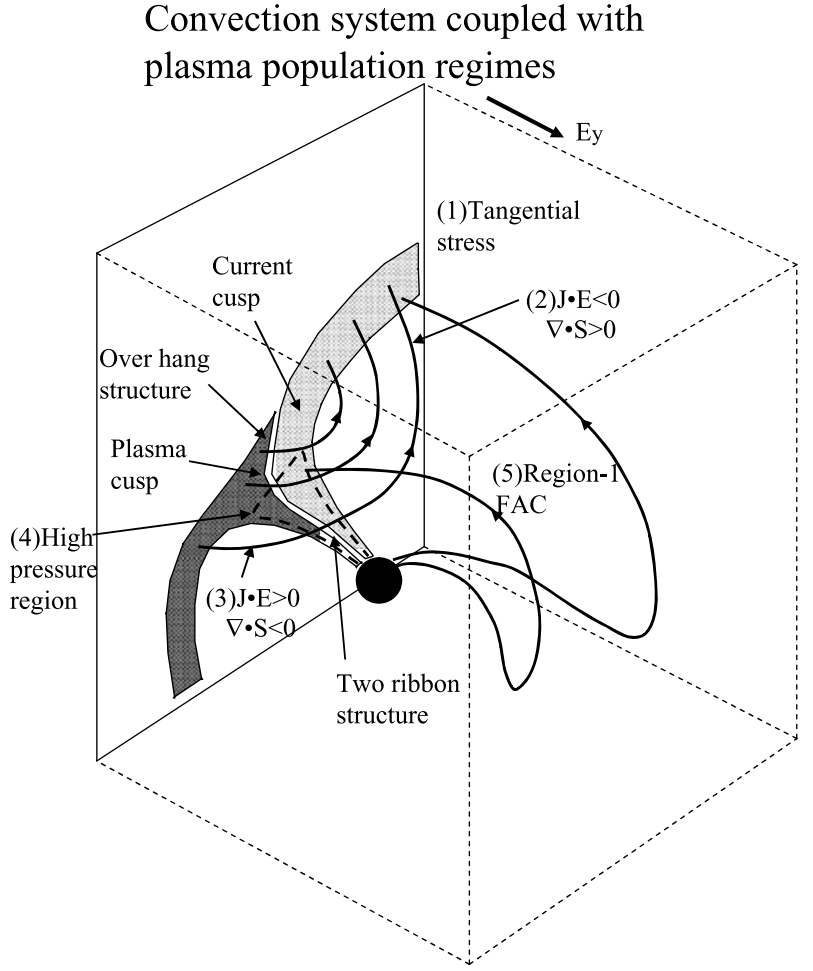

connection between the field-perpendicular current and the FAC must be done outside the magnetopause. This may be one reason for Stern (1983) to have extended the FAC into the solar wind region. In this configuration, a flow deceleration in the magnetosheath will act to form a line-tying current.

Figure 8 summarizes a convection driving mechanism derived from the analyses of MHD results. In the interpretation of this model, the nightside Chapman-Ferraro current is not directly connected with the region-1 FAC even with negative IMF $B_{z}$, but connected with the neutral sheet current in the dayside merging region. It was shown by Tanaka (2000a) that the Chapman-Ferraro current under the southward IMF condition connects negative $\mathbf{J} \cdot \mathbf{E}$ regions on the nightside magnetopause with positive $\mathbf{J} \cdot \mathbf{E}$ regions on the dayside magnetopause. Kink structure of open magnetic field between the tail magnetosphere and the solar wind seen in Fig. 7 indicates that this kink structure on the magnetopause is maintained in the expense of solar wind flow energy to make $\mathbf{J} \cdot \mathbf{E}$ negative. Thus the Chapman-Ferraro current can no longer be work free. The electromagnetic energy generated here is transported to the dayside merging region where $\mathbf{J} \cdot \mathbf{E}$ is positive. This process can be well understood as follows. In the steady state convection where dawn to dusk electric field is approximately written through the potential $\phi=-E_{y} y$, the Pointing flux $\mathbf{S}$ can be written as

$$
\mathbf{S}=\mathbf{E} \times \frac{\mathbf{B}}{\mu_{0}}+\nabla \times\left(\frac{\phi \mathbf{B}}{\mu_{0}}\right)=\phi \mathbf{J}
$$

which has the same divergence as the conventional Poynting flux. In the open magnetosphere, the Chapman-Ferraro current does not flow on an equipotential surface. Then 
the divergence of Pointing flux is

$$
\nabla \cdot \mathbf{S}=\nabla \cdot\left(-E_{y} y \mathbf{J}\right)=-E_{y} J_{y} .
$$

In the $J_{y}$ negative region on the high-latitude side of the cusp, the Poynting flux is generated according to (7) (Fig. 3). Then, the Chapman-Ferraro current transports the energy from the region on the high-latitude side of the cusp to the region on the low-latitude side of the cusp according to (6). The Pointing flux is absorbed in the $J_{y}$ positive region on the low-latitude side of the cusp. As a result, tangential Maxwell stress on the magnetopause acts to increase plasma internal energy around the cusp. The formation of current cusp shown by Siscoe et al. (2000) is another evidence of this configuration. The center of Chapman-Ferraro current ring must shift to high-latitude side of the particle cusp so that the particle cusp is in the positive $\mathbf{J} \cdot \mathbf{E}$ region, because cusp plasma is where the work is done by the ChapmanFerraro current. Overhung structure of positive $\mathbf{J} \cdot \mathbf{E}$ seen in Fig. 6 is another appearance of this mechanism. According to the definition of Atkinson (1978), this current configuration is mechanically same as the tail $\theta$ current system in which tangential Maxwell stress on the tail surface acts to increase plasma internal energy in the plasma sheet through the convection. The plasma internal energy accumulated in the cusp further drives the region-1 FAC. Thus the region-1 FAC system is composed of plasma current and FAC like the partial ring current as defined by Atkinson (1978). Mechanisms to drive the region-1 and region-2 FAC systems are the same two step process; the tangential Maxwell stress on the magnetopause pumps up plasma internal energy inside the magnetosphere, then plasma internal energy drives the FACs.

As shown in Figs. 6, 7, and 8, reconnection is a load and never a dynamo. A load (reconnection) always needs a dynamo to supply energy (Siebert and Siscoe 2002). In Fig. 8, the current system that connects the reconnection and dynamo is the Chapman-Ferraro current itself. It requires a drastic change for the interpretation of Chapman-Ferraro current. In another plausible model proposed by Siebert and Siscoe (2002), the magnetopause reconnection in the subsolar area is threaded by current systems that close through the bow shock. As shown in Fig. 6, bow shock is surely a dynamo which converts the flow kinetic energy to electromagnetic energy. At any rate, clarification of load-dynamo relationship is indispensable to understand a self-consistent convection.

The interpretation adapted in the present model shown in Fig. 8 enables a continuous switch over from the Chapman-Ferraro model to the Dungey model. When IMF $B_{z}=0$, the Chapman-Ferraro current is under the condition of $\mathbf{J} \cdot \mathbf{E}=0$. This state corresponds to the pure confinement of Earth's magnetic field. With decreasing IMF $B_{z}$, the Chapman-Ferraro current behind the cusp becomes to satisfy $\mathbf{J} \cdot \mathbf{E}<0$ and pumps up cusp internal energy through the dayside merging process, not strongly altering the Chapman-Ferraro current topology. The major role of Chapman-Ferraro current is still to confine the Earth's magnetic field, and the lobes are filled with force free polar cap magnetic fields that convect on the ionospheric turn table. This is a modification to the Dungey model so that confinement in the Chapman-Ferraro model continuously changes to convection of plasma and magnetic field for a non-zero IMF $B_{z}$ condition. Multi-layer system of the Chapman-Ferraro current and region- 1 current, one on outside and the other on inside, is a natural consequence of the present model. Indeed, this overlapping configuration was already shown from many numerical simulations (Tanaka 2000a; Siscoe et al. 2000).

\subsection{Coupling between Convection and Plasma Structure}

If the region-1 FAC is replaced by the region-2 FAC and the cusp is replaced by the plasma sheet, the same logic for cusp formation can be adopted to the formation of the plasma sheet. 
In the plasma sheet, earthward $\mathbf{J} \times \mathbf{B}$ force is always balanced with tailward pressure gradient force (Kistler et al. 1992). Convection $\mathbf{v}_{\perp}$ is parallel to $\mathbf{J} \times \mathbf{B}$ and anti-parallel to $-\nabla P$, and electromagnetic energy is constantly being converted into mechanical (internal) energy. As a result, the entire plasma sheet acts like a pump; this structure causes internal energy to accumulate constantly at the inner edge. Plasma convection diverting around the earth to the dayside generates the region-2 FAC converting internal energy to electromagnetic energy. This convection $\mathbf{v}$ is anti-parallel to $\mathbf{J} \times \mathbf{B}$ and parallel to $-\nabla P$. Where is the energy to drive the plasma sheet pump transported from? Clearly, it is from the $\theta$ current system in the magnetospheric tail. The energy driving the $\theta$ current system is exactly the same as that of the region- 1 current, and is generated in the mantle. It can be said that the $\theta$ current system transports the electromagnetic energy generated in the mantle to the plasma sheet.

In the traditional view, the formation of plasma sheet is a manifestation of the requirement for the pressure balance at the field reversal region on the equatorial plane (Baumjohann et al. 1990a; Cowley 1998). This static-balance picture for the plasma sheet must be extended to a more dynamic picture by considering the convection. Although the static balance can surely be realized in an infinitely long tail, the real tail must be attached to the dipolar region toward the earth. In this area, the region-2 FAC system coupled to the convection must participate in the balancing process with sunward $-\nabla P$ force (Tanaka, 1995, 2000a). Since this process involves the ionosphere which dissipates energy, the force balance can no longer be static. As a consequence, the plasma sheet formation and convection become a mutually dependent requirement to maintain the self-consistency in the whole system.

In the magnetosphere physics being discussed today, the generation of plasma regions in the magnetosphere (including the cusps and plasma sheet) is not strictly related with global dynamics such as convection and the dynamo (Cowley 1998). However, in a system in which internal energy is dominant relative to kinetic (motional) energy and the dynamo is driven by internal energy, the magnetospheric plasma regimes and convection become coupled, and plasma structures such as cusps and plasma sheet promote energy conversion (Tanaka 2003b). Without the cusps and plasma sheet, the convection system cannot achieve global self-consistency. The cusps are essential for the generation of region-1 FAC and the plasma sheets for the region-2 FAC.

As stated hitherto, stretching force does not simply excite convection, because it includes an incompressible low- $\beta$ region in the ionosphere. It is inevitable to excite shear motion with the FAC to generate convection. The configuration of shear motion is schematically shown in the left panel of Fig. 9. In this panel, upper and lower vortices represent magnetospheric and ionospheric convections that are equivalent to potential electric field. It is apparent from the configuration of magnetic field that FAC flows at the center of convection vortex. This FAC is equivalent to the downward Poynting flux along the field line which is expressed by $\mathbf{S}=\mathbf{E} \times \Delta \mathbf{B}$ and $\operatorname{rot} \Delta \mathbf{B}=\mathbf{J}_{\|}$. On the contrary, fast mode (middle panel) and slow mode (right panel) motions do not accompany the FAC. Instead, they accompany the change in magnetic configuration $(\partial \mathbf{B} / \partial t \neq 0)$ with a rotational (inductive) electric field. When fast mode and slow mode motions are compared, slow mode motion is characterized by plasma flow along the field line. As shown in this section, shear motion enables an easy coupling to the ionospheric convection. How to power up the dynamo inside the magnetosphere is the main theme of this section and answer is that steady convection flow inside the magnetosphere generates the dynamo by converting internal energy to electromagnetic energy with the help of plasma population regimes.

Figure 10 summarizes how the elements in the convection system are coupled with each other. The motion in the M-I coupling system cannot grow into a large-scale convection without a flow in the low- $\beta$ region including the ionosphere, because the line tying effect of 
Fig. 9 General relationship between velocity and electric field in plasma. The left, middle and right panels are for the shear (rotational, Alfvenic) motion, the fast mode (compressional) motion, and the slow mode (compressional) motion, respectively. Thin lines without arrow show magnetic field lines. Abbreviations are the main impulse (MI), the steady magnetospheric convection (SMC), and the preliminary reverse impulse (PRI)

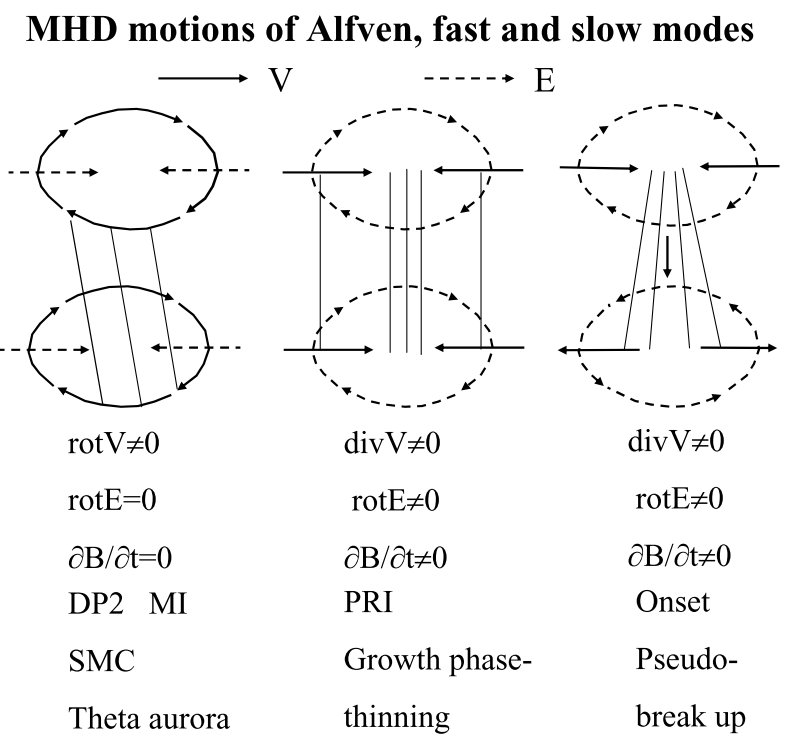

magnetic field that is connected to the ionosphere at rest prevents the global magnetospheric motion (Tanaka 1999). Thus, anti-sunward convection flow that is equivalent to dawn-todusk electric field is mapped down to the polar cap ionosphere through the transmission of stress (Poynting flux) by the FAC (Iijima 2000). This FAC loop must include a dynamo that is powered up by the magnetospheric convection itself. For this power up process, formation of plasma regimes (cusp and plasma sheet) is indispensable, because the convection must be shear (Alfven mode) motion. The loop of steady convection, plasma internal energy, dynamo effect, and FAC is powered by the tangential Maxwell stress. Thus, a steady convection is established in the whole of magnetospheric and ionospheric regions (Stern 1983; Iijima 2000; Cowley 2000; Tanaka 2000a, 2003b).

The convection is really the compound system, since none of elements seen in Fig. 10 can stand alone. In the resulting system, the magnetosphere and the ionosphere recognize their relative motion through the exchange of the FAC. Convection, current systems, and magnetospheric plasma regions are coupled mutually, and cannot be discussed independently. For instance, convection of the substorm growth phase driven by dayside reconnection (Sect. 7), SC convection driven by compression (Sect. 6), and convection at the onset of substorms accompanying dipolarization (Sect. 7) are all generated on this system. Although these individual phenomena have their respective topologies, they share a common principle in the realization of global self-consistency. The magnetospheric physics aims at the clarification of self-consistent structure of S-M-I interaction system. It is the physics of compound system in which an important role of topological effects is pursued as the science of topology.

\section{Ionospheric Closure of FAC and Ionospheric Convection}

The self-consistency in the convection system requires the magnetospheric closure of FAC on one side and the ionospheric closure on the other side. The ionospheric closure process of FAC is a classic problem in geomagnetism, and has been a subject of intense study since Birkeland (Birkeland 1908; Akasofu et al. 1965; Clauer and Kamide 1985; Kamide et al. 
Fig. 10 Schematic illustration of the $\mathrm{M}-\mathrm{I}$ convection system constructed from magnetospheric flow, ionospheric flow, the FAC systems, plasma regimes (pressure), and the dynamo. The upper part shows the requirement for the self-consistency between the elements. This figure summarizes the concept of M-I compound system

\section{Construction of M-I compound system}

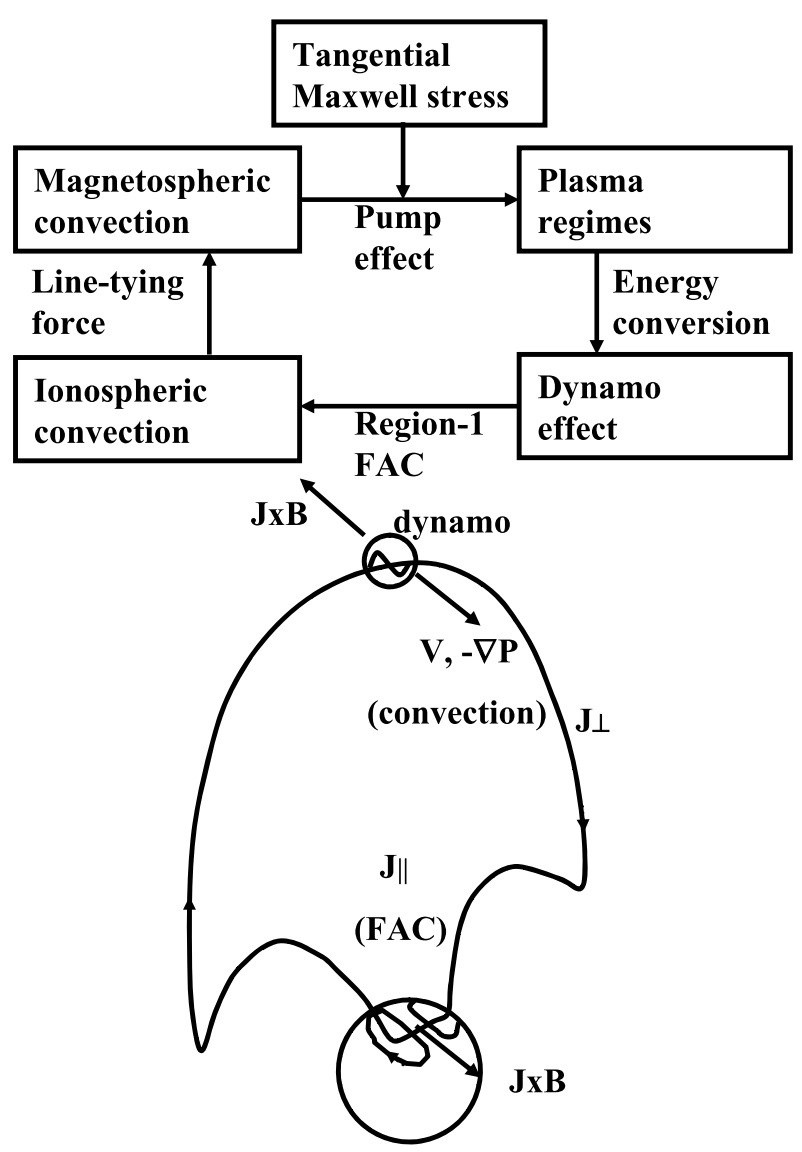

1996; Cowley 2000). However, these researches can be reconsidered from a different aspect by regarding the magnetic variations as the appearance of perturbations in the convection. Since the ionospheric closure is written through the Ohm's law (2), the ionospheric convection can be determined if we can know the two among three elements of FAC $J_{\|}$, conductivity $\Sigma$ and potential $\phi$. Using potential to express the electric field is equivalent to assume that the magnetic field is stationary $(\partial B / \partial t=0)$ in the ionosphere and that the motions in the ionosphere are restricted only to shear motion $(\operatorname{div} \mathbf{v}=0)$. The relationship between $\mathbf{E}$ and $\mathbf{v}$ for this case is illustrated in the left panel in Fig. 9, whereas the case of inductive electric field is shown in the central and right panels in which a stationary magnetic field cannot be maintained due to divergent and convergent motions. Electric potential in the left panel is equivalent to the convection pattern, where ionospheric current produces magnetic field perturbations.

It is well known for a uniform-conductivity case that the steady ionospheric Hall current is perfectly separated from other current systems in the M-I system. Since the Hall current is, in this case, closed inside the ionosphere, it acts no role for the construction of steady current loops in the $\mathrm{M}-\mathrm{I}$ coupling system. Despite this fact, the Hall current is a main cause of magnetic perturbations on the ground because effects of Pedersen current are cancelled out 
by the FAC connected to it (Fukushima 1976). Under the nonuniform-conductivity condition, on the contrary, the Hall current can connect the M-I current system. For instance, the Hall current through the auroral oval can connect the morning and evening region-1 FACs under the non-uniform-conductivity condition. Thus, the convection cannot be studied only in the magnetosphere without considering the development in the ionosphere (Zhang and Carovillano 1993).

Equation (2) coupled with MHD simulations can present three elements simultaneously with a help of an approximate formula to determine the ionospheric conductivity $\Sigma$ from MHD parameters (Tanaka 2000b). Where $\Sigma$ is composed of the solar EUV part and the particle precipitation part. The solar EUV part is calculated as a function of solar zenith angle and the particle precipitation part is calculated as a function of $P$ (simulating the diffuse aurora) and $J_{\|}$(simulating the discrete aurora) that are projected along the field line from the inner boundary. In this system, the MHD process is determined taking (2) as an inner boundary condition to require the self-consistency between electric field and FAC in the magnetosphere as well as in the ionosphere (see Subsect. 2.3). One more advantage of this model is that conductivity distribution also satisfies self-consistency with other elements because conductivity calculated from MHD parameters includes overall consistency with the magnetospheric structure.

\subsection{Ionospheric Convection and FAC}

Figure 11 shows the ionospheric convection in the northern hemisphere calculated for the southward IMF case in which the two-cell structure appears in the polar region. In this figure, the top row shows the case of uniform $\Sigma$ and the bottom row illustrates the case of non-uniform $\Sigma$ caused by EUV ionization and ionization by particle precipitation, while the left column shows results for IMF $B_{y}$ - and the right column illustrates results for IMF $B_{y}+$. In four panels, contours represent $\phi$, and the color codes represent $J_{\|}$. As shown for the slab model, the relationship between convection and FAC in ionospheric closure process can be approximately expressed that the FAC is presented in the center of shear in the ionospheric convection. This situation can be observed quite well from the relationship between the convection potential and FAC presented in the top row of Fig. 11. When $\Sigma$ is uniform, the region-1 FAC approximately corresponds to the shear created by the tailward flow in the polar caps and the sunward flow in the auroral oval, and region-2 FAC approximately corresponds to the shear created between sunward flow in the oval and the slow convection region at lower latitudes (Cowley 2000; Tanaka 2001). In this configuration, the $\mathbf{J} \times \mathbf{B}$ force by dawn-to-dusk Pedersen current connecting dawnside region-1 FAC to duskside region-1 FAC over the polar cap drives anti-sunward flow balanced by neutral drag. However, this picture is apparently oversimplified compared with real observations.

The special point that should be mentioned under uniform conductivity condition is the mirror symmetry between IMF $B_{y}-$ and $B_{y}+$ cases. For IMF $B_{y}-\left(B_{y}+\right)$, there occur deflection of northern cusp flow toward evening $(* 1)(\operatorname{morning}(* 2))$, more-crescent-shaped $\operatorname{dusk}(* 3)(\operatorname{dawn}(* 4))$ cell, more round-shaped dawn $(* 5)(\operatorname{dusk}(* 6))$ cell, and extension of nightside flow reversal on duskside $(* 7)$ (dawnside $(* 8)$ ). Where numbers inside the parentheses with * correspond to the numbers in Fig. 11. Hereafter in this section, we will concentrate our discussion on the ionospheric convection in the northern hemisphere.

Flow deflection in the convection around the cusp toward evening $(* 1)(\operatorname{morning}(* 2))$ is apparently correlated with a well-developed region-1 FAC in the postnoon (prenoon) region. Prenoon region-1 FAC extends toward the high-latitude side of postnoon region-1 FAC for IMF $B_{y}-$, and postnoon region-1 FAC extends toward the high-latitude side of pretnoon 

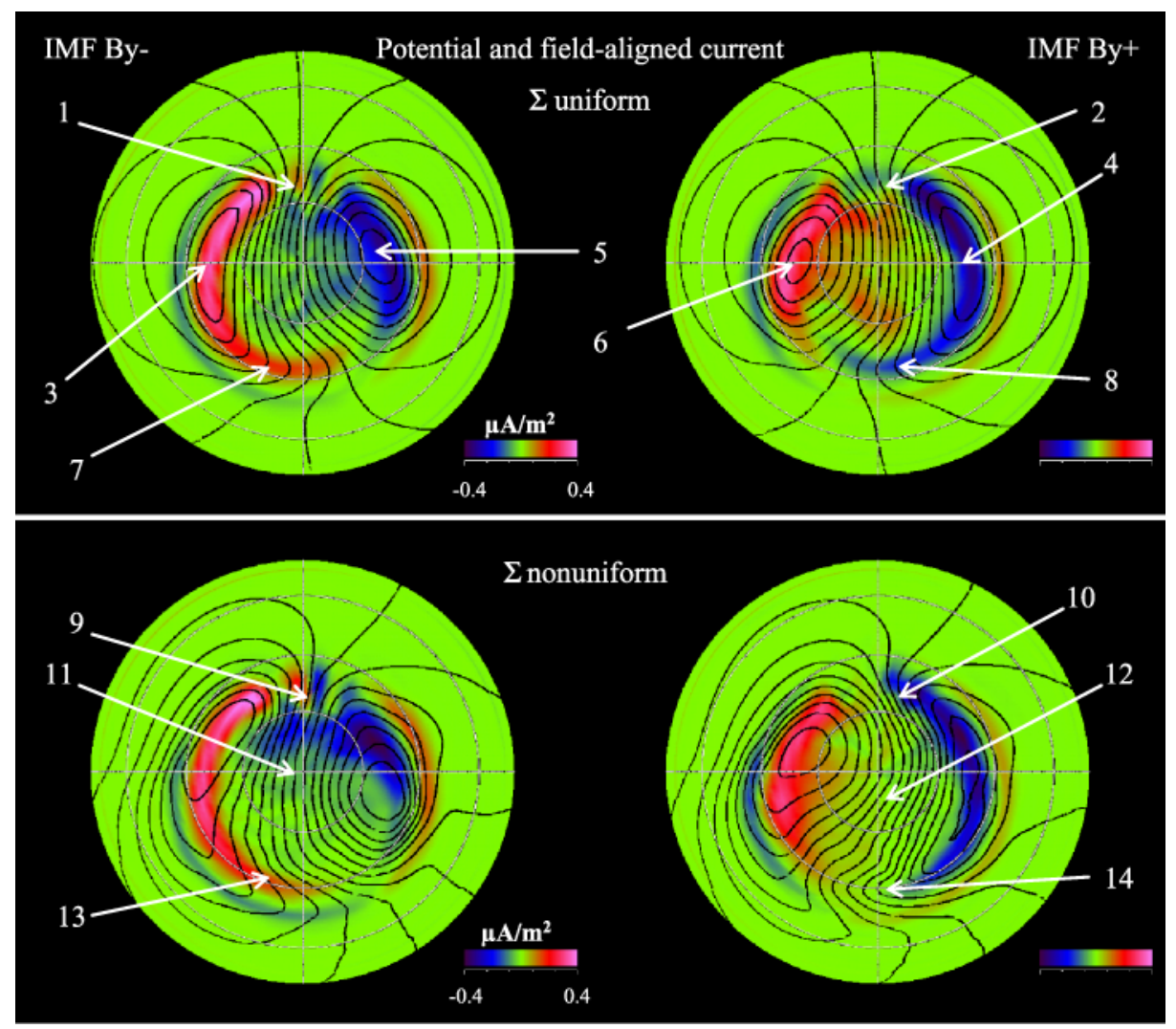

Fig. 11 Ionospheric convection patterns showing the IMF $B_{y}$ and conductivity effects on ionospheric potential and FAC distributions. Potential and FACs are shown by contours and color scales. The left and right columns are for negative and positive IMF $B_{y}$, respectively. The top and bottom rows show the cases of uniform and nonuniform conductivities, respectively. The contour interval is $6 \mathrm{kV}$ and the current unit is $\mu \mathrm{A} / \mathrm{m}^{2}$

region-1 FAC for IMF $B_{y}+$. Whether the cusp FAC feeding DPY ionospheric current is a distinct system or an extension of the region-1 FAC (Friis-Christensen et al. 1985) has been a point of controversy for a long time. In the MHD results, the cusp FAC is an extension of region-1 FAC across noon. The distribution of FAC shows that the magnetospheric driving term is mainly responsible for the IMF $B_{y}$ effect (flow deflection) on the dayside. These IMF $B_{y}$ effects on the convection pattern has been considered as an appearance of the skewed merging process in the S-M-I coupling system (Crooker 1988, 1990).

The equatorward boundary of the dayside cusp is considered to be the projection of reconnection line standing on the dayside magnetopause. It is believed that flow in the ionosphere enters from the auroral oval to the polar cap through this footprint identified as the open/closed boundary. If the exchange of plasma and magnetic flux through the boundary between the auroral zone and polar cap takes place through a narrow opening or gap around the noon, this merging gap becomes throatlike and remaining boundary is a shear reversal. Alternatively, the auroral zone-polar cap boundary is a rotational reversal, if the exchange of plasma and magnetic flux occurs in a broad local time region (Moses et al. 1988). The MHD simulation indicates that the auroral zone-polar cap boundary in the day- 
side is a rotational reversal rather than a shear reversal, over a wide local time range. IMF $B_{y}$ causes the boundary around noon be aligned along the meridian rather than a latitudinal circle and produces an east-west flow component through the boundary, duskward for IMF $B_{y}-$ or dawnward for IMF $B_{y}+$ (Moses et al. 1988; Lockwood et al. 1995). A confirmation is given for the correctness of MHD simulation from these appropriate reproductions of $2 \mathrm{D}$ ionospheric convection patterns.

When $\Sigma$ is non-uniform (bottom row), the relationship between convection shear and FAC is slightly modified, because charge accumulation and the resulting change in electrostatic field occur in the ionosphere in order to maintain current continuity (Atkinson and Hutchison 1978; Tanaka 2001). Compared with the case of uniform conductivity, modifications seen in the bottom row of Fig. 11 appear not only in the potential but also in the FAC. It can be understood from this effect that the projection of velocity (electric field) is not a one-way process from the magnetosphere to the ionosphere but works as a two-way process.

For a realistic conductivity distribution, breakdown of mirror asymmetry is also apparent in cell shaping and position. The most apparent global features for non-uniform conductivity are overall dominance of evening cell and persistent rotation of cell centers to the earlier MLTs. In addition, round-crescent cell pattern is more enhanced for IMF $B_{y}+$. In the cusp region, zone of westward deflection flow for IMF $B_{y}+(* 10)$ is wider than that of eastward flow deflection for IMF $B_{y}-(* 9)$. The flow in the polar cap nearly aligned with moon midnight meridian $(* 11)$ for IMF $B_{y}-$, while the flow in the polar cap has a significant inclination from prenoon to premidnight $(* 12)$ for IMF $B_{y}+$. On the nightside, flow directly deflects toward dawn $(* 13)$ for IMF $B_{y}-$, while westward protruding tongue $(* 14)$ is formed in the potential distribution for IMF $B_{y}+$. As a general trend, these effects of nonuniform ionospheric conductivity seen in the simulated global potential distribution modify the convection pattern so as to match both with the satellite observations (Heppner and Maynard 1987; Weimer 1995) and ground-based observations (Ruohoniemi and Greenwald 1996; Lyons et al. 1997) quite well.

It is only Atkinson and Hutchison (1978) that proposed the mechanism for non-uniform conductivity effects on the convection. They proposed a mechanism for the effect of day night conductivity gradient on the breakdown of the mirror symmetry in convection. Ionospheric conductance produced by solar illumination decreases from the dayside to the nightside across the polar cap. Assume a sharp day-night terminator that crosses the polar cap from 0600 to 1800 LT separating two regions of uniform conductance on either side. The primary electric field is imposed across the polar cap parallel to the dawn-dusk axis. While the primary Pedersen currents flow everywhere along regions of constant conductivity, the primary Hall currents, which flow parallel to the midnight-to-noon direction, are interrupted by the conductivity change at the terminator. In order to satisfy $\operatorname{div} \mathbf{J}=0$ in this circumstance, the electric field is modified so as to include a secondary component which is oriented toward the terminator on both sides of it. Consequently, the antisunward flow in the polar cap is squeezed to the dawnside to break the mirror symmetry with respect to IMF $B_{y}$.

This dawnward squeezing of the antisunward flow makes the dusk cell look like round and the dawn cell crescent-shaped, in the same sense for northern convection under IMF $B_{y}+$. Therefore, the enhancement of round/crescent cell pattern in the northern hemisphere for IMF $B_{y}+$ and the more uniform distribution of flow lines across the polar cap for IMF $B_{y}-$ can be explained from the dawnward squeezing mechanism. It can also explain a wide zone of westward flow that appears for IMF $B_{y}+$ in the dayside around $70-80^{\circ}$ latitude. The rotation of cell centers to earlier MLTs, however, cannot directly be derived from the squeezing mechanism by Atkinson and Hutchison (1978). It is a result of more synthesized effect. 
Combined effects of IMF $B_{y}$ and non-uniform conductivity must be considered more extensively for the modulation of the convection pattern and breaking of the mirror symmetry of the system with respect to IMF $B_{y}$.

Thus, IMF $B_{y}$ effects reveal clearly the electrodynamic structure in the convection system through investigation of the breakdown of mirror symmetry. Results obtained through the $B_{y}$ effects cannot be revealed only from the investigation of due southward IMF case. The fact that violation of the mirror symmetry occurs more severely on the nightside suggests the effects of oval structure for the cell rotation.

Figure 12 shows the comparisons for calculated distributions of the ionospheric conductivity, FAC, and potential. The top row shows the comparison between potential and conductivity, while the bottom row shows the comparison between conductivity and FAC. It can be seen in the top row of Fig. 12 that the auroral zone plays a crucial role for the generation of convection in the nightside ionosphere. For IMF $B_{y}-$, it is obvious that flow lines that extend from the polar cap to the auroral oval along the noon-midnight meridian divert toward dawn when they enter the auroral oval. For IMF $B_{y}+$, on the other hand, the protruding tongue from the morning convection cell extends along the polar cap-auroral oval boundary. As shown above, nightside fine signatures of convection reproduced in the
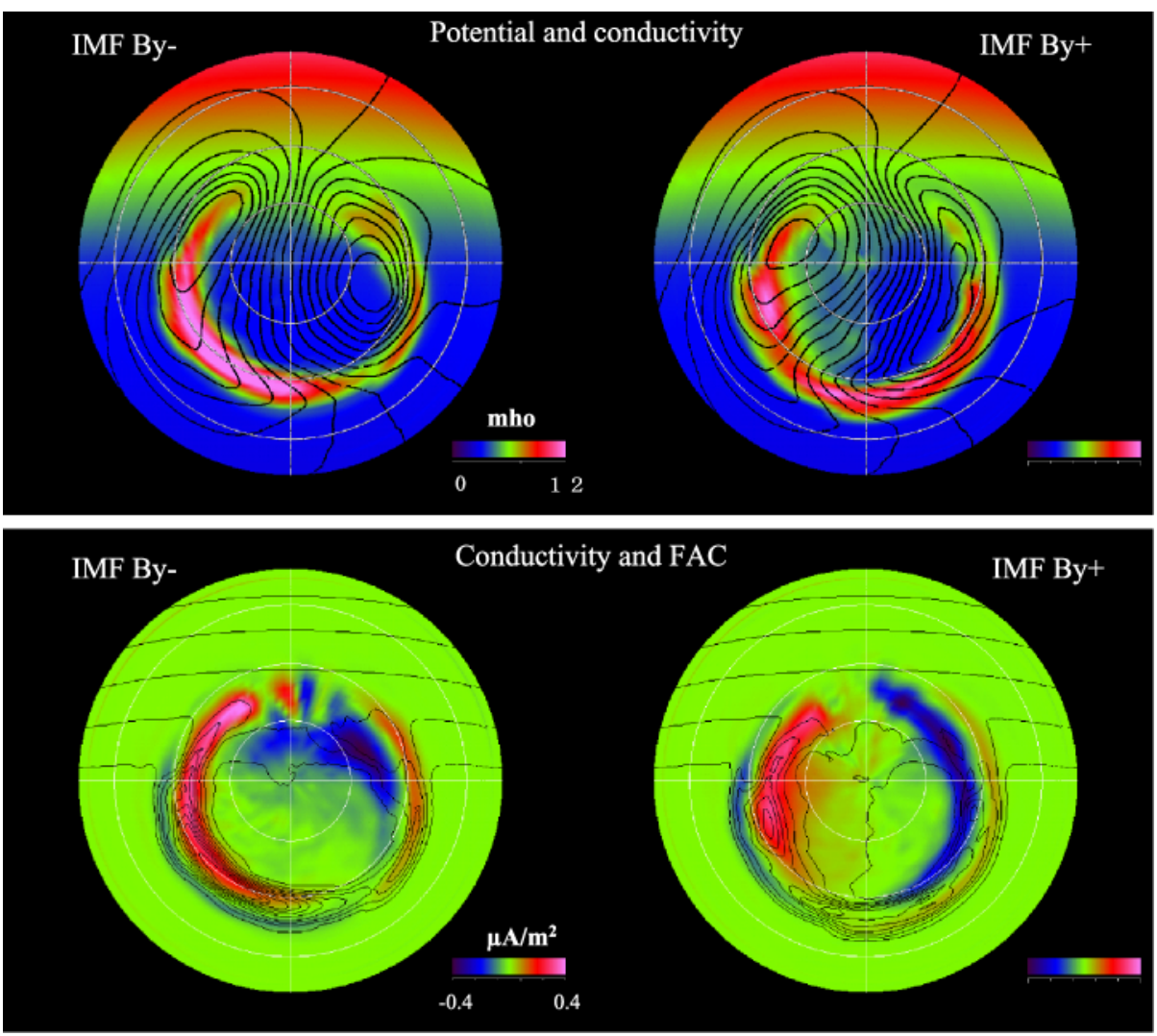

Fig. 12 Relative positions of potential, Pedersen conductivity, and FAC. Electric conductivity and potential are represented by color scales and contours in the top row, and FAC and electric conductivity are shown by color scales and contours in the bottom row, respectively. The unit of color code for conductivity is mho 
MHD simulation give excellent agreement with the satellite observations, particularly with those of Heppner and Maynard (1987). Among them, IMF $B_{y}$ dependent modification of the nightside convection seen in MHD is well explained by considering the Hall current closure process of region-1 FAC, as will be shown in the following subsection.

It is seen from the bottom row of Fig. 12 that the center of the nightside region-1 FAC is situated in the region of steep conductivity gradient on the poleward edge of the auroral oval. This tendency is more apparent in the dusk region than in the dawn region. The relative position of auroral oval and region-1 FAC becomes essential when the connection between the FAC and the Hall current is considered. It can affect the global shaping of the ionospheric convection.

\subsection{Formation of Electrojet}

The most pronounced current system that characterizes the substorm disturbance is known as the DP1 current system (Obayashi and Nishida 1968). The primary characteristic of the DP1 system is a strong intensification of the westward auroral electrojets in a limited region on the nightside. It was pointed out by Vasyliunas (1970) that DP1 current concentration would result from an enhancement of the conductivity in the auroral zone. Thus, it has been the central problem in the area of geomagnetism to construct the 3-D current systems connecting the ionospheric currents and FAC to account for the magnetic perturbation associated with the substorm disturbance (Clauer and Kamide 1985; Kamide et al. 1996). The DP-1 current system begins to enhance in the growth phase and reaches a peek at the expansion phase. In the growth phase, the westward electrojet appears simultaneously with the eastward electrojet in the evening as a pair. In the expansion phase, the enhancement of westward electrojet exceeds that of the eastward electrojet (Kamide et al. 1996). These features in the development of electrojets are controlled by the conductivity enhancement inside the auroral belt. By analyzing the MHD results we can clarify the controlling processes for these features.

Figure 13 shows the divergences of ionospheric Hall (left) and Pedersen (right) currents by color together with their summation by contour, for southward IMF under a realistic ionospheric conductivity condition. In this figure, current divergence (convergence), which can be connected to the downward (upward) FAC, is shown by minus (plus) value. It is a natural consequence expected from the definition to see in Fig. 13 that the summation of two divergence components drawn by the contour reproduces the region- 1 and 2 FACs. On the dayside, the distribution of downward (upward) region-1 FAC coincides with that of the Pedersen divergence (convergence) quite well indicating that the dayside region-1 FAC is mainly connected to the Pedersen current. It is quite natural to observe such situation, because ionospheric conductivity is rather uniform in the dayside region.

In the region extending from pre-midnight to dawn, on the other hand, the distribution of region-1 FAC shows no remarkable coincidence with neither the Hall nor Pedersen divergence. A unique feature seen in this region is that two-band structures having opposite signs extend longitudinally on both sides of the auroral oval. In the band structure, the Hall and Pedersen divergences almost perfectly cancel each other to produce small FAC. This structure shows the southward polarization field with southward Pedersen current in the oval generated through the blocking of northward Hall current. This is a well-known configuration of the Cowling channel, which connects the morning region-1 FAC to the evening region-1 FAC by the Hall current constructing the westward electrojet (Richmond and Kamide 1988). At both ends of the electrojet, it is connected to the FAC. On the dusk side, the upward region-1 FAC must overlap with current convergence (red area). It is apparent in Fig. 13 that 


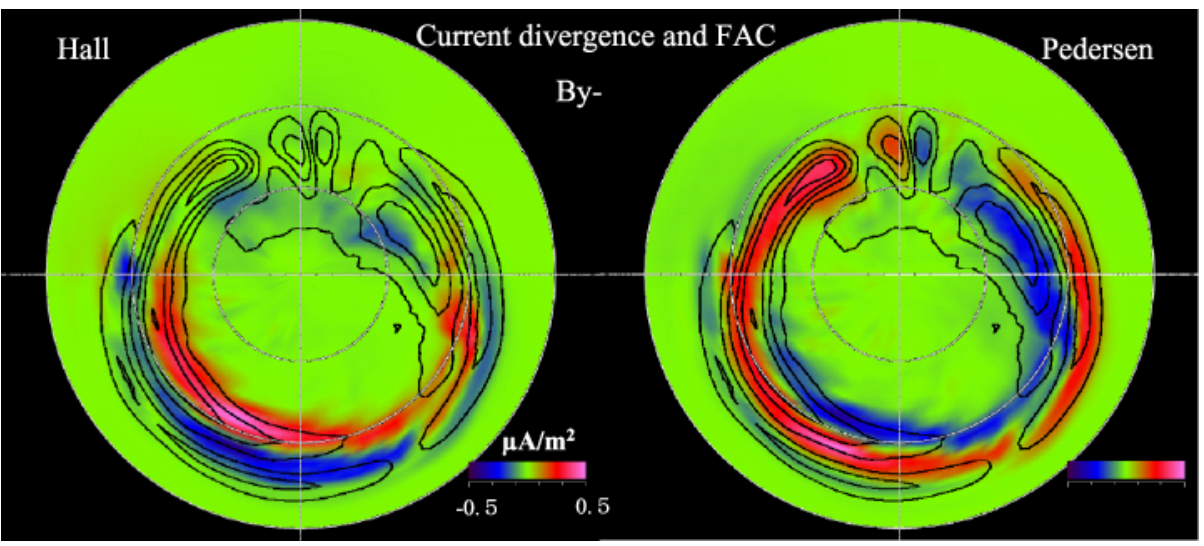

Fig. 13 Divergences in Hall (left) and Pedersen (right) currents shown by color codes. Contours show the FAC (summation of Hall and Pedersen current divergences). The unit of color code for conductivity is mho

in the evening sector the poleward half of the region-1 FAC corresponds to the Hall current convergence while the equatorward half corresponds to the Pedersen current convergence. Even in the morning region, the downward region-1 FAC is connected to the Hall current at the poleward edge. In this region, the region-1 FAC must overlap with current divergence (blue area). A small blue spot of the Hall current divergence is seen around 8 MLT to occupy the poleward side of the dawnside region-1 FAC. As shown in Fig. 6, the Hall current can be directly connected to the FAC under the nonuniform conductivity condition.

Thus the closure process including the Hall current is an additional mechanism for the breakdown of mirror symmetry with respect to the sign of IMF $B_{y}$. Hall current requires southward electric field along the nightside auroral oval regardless of the sign of IMF $B_{y}$, and causes the modification of convection so as to break the mirror symmetry, as seen in the position of cell centers, the inclination of polar cap flow, and the configuration of nightside convection reversals. If perfect mirror symmetry with respect to IMF $B_{y}$ is maintained, the electric field in the midnight auroral oval must be northward for IMF $B_{y}+$ and in this case eastward electrojet must be generated in the auroral oval. This situation (mirror symmetry and eastward electrojet) is prohibited because it is against the formation of the Cowling channel. Thus, we can conclude that the nightside convection is determined so as to maintain the Cowling channel and the current closure process, and consequently mirror symmetry cannot be held on the nightside.

As a consequence, the formation of electrojet is linked to the global shape of the convection field too. In order to establish the Hall current closure process of the region-1 FACs through the auroral oval, it is essential to generate southward electric field in the conduction channel at midnight. To maintain this electric field, in turn, a sufficient number of flow lines must enter the pre-midnight auroral oval and divert toward dawn after entering the auroral oval. Thus, sufficient flow lines must intersect the polar cap-oval boundary in the pre-midnight region. For IMF $B_{y}-$, this requirement is automatically satisfied even if the flow line in the polar cap is directed from noon to midnight, because in the dayside sufficient flow lines are concentrated to the duskside due to the flow deflection toward dusk. For IMF $B_{y}+$, on the other hand, flow lines must be transferred in the polar cap from pre-noon to premidnight in order to maintain sufficient flow lines to the pre-midnight oval. The protruding tongue also acts a role to concentrate flow lines to pre-midnight region. These results indicate that not only the nightside ionospheric convection but also the global shaping of cell 
structure are strongly controlled by the auroral conductivity (Tanaka 2001). It is equivalent to say that the whole convection in the M-I coupling system is controlled and modified to a large extent by the secondary electric field in the ionosphere.

\subsection{Harang Discontinuity}

The ionospheric control of the nightside convection discussed in the previous section is apparently related to a long lasting question about the cause of the Harang discontinuity (Harang 1946; Erickson et al. 1991). On the basis of magnetometer observations, Harang (1946) first found that a discontinuity exists in the auroral current system in the premidnight region. These currents are predominantly Hall current so that the discontinuity delineates reversals of the ionospheric electric field and the convection flow. Thus, poleward of the discontinuity one should observe a southward electric field and eastward plasma flow, and equatorward of the discontinuity a northward electric field and westward plasma flow.

In the case of uniform conductivity shown in the first row of Fig. 11, the dusk (dawn) cell makes a larger contribution to the convection on the midnight meridian for IMF $B_{y}-\left(B_{y}+\right)$, since the convection reversal in the night sector, a distinct feature, can be discerned only in the sector of the crescent-shaped cell. The Harang discontinuity-like structure in the evening (Harang 1946), therefore, appears only for IMF $B_{y}-$ that produces a crescent-shaped cell in the dusk sector. The origin of this Harang discontinuity for IMF $B_{y}-$ is estimated to be in the magnetosphere, because nightside convection reversal is strictly correlated with the region1 FAC extending from dayside. However, its feature does not match with an observational feature that the Harang discontinuity extends slant equatorward with increasing MLT. In the second row of Fig. 11, the configurations of the Harang discontinuity are reproduced both for IMF $B_{y}-$ and IMF $B_{y}+$. Contrary to the first row, a discrepancy between the flow reversal and region-1 FAC is apparent along the Harang discontinuity in the evening. The Harang discontinuity is extending slant equatorward with increasing MLT while the region-1 FAC distributes along constant latitude.

From the satellite observation (Heppner and Maynard 1987; Weimer 1995), the Harang discontinuity in the evening can be seen for both cases of IMF $B_{y}-$ and $B_{y}+$. For IMF $B_{y}-$, the Harang discontinuity is formed through the diversion of flow toward dawn, which occurs on the high-latitude side at about $70^{\circ}$ latitude, and successive diversion toward dusk, which occurs on the low-latitude side at about $65^{\circ}$ latitude. For IMF $B_{y}+$, in addition, a protruding tongue structure of the convection is extending along $70^{\circ}$ latitude from the morning convection cell and helping the generation of the Harang discontinuity. Although the position of tongue structure roughly coincides with that of the morning region-1 FAC, there remains a little discrepancy between them. Consequently, eastward flow on the highlatitude side of the Harang discontinuity is bordered on the poleward side of $70^{\circ}$ latitude by a region of westward flow. Due to this structure, the position of the Harang discontinuity between 2200 and 0030 hours MLT is pushed down to lower latitudes for IMF $B_{y}+$ than for $B_{y}-$. At a fixed latitude near $65^{\circ}$ it implies that the discontinuity will appear in the northern hemisphere roughly one hour earlier for IMF $B_{y}+$ than for IMF $B_{y}-$ (Heppner and Maynard 1987). Rodger et al. (1984) also found the Harang discontinuities in the Northern Hemisphere to be earlier for IMF $B_{y}+$ than for IMF $B_{y}-$. These features coincide with the results in the second row of Fig. 11.

The traditional theories of the Harang discontinuity search its primary cause in the magnetosphere (Erickson et al. 1991). In these theories, magnetospheric convection plays a primary role in the formation of the Harang discontinuity, as shown bellow. The dawnside plasma depletion caused by the curvature/gradient drift in the tail causes cross-tail 
divergence of the cross-tail drift current to require upward field aligned current from the ionosphere. Current closure requires electric fields that are directed toward the center of the upward current. A direct comparison of present result with Erickson et al. (1991) may be difficult, because the present model does not include the mass transport by the curvature/gradient drift which is essential in Erickson et al. (1991). Therefore, the present result may be another possibility for the formation process of Harang discontinuity. In the present model, the dominant role of the ionospheric secondary electric field in the formation of the Harang discontinuity is apparent from the inspection of Fig. 11. This result is deduced from two important features seen in the convection pattern obtained from the simulation. At first, discrepancy between the region-1 FAC and the convection reversal is apparent in the premidnight region in Fig. 11. This result shows that the Harang discontinuity is not directly generated by the magnetospheric driver. Second, the result shown in Fig. 11 indicates that the nightside convection is strongly controlled by the ionospheric conductivity, showing that the secondary electric field generated in the ionosphere plays an important role in the formation of the Harang discontinuity.

While satellite observations by Heppner and Maynard (1987) show the Harang discontinuity in the evening both for IMF $B_{y}-$ and $B_{y}+$, radar observations by Ruohoniemi and Greenwald (1996) observed it only for IMF $B_{y}-$. The reason for this discrepancy is, perhaps, due to the differences in methods of statistical treatment and in the observable area. Heppner and Maynard (1987) estimated the convection patterns from the patternrecognition-based approach. In their statistics, the condition for the IMF is that the IMF remains within a single sector throughout the period 15 and $90 \mathrm{~min}$ in advance. On the other hand, Ruohoniemi and Greenwald (1996) used a bin-averaging approach. In this method, all IMF data were averaged within 12-min intervals. On the nightside, convection data were tagged with the IMF averaged over three previous intervals. It is naturally expected that the pattern-recognition-based approach tends to prevent the smoothing out of fine signatures. In addition, the HF radars pointing toward the polar direction are generally insensitive for the observation of the lower-latitude area.

As mentioned before, the nonuniform-conductivity effect modifies the convection pattern so as to emphasize distinctive features seen in the convection under a uniform conductivity. This effect is remarkable in the crescent/round cell configuration and Harang discontinuity. Although this modification process by the ionosphere is mainly responsible for the Harang discontinuity, the position of the original convection shear caused by the magnetospheric driver itself does not severely differ from that of the Harang discontinuity. Strictly speaking, therefore, the Harang discontinuity is a synthesized effect of several processes in the M-I system, although the most important contribution is from the auroral conductance effect.

\section{Convection Structures under More General Configurations}

Up to here, the driving mechanism of M-I convection system has been discussed mainly assuming due southward IMFs, although distortions of ionospheric convection by IMF $B_{y}$ were investigated in Sect. 4. Generally, simple IMF orientations such as due southward or due northward cases are assumed when simulation results are compared with real observations even for the cases of disturbance phenomena like the substorm. However, such orientation is rarely realized in the real situation. To allow more direct comparisons with observations, it is inevitable to consider cases of IMFs oriented obliquely southward or northward. These general configurations are essential for the diagnostics of compound system 
physics by observations under the real topology. In addition, the magnetospheric configuration under oblique IMF orientations can give essentially unique effects on the dynamical development of M-I system (Tanaka 1999, 2000b; Fedder et al. 2002). What differences are brought about in the convection system under general IMF orientations?

\subsection{Obliquely Southward IMF}

Effects of IMF rotation in the $y-z$ plane (effects of $B_{y}$ ) on the ionospheric convection have been discussed in the previous section. The primary reason for these modulations in the convection must be the $B_{y}$ dependence of dayside reconnection, because the dayside reconnection generating the tangential Maxwell stress on the magnetopause is the first process induced by the IMF after the contact with the magnetosphere (Cowley 1973). There had been two types of structure proposed for the dayside reconnection; local antiparallel merging and global parallel merging (Crooker 1990). Among them, antiparallel merging was predicted by Crooker $(1979,1988)$ from the investigation of superposition model constructed from dipole field and uniform IMF. This concept depends on the expectation that the antiparallel condition must be met somewhere on the magnetopause even between the oblique IMF and dipole geomagnetic field. Later, such antiparallel merging was adopted for the interpretation of results from MHD simulations (Ogino et al. 1986; Tanaka 1999). In this model, the $\mathrm{X}$-type neutral lines converge to the cusps.

Two panels in Fig. 14 show dayside reconnection obtained from the MHD simulation during obliquely southward IMF. The first feature seen in Fig. 14 is a large deformation and a lack of north-south symmetry for the closed magnetic field just before the reconnection. This implies that not only IMF is distorted by draping before the reconnection, but also geomagnetic fields participating in the reconnection are also distorted before merging (Cowley 1973). This is normally referred to as the presence of diffusion region. In the real situation, as shown in Fig. 14, the magnetic configuration in the diffusion region is not so simple as the superposition model by Crooker $(1979,1988)$. If the spatial volume is separated into three parts, the volumes that contain closed field lines, open field lines, and IMFs, the interfacing surfaces between these three volumes are defined as the separatrices on which the diffusion region is spreading (Crooker 1985; Siscoe et al. 2001).

At a proper distance from the subsolar region, the $\mathrm{X}$-type neutral lines are formed outside the cusp and are nearly orthogonal to the IMF direction (Crooker 1979, 1988). In Fig. 14, the antiparallel merging is seen outside the cusp nulls, and the X-type neutral lines outside the cusp nulls converge to the northern and southern cusps. Near the subsolar region, on the contrary, the structure of separator line connecting two cusp nulls is well observable in the left panel in Fig. 14. As pointed out by Crooker $(1979,1985,1988)$, the intersections of separatrices form the separator lines which connect the cusp-type null points. This topology can be well observed from the comparison of left and right panels in Fig. 14. Field lines extending from the northern (southern) cusp form the open-closed boundary in the southern (northern) hemisphere and open-IMF boundary in the northern (southern) hemisphere. The separator line coincides with the intersection of these two surfaces (separatrices). Along the separator line seen in the left panel of Fig. 14, we can find all types of field line, such as distorted IMFs, open field lines and distorted closed field lines. Thus global parallel merging is expected on this line. Reconnection between different types of field lines can occur globally as the result of losing their identity when they run parallel to each other.

As a natural consequence of the separator line converging to the cusp, a complex structure appears in the dayside region. IMFs impinging on the subsolar region between the northern 


\section{Dayside reconnection under the obliquely} southward IMF condition

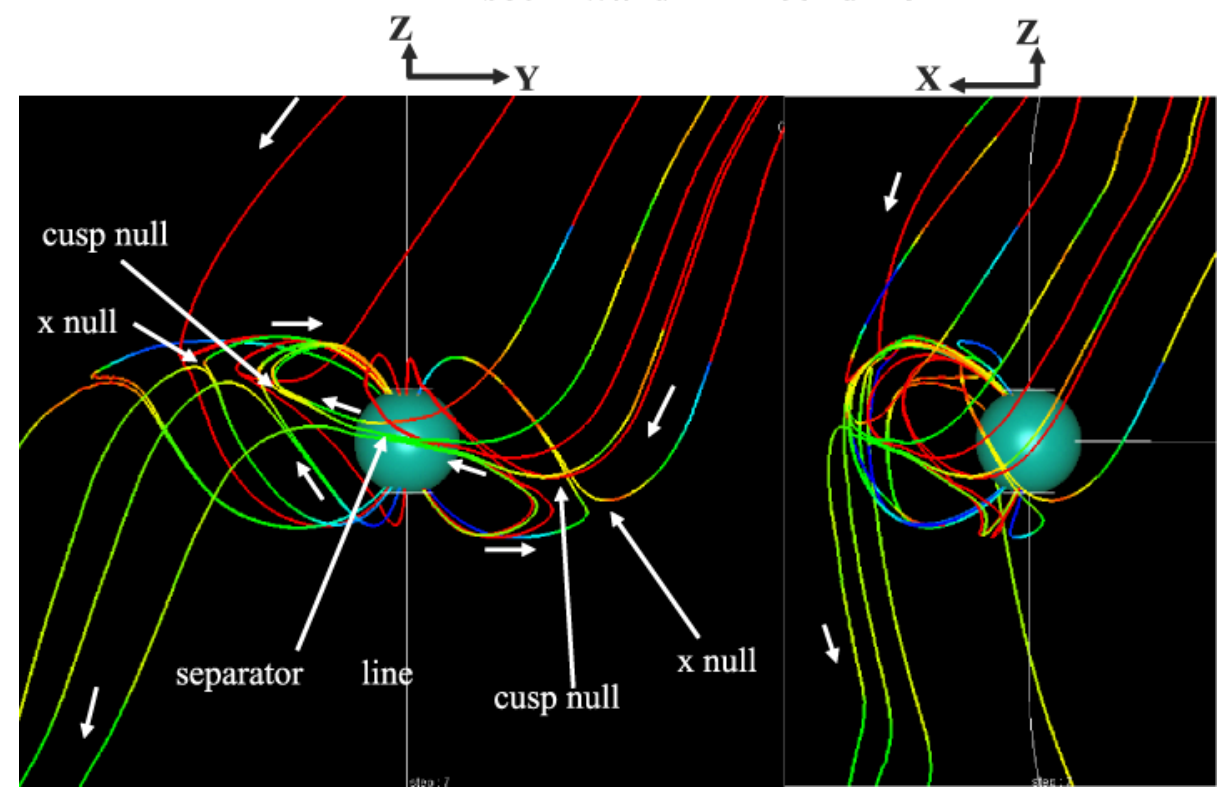

Fig. 14 Configuration of dayside reconnection for oblique southward IMF. The colors are put merely for the identification of each magnetic field line. The left panel is seen from the sun and the right panel is seen from the dusk. The size of green sphere is $3 \mathrm{Re}$. White arrows show the direction of magnetic field lines. These panels show field lines near the surface of open-closed-field boundary (sepatatrix surfaces) which includes $\mathrm{X}$-type neutral lines, separator line and cusp nulls

and southern cusps cannot encounter with the X-type neutral line. While IMFs relatively far from the subsolar region can directly encounter the X-line, IMFs near the subsolar region must suffer severe distortions through the draping process, before global parallel reconnection with field lines extending from the cusps. A lot of IMF field lines pour in on this one dimensional area on the separator line. Since the IMF field lines having different potential gather to one area, their potentials must be adjusted before reaching this area by forming parallel potential (Siscoe et al. 2001). As a consequence, a reconnection concentration zone is produced in the central region sandwiched between the northern and southern cusps, despite that even these IMFs impinging on this reconnection concentration zone must finally be connected to the cusp through the global parallel merging on the separator line. A serious question arising from this structure is whether a steady solution exists or not as the merging configuration. Concerning this problem, Fedder et al. (2002) showed that MHD solution under the obliquely southward IMF is nonstationary even for a steady IMF. The nonstationary magnetic feature occurs in the dayside and evolves as it propagates along the magnetopause to bipolar normal component and enhanced core field. This structure appears recurrently during the simulation. Thus, nonstationary solution in the MHD simulation by Fedder et al. (2002) reproduces observed phenomena called as the flux transfer event (FTE) (Russell and Elphic 1978).

IMFs impinging on the merging concentration zone cannot slip over the magnetopause, because neighboring IMFs having larger collision angles are connected with geomagnetic 
field from the cusp to prevent the crossover slipping between field lines. The MHD simulation by Fedder et al. (2002) indicates that the FTE gives a possible mechanism to resolve flux stagnation in the concentration zone. As a result, dayside reconnection under the nonzero IMF $B_{y}$ condition can be the cause of transient phenomena around the cusp ionosphere (such as magnetopause FTE, the FTE related auroral patch, the dayside auroral transient, and the poleward-moving auroral forms) (Newell and Sibeck 1993; Fasel 1995; Thorolfsson et al. 2000). These progressing ionospheric disturbance events occur during intervals of southward IMF being related to variations of IMF $B_{y}$ component (Stauning et al. 1995). The MHD result shown in Fig. 5 also includes fluctuations like the progressing ionospheric disturbances. In Fig. 5 that shows the development of the cusp pressure after a southward turning of the IMF, poleward moving fluctuations are superposed on the cusp development. However, conventional wisdom prefers a more straightforward interpretation of these phenomena in terms of pulsed reconnection rather than topological effects (Lockwood et al. 1995), without considering that the global configuration of dayside reconnection containing IMF $B_{y}$ may produce a complex and nonsteady magnetospheric structure. Generation of FTE through the global configuration effects shows an example of the fact that magnetospheric physics is the science of topology. Since the convection is incompressible in the ionosphere, the fast ionospheric flow associated with the cusp transient presents a concentrated region of equipotential lines. Even if the pulsed reconnection is assumed, they cannot be directly projected onto the ionosphere. The fast ionospheric flow must be realized either through the generation of a small vortex or, as in Fig. 11, through the bending and concentration of equipotential lines at the cusp. For these models, however, the mechanism of dynamo formation remains unresolved yet. Fluctuation phenomena in the cusp ionosphere ought not to be seen as a strict localized problem. It must be considered inside the framework shown in Fig. 10 connecting to the fact that the cusp is the main driver of magnetospheric convection.

Although IMF $B_{y}$ has a significant effect on the global configuration of dayside reconnection, the resulting FAC system ought to generate mirror symmetry in the convection. By coupling with the ionospheric convection, however, the whole convection loses mirror symmetry (see Sect. 4). Unlike the ionospheric convection, oblique IMF ( $B_{y}$ effect) affects the tail structure little.

\subsection{Obliquely Northward IMF}

The convection structure becomes more complicated for the northward IMF than for the southward IMF. For this case, the global parallel reconnection becomes more dominant than the local antiparallel merging. Many different convection cells are observed for northward IMF both in the magnetosphere and in the ionosphere. Under due-northward IMF condition, two reverse cells appear in the ionosphere with sunward convection at the center of the polar cap, showing that the loal anti-parallel merging does not vanish completely even under the northward IMF condition. In the MHD simulation of $\mathrm{M}-\mathrm{I}$ interaction process, a tadpole-shaped short magnetotail is formed in the magnetosphere corresponding to the reverse cell convection in the ionosphere (Gombosi et al. 2000). In the ideal configuration, the dayside reconnection under due-northward IMF occurs simultaneously in both hemispheres on the higher-latitude side of the cusp. As a result, a closed magnetic field filled with solar wind plasma is formed near noon, together with two detached field lines (isolated from the geomagnetic field) in the tail. In the course of convection, the closed magnetic field is assimilated by the magnetospheric LLBL (Song and Russell 1992). After that, it drifts along the flanks to the tail, and then returns to the reconnection point (Troshichev 1990). Due to 
this structure, a northward IMF generally creates a thick LLBL. From these structures, the magnetospheric counterpart of reverse cell should be called the LLBL cell.

In the case of obliquely northward IMF, the convection structure becomes more complicated. In this case, the lobe cell and merging cell appear in the magnetosphere, and the round cell and crescent cell appears in the ionosphere (Reiff and Burch 1985; Crooker et al. 1998; Tanaka 1999). The lobe and merging cells are named after their physical structures while the crescent and round cells are named after their form of appearance.

The global structure of lobe cell convection under $B_{z}>0$ and $B_{y}<0$ conditions is shown in Fig. 15. This figure shows only open field line convection driven by the reconnection in the northern cusp. There is the southern lobe convection that is driven by the reconnection in the southern cusp and mirror symmetrical to Fig. 15. As shown in this figure, the lobe cell convection is initiated by the dayside reconnection between the IMF and open magnetic field line 4. The dayside reconnection between the IMF and field line 4 in Fig. 15 occurs in the prenoon region to satisfy the antiparallelism. This reconnection results in the generation of open field line 1, which is defined as the type 1 magnetic field line (Tanaka 1999). Together with an open field line 1 (type 1 open field line), the reconnection produces a detached magnetic field line $d$. However, detached field line $d$ is peeled away from the earth and is not a part of the convection. The type 1 open field line remains as an open magnetic field line throughout the convection cycle. It convects to positions 2 and 3 , and finally returns to the original position at 4 . The characteristic of the lobe cell convection is that it is confined to the interior of the lobe and does not include closed field lines. Seen in the $y-z$ plane, type 1 magnetic field lines that form the lobe cell always drift near the magnetopause and do not reach the plasma sheet (Tanaka 1999). As seen from Fig. 15, the footprint of a type 1 magnetic field line drifts in the ionosphere along the central part of the round cell at the center of the polar cap (Crooker et al. 1998). As pointed out by Crooker et al. (1998), drift of magnetic field in the ionosphere from 3 to 4 seems somewhat peculiar, because it drifts sunward while another end in the solar wind drifts anti-sunward. This feeling comes from the concept that the convection is driven directly by the tangential Maxwell stress through the dragging of magnetic field (the classical Dungey's open model). However, it is no longer peculiar if we alternatively consider that the lobe magnetic fields extending from the polar cap are force free and convect on the ionospheric turn table while they are confined to lobe structure by the dynamic pressure and resulting Chapman-Ferraro current (see Sect. 3).

Under the oblique IMF condition, not only open field lines but also closed field lines reconnect with the IMF. The convection cell initiated by the reconnection between the IMF and a closed magnetic field line is called the merging cell. In the northern hemisphere, the dayside reconnection between the IMF $\left(B_{y}<0\right)$ and $\mathrm{C} 1$ in Fig. 16 occurs in the prenoon region to satisfy the antiparallelism. The merging cell convection reaches the plasma sheet, and through a reconnection process in the tail, includes an interval of closed field lines (Crooker et al. 1998). The open magnetic field lines in the merging cell can be classified into type 2 (short draping) and type 3 (long draping) field lines. In the ionosphere, type 2 and 3 field lines have their footprint on the outer round cell and crescent cell, respectively (Crooker et al. 1998). However, the difference between these two types (viewed in terms of magnetospheric convection) results in a somewhat complicated behavior (Tanaka 1999). Especially, type 3 (long draping) structure becomes quite complicated (Watanabe et al. 2004).

Type 2 (short draping) field line shown in Fig. 16 convects at positions 1, 2, 3, and 4 similarly to type 1 . However, at position 4 , the northern type 2 field line becomes a closed magnetic field line $(\mathrm{C} 4)$ through reconnection in the tail with a southern type 3 field line. It then returns to dayside $(\mathrm{C} 1)$ as a closed magnetic field line. It can be seen from Fig. 16 that the footprint of type 2 magnetic field lines drifts along the periphery of the round cell 
Fig. 15 Lobe cell convection for obliquely northward IMF (type 1 magnetic field lines). The lower inset shows the ionospheric footprint of lobe cell by a solid line and other ionospheric convection cells by dashed lines

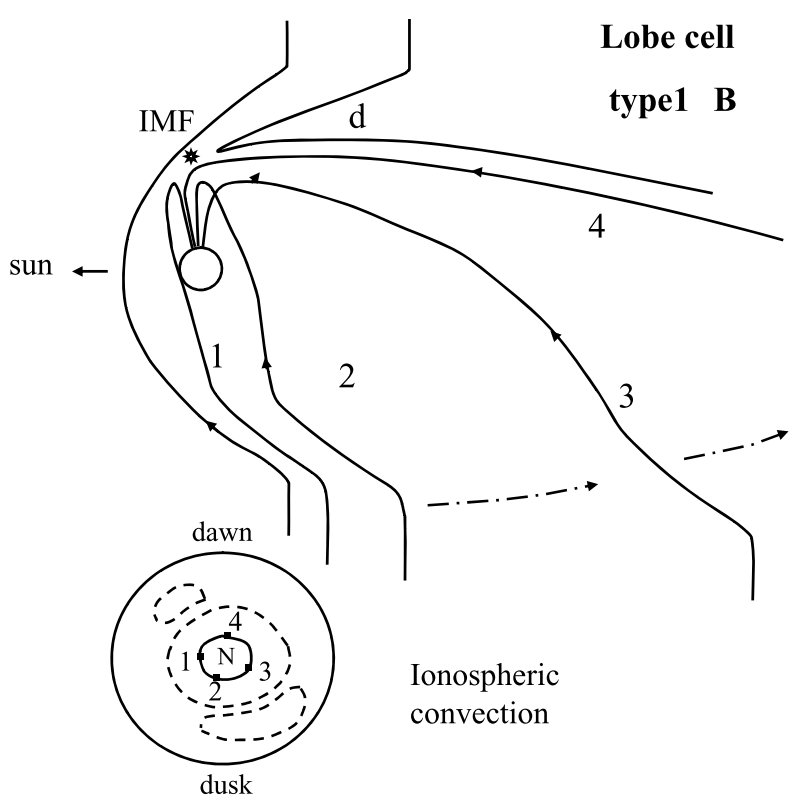

Fig. 16 Merging cell convection for obliquely northward IMF (type 2 magnetic field lines). Open field lines are shown by solid curves and closed field lines are shown by dotted curves. Lower inset shows ionospheric footprint of open field part (solid curve) and closed field part (dotted curve) of the merging cell (type 2)

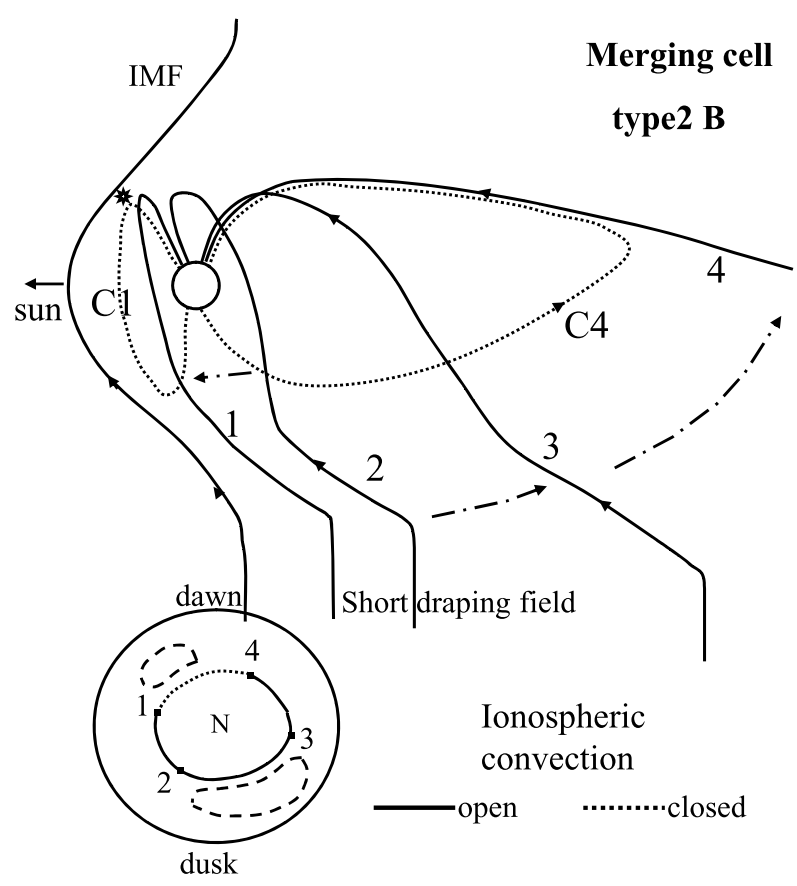

in the ionosphere (Crooker et al. 1998). From the inspection of Fig. 16, it is noticed that generation of a type 2 open field line at position 1 must accompany the formation of open field line of another type (type 3). The type 2 open field line in Fig. 16 generated at position 1 consists of the northern fragment of a closed field line and the southern part of IMF. As a 
counterpart, therefore, another open field line (type 3) that consists of a southern fragment of a closed field line and northern part of IMF must be generated at the same time. The main difference between the type 2 and 3 open field lines is that the former has its footpoint and reconnection point in the same hemisphere (short draping) whereas the latter has them in the opposite hemisphere (long draping) (Tanaka 1999; Watanabe et al. 2004; Vennerstrom et al. 2005). In the ionosphere, type 3 field line convects along the crescent cell. Thus, the crescent cell consists only of the merging cell (type 3 magnetic field line), while the round cell consists of both the lobe cell (type 1 magnetic file line) and the merging cell (type 2 magnetic field line) convections. However, connection of a type 3 field line between the magnetospheric cell and the ionosphere cell is not so straightforward as will be shown in the next subsection.

\subsection{Exchange Cell Convection}

Figure 17 illustrates the development of a type 3 (long draping) field line whose footpoint is in the northern polar cap, and Fig. 18 shows the connection of a type 3 field line between the magnetospheric cell and the ionosphere cell. The type 3 field line shown in Fig. 17 is generated by the reconnection in the southern cusp. It is mirror symmetrical to the type 3 field line generated in the northern cusp simultaneously with the type 2 field line shown in Fig. 16. The type 3 field line in Fig. 17 is shown in Fig. 18 by thick solid and broken lines. In Fig. 18, type 3 field line generated in the northern cusp is also shown by thin solid and broken lines. In this figure, solid and broken lines illustrate nightside and dayside parts respectively.

Dayside reconnection in Fig. 17 between the IMF $\left(B_{y}<0\right)$ and $\mathrm{C} 1$ occurs in the afternoon side of southern cusp. A critical point to be emphasized in this configuration is that the IMF generating this reconnection impinges below (south of) the subsolar point. As a consequence, a field line drifts from position 1 to position 2 toward the tail along the dawn flank (thick broken line in Fig. 18). When the open magnetic field line 1 created by the dayside reconnection arrives at position 2 , it reconnects again with closed field line $\mathrm{C}^{\prime}$ and generates a new open field line 2 and closed field line $\mathrm{C}^{\prime}$. Thus, draping side of open field line changes from dawn to dusk to enable further downtail convection. Along with this switchover process, the footpoint in the northern ionosphere also jumps from $\mathrm{C} 2$ to $\mathrm{C}^{\prime}$. Thus, the convection in the ionosphere from position 1 to 2 can be looked upon as the relay of open magnetic field line from reconnection region at position 1 to the crescent cell at position $2^{\prime}$, producing a third ionospheric cell (in addition to the round and crescent cells). This new convection cell is referred to as the "exchange" cell (Tanaka 1999; Watanabe et al. 2004). In the ordinary consideration, convection cells driven by the reconnection ought to start near noon. However, convection cells located in the nightside are frequently detected from ground observations (Le et al. 2002). At present, the exchange cell is the only mechanism that can explain the ionospheric convection cells confined to the night region.

Seen from the FAC structure, the exchange cell around 9 o'clock (Fig. 17) is equivalent to a 4-layer structure. Whereas Ohtani and Higuchi (2000) explained this 4-layer structure of FAC based on the combination of merging and viscous cells, the exchange cell gives an alternative explanation for the 4-layer FAC structure. In the ionosphere, field lines along the drift path on the round cell from $\mathrm{C} 2$ to $\mathrm{C} 1$ (see southern hemisphere in Fig. 17, also see 4 to 1 of northern hemisphere in Fig. 16) are closed whereas field lines along the drift path on the exchange cell from 1 to 2 (see northern hemisphere in Fig. 17) are open. This situation must accompany a peculiar configuration in which open field line drifting toward the tail exists on the low-latitude while closed field line drifting toward the sun exists on the 
Fig. 17 Merging cell convection for obliquely northward IMF (type 3 magnetic field lines). Upper and lower panels show open field lines and closed field lines included in the convection. Lower and upper insets in the lower panel show footprint of merging cell (for type 3 ) in the northern polar cap and that of counter merging cell (for type 2) in the southern polar cap. Open part of the cell is drawn by solid curves, and closed part is drawn by dotted (nightside) and dashed (dayside) curves
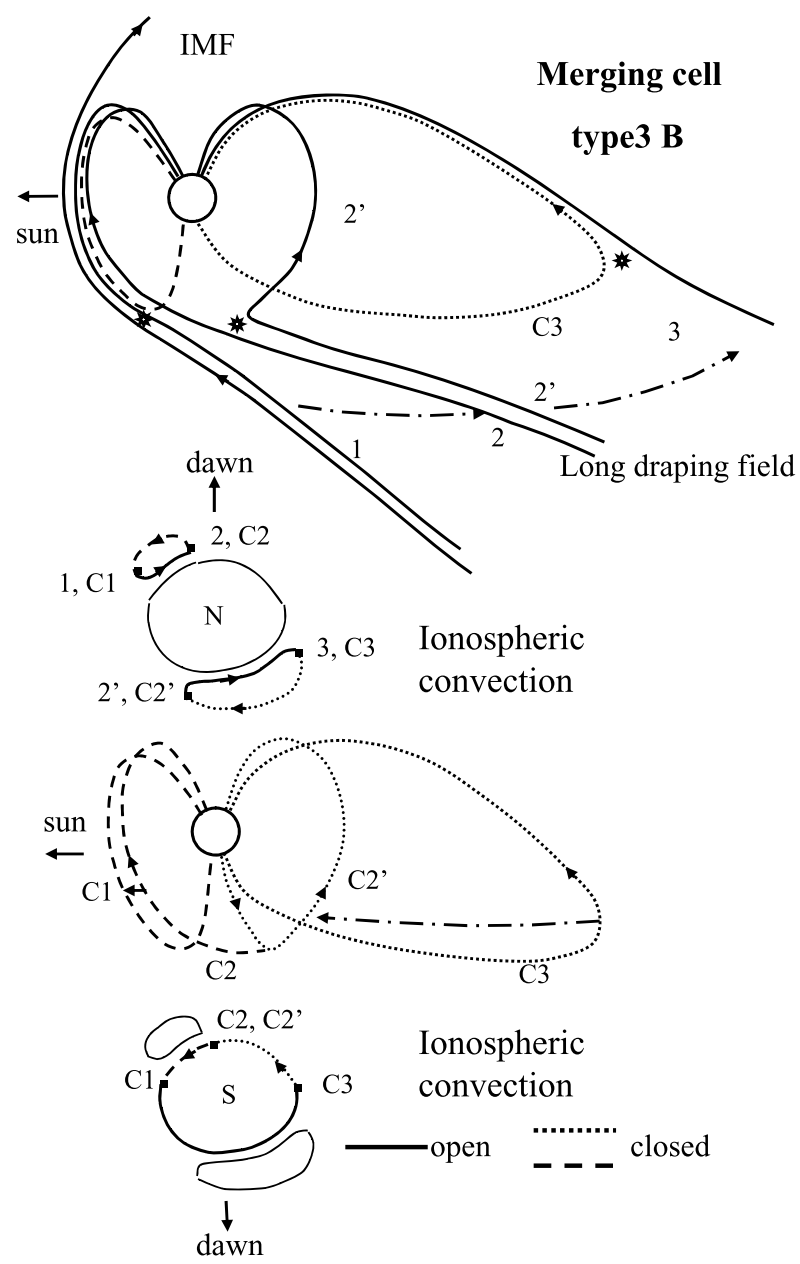

high-latitude side. Apparently this configuration is against the common sense, because in the normal situation tailward open field lines are on the high-latitude side and return closed field lines are on the low-latitude side. Although this structure is quite against the common sense, Watanabe et al. (2004) recently have confirmed this structure from SuperDarn and satellite data, and observationally proved the existence of exchange cell convection.

In the tail region, the type 3 field line moves in the area nearest to the plasma sheet, and reconnects with the type 2 field line in the opposite hemisphere inside the plasma sheet. In the course of this process, the type 3 field line exhibits a strange motion near the plasma sheet. From position $2^{\prime}$ to 3 in Fig. 17, the type 3 field line progresses from the dusk sector to the dawn sector, but it returns to the dusk sector again before the tail reconnection (Tanaka 1999).

From the configurations of type 2 and 3 field lines, it is apparent that a magnetotail with a twisted magnetic structure around the $x$-axis is produced under the obliquely northward IMF condition. The nightside reconnection inside the merging cell must take place in this twisted magnetic-field structure. Since two twisted magnetic fields from northern and southern hemispheres cannot satisfy the antiparallel condition with respect to each other, 
Fig. 18 Cell connection diagram under northward IMF condition with negative IMF $B_{y}$. Dashed and solid curves show the magnetic field lines mainly distribute on the dayside and nightside. Thick and thin curves draw the magnetic field lines reconnected in the southern cusp and northern cusp. Numbers on this figure correspond to that of Fig. 17

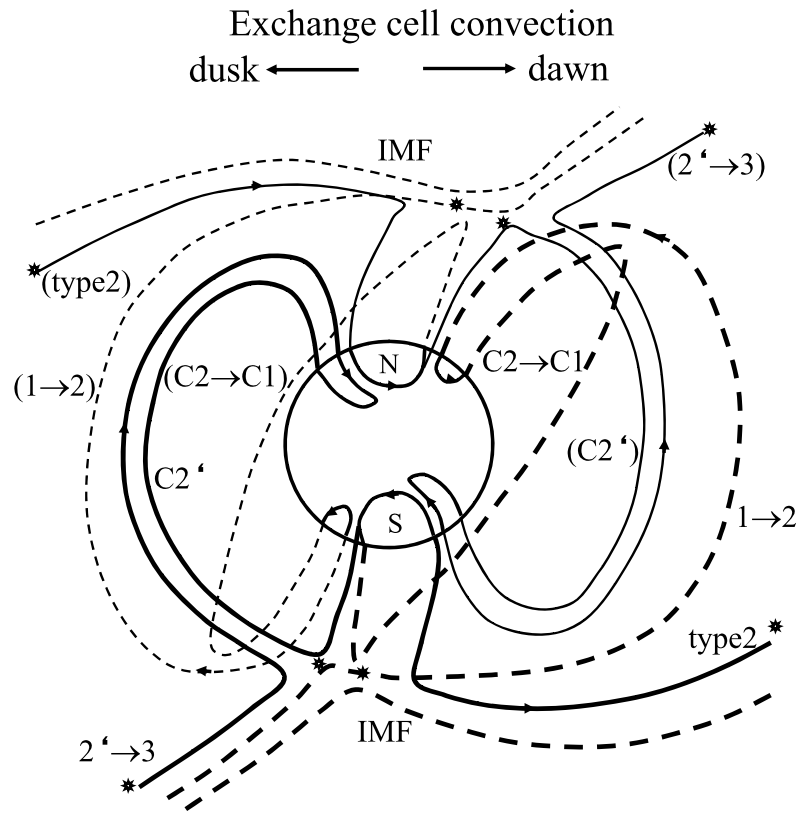

they require an untwisting process in the distant tail before the tail reconnection. This is the cause of strange motion observed for the type 3 magnetic field lines before tail reconnection. This structure can explain the formation of the distant neutral line, and becomes very important as the initial condition for the substorm growth phase (see Sect. 7). More recently, Vennerstrom et al. (2005) gave further confirmations for these IMF $B_{y}$ effects on the merging geometry and convection structure.

The convection flow during a northward IMF, as explained above, is slower than that during a southward IMF. It can be seen from Figs. 15, 16, and 17 that the open field line having a larger $y$-coordinate in the solar wind convects antisunward in the smaller-y-coordinate region in the polar cap. This motion also produces a dawn-to-dusk electric field in the ionosphere. The reason for slow convection is generally understood as that the IMF flux connection to the geomagnetic field through the dayside reconnection is decreased to suppress the penetration of electric field from the solar wind. However, this interpretation is based on the EJ paradigm. It is necessary to explain this convection in terms of the dynamo and FAC, even for a northward IMF. A weak convection under northward IMF should be interpreted as a result from insufficient generation of tangential Maxwell stress on the magnetopause, which leads to smaller internal energy in the cusps (Fig.5).

\section{Behavior of Transient Convections}

It is obvious from both observations and simulations that the convection pattern in the M-I compound system depends not only on the instantaneous values of solar wind parameters but also on the history of how these parameters were changing over some periods of previous time. This fact represents new requirements to study how the $\mathrm{M}-\mathrm{I}$ convection system responses to the external change of solar wind while retaining the self-consistency. However, the more important point to be recognized is that this kind of consideration is the 
basic approach to clarify all types of disturbance phenomena occurring in the M-I system from the viewpoint of compound system. That is to say one can achieve a significant understanding of $\mathrm{M}-\mathrm{I}$ disturbance phenomena by studying them as manifestations of transient response in the convection system for different solar wind conditions (principle of compound system). In this section, we will examine some examples to show that the mechanism of M-I disturbance phenomena can be clarified by considering how the convection system constructed from the incompressible ionosphere and compressible magnetosphere respond most efficiently to the change in solar wind conditions.

\subsection{Reconstruction of Convection after the Southward Turning of IMF}

As shown hitherto, the convection is often explained as being induced by the tailward motion of the magnetospheric plasma due to the tension from IMF anchored to the solar wind, and by back transportation of plasma sunward due to the shortening of magnetic field lines after nightside reconnection events (Cowley 1998). This is widely accepted because, for a long time, the ingenuity ideas on the magnetospheric convection presented by Dungey (1961) led many people to believe them unconditionally. The well-known problem for which such interpretation results in an inconsistent understanding is the reconstruction problem of ionospheric convections after the southward turning of the IMF (Ridley et al. 1997; Khan and Cowley 1999; Murr and Hughes 2001; Hashimoto et al. 2002).

When the IMF switches from northward to southward, ionospheric convection must reconfigure itself from an old state matched to the northward IMF to a new state matched to the southward IMF. Cowley and Lockwood (1992) considered that enhanced dayside reconnection must be projected down to the ionosphere as a fast flow. According to them, fast flow is realized through the progress of two vortices centered at both edges of a merging line that is the ionospheric projection of reconnection region on the dayside magnetopause (see Sect. 4.1). The vortices are small and confined near the cusp soon after the southward turning of IMF but gradually spread to polar cap region according to the progress of convection. It takes more than $30 \mathrm{~min}$ before the vortices grow to the two-cell convection that covers the whole polar cap region. This image of convection reconfiguration is consistent with Dungey's convection in which it takes approximately $30 \mathrm{~min}$ for southward IMF to reach the deep tail.

This concept of gradual reconfiguration is based on the erroneous premise that the nightside ionospheric convection cannot be induced unless the magnetotail connected to the nightside ionosphere knows the change of IMF orientation. However, in the real observation, nightside convection responds immediately to the changes in IMF, as does dayside convection (Kikuchi et al. 1996; Ridley et al. 1997, 1998; Murr and Hughes 2001). In this model, the center of two-cell convection moves little associated with the development of convection. The convection pattern switches instantaneously after the IMF change, while convection strength increases gradually. These results have become more and more convincing according to the improvement of global observation due to the recent extension of radar and magnetometer networks.

Ridley et al. (1998) explained the quick response of nightside ionospheric convection by considering the propagation of fast mode motion. However, as shown in Fig. 9, fast mode motion transmits only rotational electric field, and it does not feed Poynting flux down to the ionosphere. In the consideration given in previous sections, the ionospheric motion is incompressible and the ionospheric electric field can be expressed by potential. Therefore, the motion in the ionosphere is restricted to an eddy (vortex) motion ( $\operatorname{div} \mathbf{v}=0$ ), and the magnetic field is stationary $(\partial B / \partial t=0)$. Based on the principle of compound system, a quick response of the nightside ionosphere must be a quick response of the dynamo process. 
As shown in Fig. 8, the dynamo for the region-1 FAC is situated around the cusp. Since this dynamo area is relatively near the dayside, the whole ionosphere including the nightside ionosphere can respond quickly to the IMF variation. This result at the same time shows that the FAC remains to be FAC only in the low- $\beta$ region at low-altitudes. In the high- $\beta$ region at high-altitudes, it becomes immediately nonparallel to the magnetic field. If the FAC flows along the magnetic field as far as the deep tail, the dynamo must also be in the deep tail. In this case, quick response of ionospheric convection is no longer expected. From the cusp response in Fig. 5, it takes about 30 min to build up the cusp pressure. This time scale just matches with the interval required to increase the strength of convection. Thus, features of ionospheric reconfiguration reflect the principle of $\mathrm{M}-\mathrm{I}$ compound system quite well. This characteristic of non-steady convection also becomes evident in the substorm growth phase, as will be explained afterward.

Observational determination of global magnetospheric configuration is the most difficult problem due to the one point nature of satellite observations. It is difficult even with careful statistical treatments considering various conditions that control the problem. However, as shown above, some evidences for the compound system are seen by comparing the model expectations with observations of 2-D ionospheric convection. They would provide rather better information than magnetospheric observations by satellites to see the nature of convection system. If ionospheric convection is a mere projection of the magnetospheric convection, the whole convection system is no longer a compound system (Siscoe and Maynard 1991). Therefore, for convection as a compound system, the ionospheric convection must be an equal-weighted counterpart of the magnetospheric convection.

\subsection{SC as a Transient Convection Following the Magnetospheric Compression}

A step-like pressure increase and a shock in the solar wind generate a geomagnetic sudden commencement (SC). To consider the SC as a compression of the magnetosphere accompanying an increase in Chapman-Ferraro current may be the most preliminary understanding of this phenomenon. In this understanding, positive deflection of geomagnetic $H$ component worldwidely observed on the ground is a manifestation of increased Chapman-Ferraro current through the Biot-Savart's law. In the more formal investigation of SC, however, it must be studied in a context presented in Fig. 10 (Fujita et al. 2003a, 2003b).

The magnetosphere must achieve a new state of equilibrium to incorporate the stronger dynamic pressure. In the M-I compound system, this process must be a nonstationary response of the convection to a sudden and strong increase of solar-wind dynamic pressure. As we have repeatedly stressed in previous sections, these variations in the M-I system must be regarded as a nonstationary convection. The pattern of geomagnetic field variation during a typical SC consists of the preliminary impulse (PI) and the main impulse (MI). In the high-latitude region, the $\mathrm{H}$-component of ground magnetic field shows a negative (positive) deflection in the PI phase and a positive (negative) deflection in the MI phase in the post-noon (pre-noon) side (Araki 1977, 1994). Among negative and positive PIs, post-noon negative deflection of $\mathrm{H}$ component is called the preliminary reverse impulse (PRI). While the PRI disappears in the mid-latitudes, it appears again in the equatorial region (Kikuchi 1986).

Signatures of magnetic field variations on ground during the SC can be interpreted by a pair of upward and downward FAC, with downward (upward) FAC in the post-noon (prenoon) side in the PI phase, and upward (downward) FAC in the post-noon (pre-noon) side in the MI phase (Araki 1994). This model explains the ionospheric signatures of SC from the mechanism that downward (upward) FAC induces counterclockwise (clockwise) plasma 


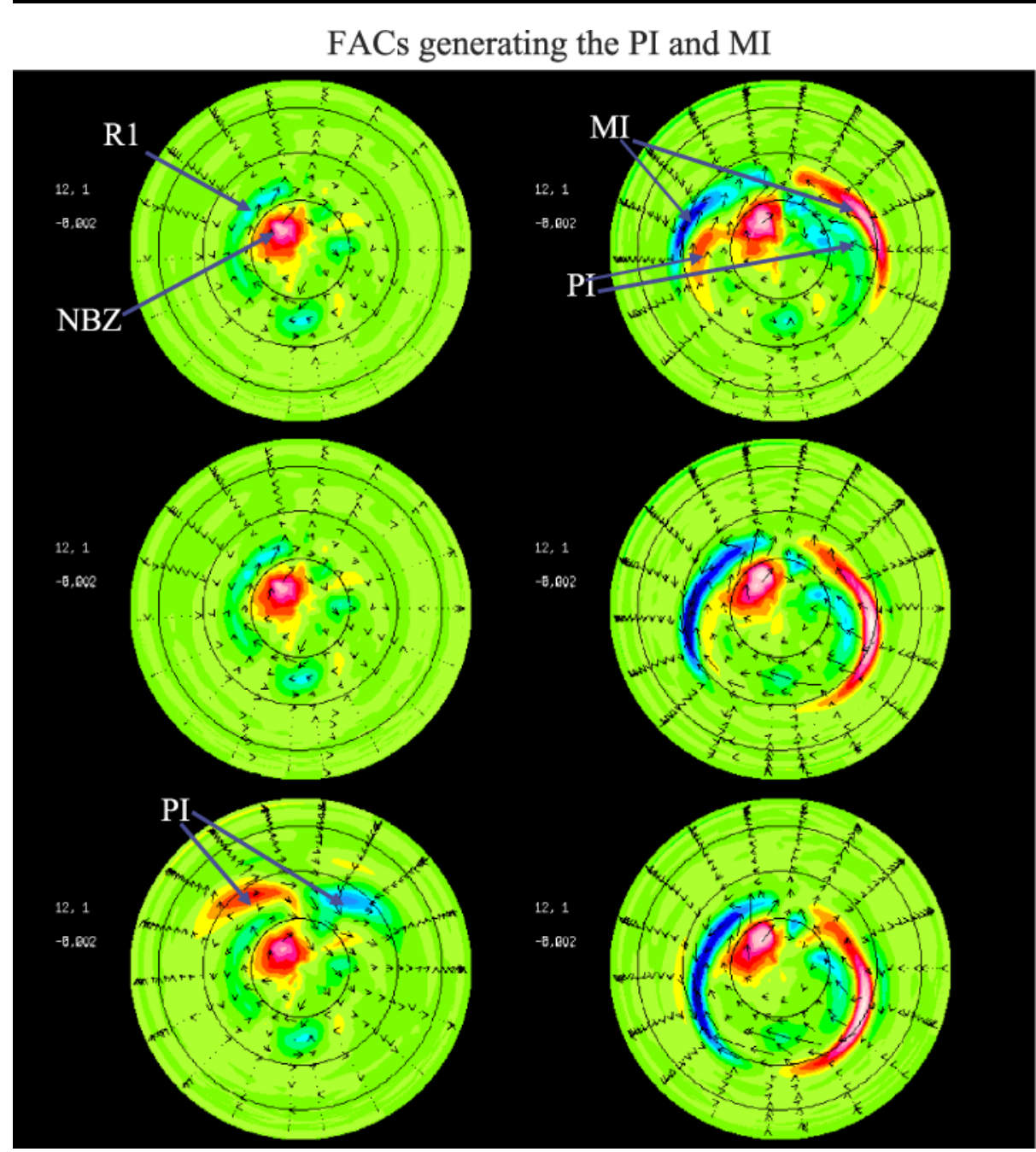

Fig. 19 FAC distribution in the ionosphere during the SC. Yellow, red, and white show downward FAC in an increasing order, and dark green, blue, and black shows upward current in an increasing order. These color codes are same to Fig. 11. Time intervals between the panels are 2 minutes. The time sequence is from top to bottom and left to right. Three circles illustrate latitude lines at $60^{\circ}, 70^{\circ}$, and $80^{\circ}$ north. The initial condition for the first panel is that shown in Figs. 15, 16, and 17. R1 and NBZ show region-1 and NBZ FACs. The PI starts at the third panel

motion and clockwise (counterclockwise) Hall current. Then as a next step, the process to generate these FACs must be clarified inside the framework shown in Fig. 10. That is equal to understanding the SC as a transient convection process in the M-I compound system.

Recent MHD simulations (Slinker et al. 1999; Keller et al. 2002; Fujita et al. 2003a, 2003b) have succeeded to numerically reproduce the SC. Many new findings are made for the nature of SC from the analyses of these numerical results. Figure 19 shows an MHD simulation result for the time sequence of FAC distribution in the ionosphere after the arrival of pressure increase. The initial condition for this MHD simulation is that shown in Figs. 15, 
16, and 17. In Fig. 19 that shows the development of FAC on the ionosphere during the SC, the time sequence is from top to bottom, and left to right. Time interval between the panels is $2 \mathrm{~min}$. In the initial state illustrated in the first panel, region-1 and NBZ FACs can be observed in the polar region. The NBZ FAC is downward on the duskside and upward on the dawnside. The duskside NBZ current is stronger than the dawnside NBZ current due to the negative IMF $B_{y}$. At this time, a weak region-1 FAC pair having an opposite polarity to NBZ FAC surrounds the low-latitude side of NBZ FAC.

Soon after the arrival of increased pressure, a pair of FAC appears in the third panel in the dayside region. At this stage, new FACs are downward in the post-noon sector and upward in the pre-noon sector. This FAC pair that generates the PI first appears in the low-latitude region, then gradually progresses to the high latitudes and finally disappears at the 6th panel. According to the shift from the PI phase to the MI phase, another FAC pair having a reverse polarity appears following the migration of FAC generating the PI. This MI FAC pair is first visible in the third panel as a weaker current pair on the low latitude side of PI current pair. According to the progress of time, the MI FACs grow to a remarkable strength, then shift their positions to those of region-1 FAC, and finally mix with the region-1 FAC pair that is downward in the dawnside and upward in the duskside. Then FACs generating the MI phase gradually become weak staying at this same position. This two step response is commonly seen in every MHD models (Slinker et al. 1999; Keller et al. 2002; Fujita et al. 2003a, 2003b).

Figure 20 exhibits the magnetospheric configuration at the PI stage showing the pressure distribution, current strength, magnetic field lines, and current lines in the dayside. In this figure, color shading and contour lines show distributions of pressure and current strength in the noon-midnight meridian and equatorial planes. In the top panel, magnetic field lines extending from the center of downward PRI current are drawn by red lines. In the second panel, current lines connected to the center of downward PRI current are shown by red lines. It is apparent in the top panel of Fig. 20 that the magnetic field line at the PRI current center is not connected to the magnetopause. This magnetic field line is closed deep inside the dayside magnetosphere. As a natural consequence, the FAC flowing on this magnetic filed line is not directly connected to the magnetopause current, but is connected to the magnetopause current via a radial current in the outer magnetosphere. This current configuration is a key structure to understand the PRI.

At the PI stage, a compressional motion is propagating within the magnetosphere following the contact of pressure impulse. Radial current is a polarization current that is formed at the propagation front of compressional wave. The current loop shown in Fig. 20 is composed of three parts, the increased Chapman-Ferraro current on the magnetopause, polarization current at the propagation front, and the FAC (Fujita et al. 2003a). The polarization current and the FAC are connected through the mode conversion from isotropic compessional mode to the transverse Alfven mode caused by non-uniformity of the medium (Tamao 1965). From these structures, the middle panel of Fig. 20 gives the answer for the PI mechanism by clarifying how a current loop is formed from the increased Chapman-Ferraro current, polarization current, and the FAC.

The third panel of Fig. 20 shows the distribution of $\mathbf{J} \cdot \mathbf{E}$ along the current loop. It can be seen from Fig. 20 that the increased Chapman-Ferraro current acts as the dynamo on which the work is done by the solar wind dynamic pressure $(\mathbf{J} \cdot \mathbf{E}<0)$. Since plasma is pushed at the front of propagating compressional wave, polarization current acts as the motor $(\mathbf{J} \cdot \mathbf{E}>0)$. These mechanical structures seen from Fig. 20 reveal the nature of PRI from the view point as shown in Fig. 10. In the PI stage, however, the magnetosphere and the 
Fig. 20 Magnetic field line (red lines in the top panel labeled by $B$ ) and current line (red lines in the middle panel labeled by $J$ and multi-colored lines in the bottom panel) traced up from the center of FAC generating the PRI. Color codes and contours show pressure and current magnitude in the noon-midnight meridian and equatorial planes. In the bottom panel $\mathbf{J} \cdot \mathbf{E}$ value is mapped on the current lines by color codes. FAC, CFC and Pol. $\mathrm{C}$ show field-aligned current, Chapman-Ferraro current and polarization current

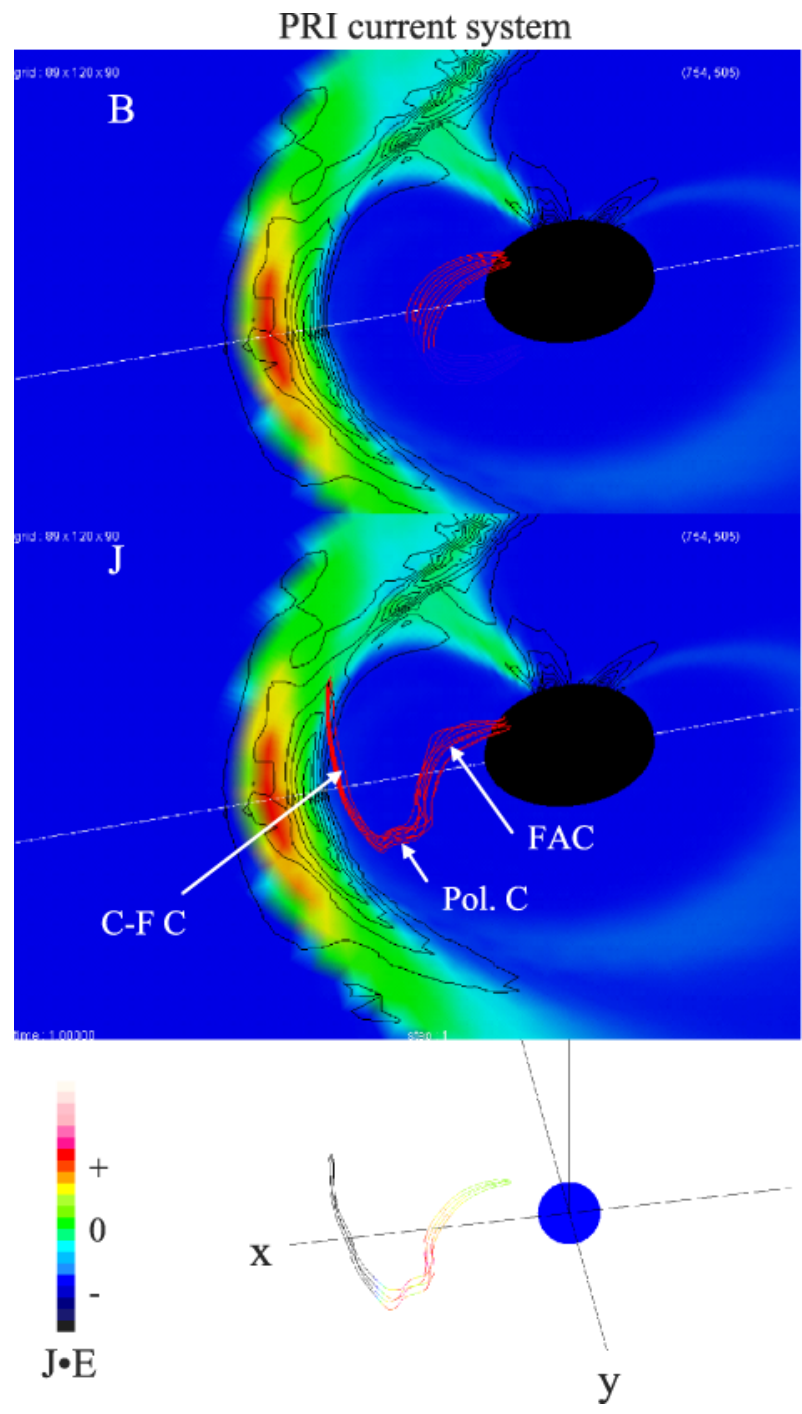

ionosphere do not fully establish the two-way interaction process. That is because the time scale of PRI is shorter than the time scale to establish the feed back loop in Fig. 10.

Figure 21 illustrates MI transient convection. The panel on the lower-right part shows the time sequence of ionospheric convection after the arrival of pressure impulse. The second panel still shows the PRI convection that is anticlockwise at the post-noon region and clockwise in the pre-noon region. After the second panel, the PI convection disappears and only the MI convection appears in the ionosphere. The MI transient convection has the same convection pattern as the reconnection-driven two-cell convection under the southward IMF condition. The MI convection reaches its peak at the third panel, and then gradually becomes slow in the following panels. The upper-left and central panels in Fig. 21 show the ionospheric convection and magnetic field structure at the timing between third and 4th panels in the lower-right part. 


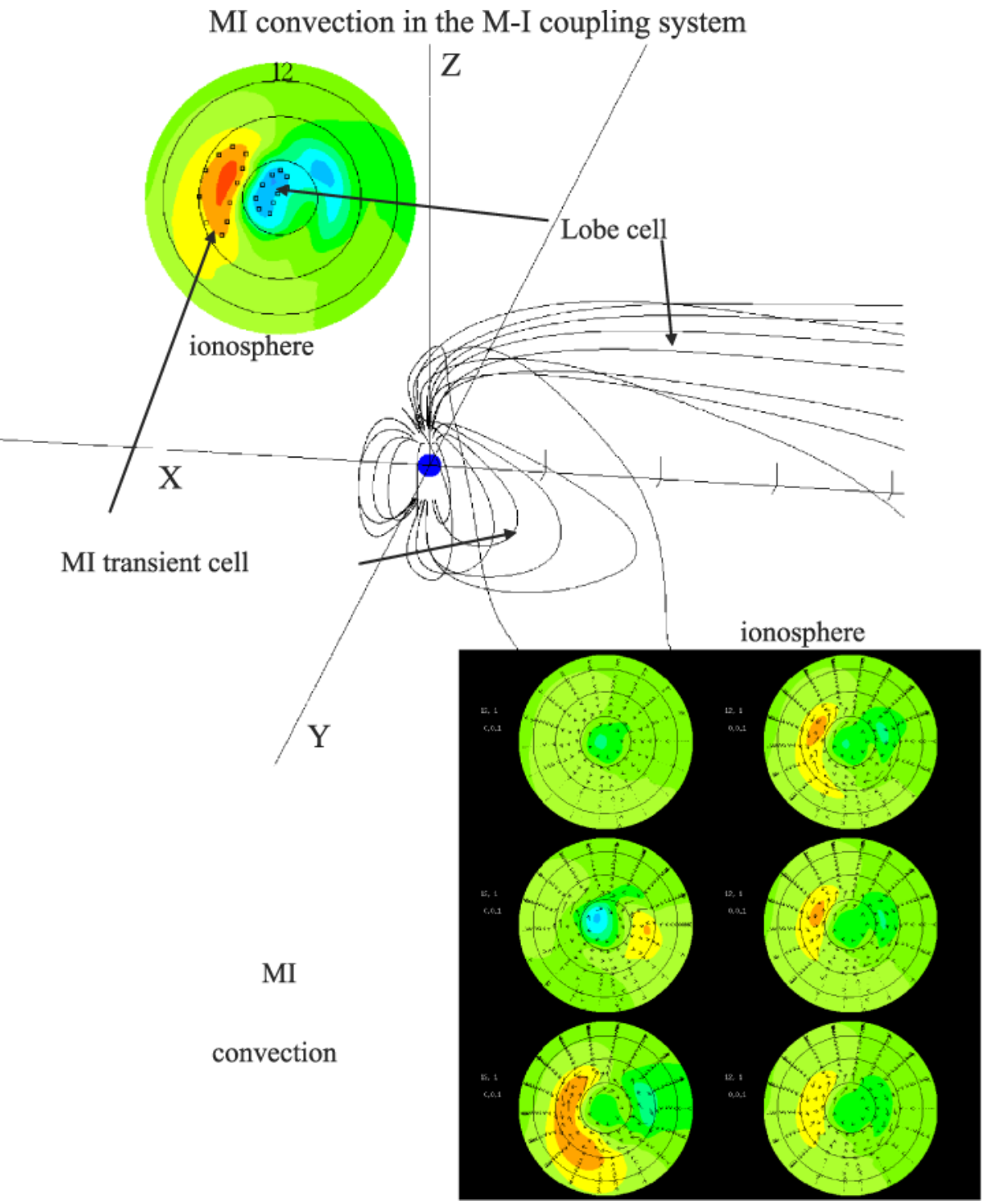

Fig. 21 Magnetic filed lines during the MI convection. Upper and lower insets show the ionospheric convection (potential) during the SC. In the upper inset, footpoints of magnetic field lines drawn in the central drawing are marked by dots. Two groups in these dots show magnetic field lines constructing the lobe cell and MI cell, respectively. The time sequence in the lower inset is from top to bottom and left to right. The timing of central drawing corresponds to the 4th panel in the lower inset when the MI convection starts to decrease

The small circles in the ionospheric convection represent the footpoints of the magnetic field lines drawn in the central panel. Small circles in the ionospheric convection are organized into two groups, one group into the high-latitude round cell and the other group into the low-latitude dusk cell. Among them, the high-latitude cell is the footpoint of lobe cell that is normally formed under the northward IMF condition (Sect. 5). Magnetic field 
lines in this cell include only open field lines. In contrast, the ionospheric convection cells at low latitudes in the dusk sector accompany the region-1 FAC and include only closed field lines. This characteristic convection during the MI phase clearly differs from mergingcell convections induced by reconnection. This convection at the MI phase constructed only with closed field lines should be called the MI transient cell. At the end of PI, the distributions of pressure and magnetic field do not match with the compressed magnetosphere, and are in a state of pressure imbalance. The MI transient convection is generated to resolve this pressure imbalance. Using this over-compression state as energy source, MI transient convection proceeds to transfer the magnetosphere into a new equilibrium state, along the framework shown in Fig. 10. The generation mechanism of FAC associated with the MI transient convection is same to that of the region-1 FAC (Fujita et al. 2003b).

The disturbance in the magnetosphere is quite severe at the initial PI stage when energy is directly supplied inside the magnetosphere through compression. Solar wind force is directly exerted inside the magnetosphere through the compressional wave. During the MI stage when this energy is consumed, the magnetospheric perturbations are rather mild. Nevertheless in the ionosphere, the PI produces only small variations, while the MI is observed as a large disturbance. This is a direct consequence of the fundamental dynamics of M-I system shown in Fig. 10. Since the PI is a compressional motion, it is basically invisible in the ionosphere (Kiverson and Southwood 1991). Due to the non-uniformity of the medium, there generates only a small disturbance as PI through the mode conversion in the magnetosphere. In contrast, the MI is a shear motion coupled between the magnetosphere and ionosphere, and can therefore be observed as a large disturbance even in the ionosphere. Thus, the difference between the PI and MI gives an instructive material to understand the basic characteristics of the M-I compound system.

\subsection{Reconstruction of Convection for Different IMF $B_{y}$ and the Theta Aurora}

During magnetically quiet intervals under northward IMF conditions, polar cap (sunaligned) arcs appear in the high-latitude ionosphere. Occasionally, polar cap arcs extend across whole of the polar cap as large-scale sun-aligned arcs. The auroral pattern made up of these transpolar arcs (TPA) and the oval has been called the theta aurora. Since the first observation of theta aurora by DE-1 spacecraft (Frank et al. 1982), this phenomenon has been of great interest for many researchers.

In general, the TPA is predominantly a northward IMF phenomenon associated with large positive IMF $B_{z}$, and greater IMF magnitude than normal (Cumnock et al. 1997; Chang et al. 1998). The precipitating electrons for the TPA are less energetic than those for the nightside auroral arcs, and called as the polar shower (Winningham and Heikkila 1974). While the polar shower is observed more frequently regardless of the TPA, the polar shower in the TPA accompanies ion precipitation. The ion precipitation and spectral characteristics of plasma associated with the TPA suggest that the TPA is on the closed field lines connected to the boundary plasma sheet (Frank et al. 1982; Obara et al. 1993).

For positive (negative) IMF $B_{y}$, the TPA moves slowly duskward (dawnward) in the northern hemisphere. That tendency shows that the TPA is a dynamically-developing structure rather than a static structure in the polar cap. The initiation process of the TPA indicates the tendency more clearly. The sign change of $B_{y}$ from negative (positive) to positive (negative) causes the initiation of TPA by splitting the auroral form from the dawnside (duskside) of the oval in the northern hemisphere (Cumnock et al. 1997, 2002). Typically, this splitting starts about 30 min after a sudden change of IMF $B_{y}$. Then, the TPA is moving toward the duskside (dawnside) when $B_{y}$ stays positive (negative) after the sign change. In the southern 
hemisphere, the location and motion of TPA is on opposite side and in opposite direction. Often, the TPA fades away after having crossed the noon-midnight line (Cumnock et al. 2002).

Associated with the IMF change during the TPA event, ionospheric convection also shows dynamical reconfiguration. For $B_{y}$ negative (positive) condition, the counterclockwise (clockwise) round cell occupies the center of the polar cap, accompanied by the smaller crescent cell on the evening (morning) low-latitude side (see Sect. 5.2). After the sign change of $B_{y}$ from negative (positive) to positive (negative), therefore, the cell structure must reconfigure so as to reverse the rotation direction of the round cell and to shift the position of crescent cell from evening (morning) to morning (evening). This reconfiguration process was related to the formation of the TPA by Cumnock et al. (1997). They associated the position of TPA with the convection reversal in the newly developed round cell. The TPA is also associated with paired upward and downward field-aligned currents (Bonnell et al. 1999). Since the convection potential is determined through the ionospheric closure process of the FAC, the convection cell must be configured having closed relationships with the FAC and stress imposed on the ionosphere (see Sect. 4). However, meanings of paired FACs are not sufficiently explained in the models so far presented.

There is not much information on what occurs in the magnetosphere during the TPA events. A leading question of the TPA phenomenon has been what structure in the plasma sheet corresponds to the TPA in the underlying ionosphere. Many models have been proposed to answer this question. A bifurcated plasma sheet model is proposed from the observation that particles from the plasma sheet boundary layer in the TPA are associated in the surrounding regions with particles normally observed in the polar cap. Kan and Burke (1985) proposed that under the due northward IMF condition the TPA evolves from midnight to noon on sunward-convecting closed-field-line region that is bifurcated from the plasma sheet and mapped down to the central polar cap. This model proposed by Kan and Burke (1985) is, therefore, a steady state model which assumes a steady reverse-cell convection in the central polar cap (see Sect. 5.2). For the due northward IMF condition, many theoretical models (Troshichev 1990) as well as recent magnetohydrodynamic (MHD) modeling results (Gombosi et al. 2000) show that the M-I convection consists of only closed field lines confined to the short tail. Since the polar-cap convection cell that includes only closed field lines coexists with a thick plasma sheet, these configurations seem not to support the theta aurora configuration suggested by Kan and Burke (1985).

In many cases, the $B_{y}$ dominant condition is observed associated with the TPA. From this fact, changes in IMF $B_{\mathrm{z}}$ or $B_{y}$ that require reconfiguration of the dayside merging are considered as a cause of the TPA. According to the antiparallel merging structure (Crooker 1979), the dayside merging points change their position depending on the IMF orientation (see Sect. 5). The merging location moves closer to the equator associated with $B_{z}$ negative excursion (Newell and Meng 1995), or jumps to other side of magnetic noon associated with a $B_{y}$ sign change (Chang et al. 1998). Associated with the relocation of the merging point to a new position, a new open-field-line bundle begins to form, repelling a preexisting openfield-line bundle. If the merging-line jump on the dayside magnetopause is so significant that the newly opened region is detached from the previous open region, the new open-field-line region must spread pushing away the preexisting closed-field-line region in the ionosphere. At this stage, a closed region remains between new and old open-field-line regions. As the new open flux continuously fills the polar cap, a strip of closed-field-lines shifts further poleward to generate the TPA (Chang et al. 1998). Thus, the TPA can be formed along the closed field line region that separates old and new open sections. In this model, a contracted polar cap is more suitable before the theta aurora initiation, because the newly opened region 
must be well separated from the previously open region. Chang et al. (1998) pointed out another condition for theta aurora initiation that $\left|B_{y}\right|>\left|B_{z}\right|$ must continue for $\sim 10 \mathrm{~min}$ after the IMF change.

As shown in Sect. 5, a nonzero IMF $B_{y}$ causes a twist of the tail plasma sheet. Seen from the tail side, twist is clockwise for $B_{y}$ positive and counterclockwise for $B_{y}$ negative. Kullen (2000) took notice of this point and proposed a TPA generation mechanism that considers the topological development of the magnetosphere as the main cause to generate the TPA. Kullen (2000) assumed a sign change of IMF $B_{y}$ that must cause the rotation of magnetotail to twist the whole tail in the opposite direction. In the midst of rotation, magnetotail is expected to configure such that the near earth tail and the far tail are twisted in opposite directions. Under such assumption, Kullen (2000) showed from a modified Tsyganenko model that mapping from the tail current sheet to the ionosphere shows a separate region of closed field lines that extend to the polar cap.

Slinker et al. (2001) first applies the global MHD simulation to the formation of theta aurora and showed that the TPA is formed below closed field lines that break off from the main oval and convect across the polar cap. Their simulation seems to support the results of Chang et al. (1998). Also their results confirmed the previous expectation that closed field lines on the TPA are connected in the magnetosphere to the closed field finger that separates two open lobes. Figure 22 shows a time sequence of the pressure distribution in the polar ionosphere in a numerically reproduced theta aurora event. This simulation result is obtained by changing IMF $B_{y}$ at $t=0$ min from minus (dawnward) to plus (duskward) under the northward IMF condition with $|B|=15 \mathrm{nT}$. The time sequence is from left to right and top to bottom. The first panel exhibits the pressure distribution in the initial state for IMF $B_{y}$ negative. In this panel, the high pressure area can be looked upon as the projection of return flow region in convection threaded by closed field lines. The second, third, and 4th panels illustrate pressure distributions at $t=9 \mathrm{~min}, t=15 \mathrm{~min}$, and $t=24 \mathrm{~min}$, respectively. In the third panel, a small low-pressure region appears in the inflated dawnside high-pressure region. As time goes on, this low-pressure region becomes more dominant and a high pressure region detaches from dawnside oval. A theta aurora has fully formed in the 4th panel. At this time, however, the TPA seems to be separated into two parts, namely a nightside extending part and a dayside extending part. Movement of transpolar arc is from dawn to dusk. This sequence of TPA formation in the MHD simulation reproduces the observed features shown by Cumnock et al. (1997) quite well.

A key point to get the result shown in Fig. 22 is how to determine the inner boundary condition in MHD calculations (see Sect. 2.3). In the MHD simulation, the inner boundary condition for pressure is determined so as to project down pressure values along field lines from the magnetosphere to the ionosphere for downward (precipitating) flow region, and to give a low pressure value for upward (polar wind) flow region. This method to determine the boundary condition acts on the principle of characteristic lines for hyperbolic equations. According to the flow direction on the inner boundary, number of fixed variables must be changed so as to match with the number of characteristic lines directed toward the calculation domain. Thus, high-pressure regions in Fig. 22 are formed as the projection of high-pressure region in the magnetosphere.

Figure 23 shows plasma sheet signatures corresponding to Fig. 22. This figure shows mapping plots of temperature in the tail cross section at $x=-15$ Re seen from downtail. In the first panel of Fig. 23, two kinds of high-temperature area are seen in the center of the magnetosphere and on the magnetopause, corresponding to the plasma sheet and the sash structure (White et al. 1998), respectively. White et al. (1998) combined high-temperature 


\section{Formation of theta aurora}

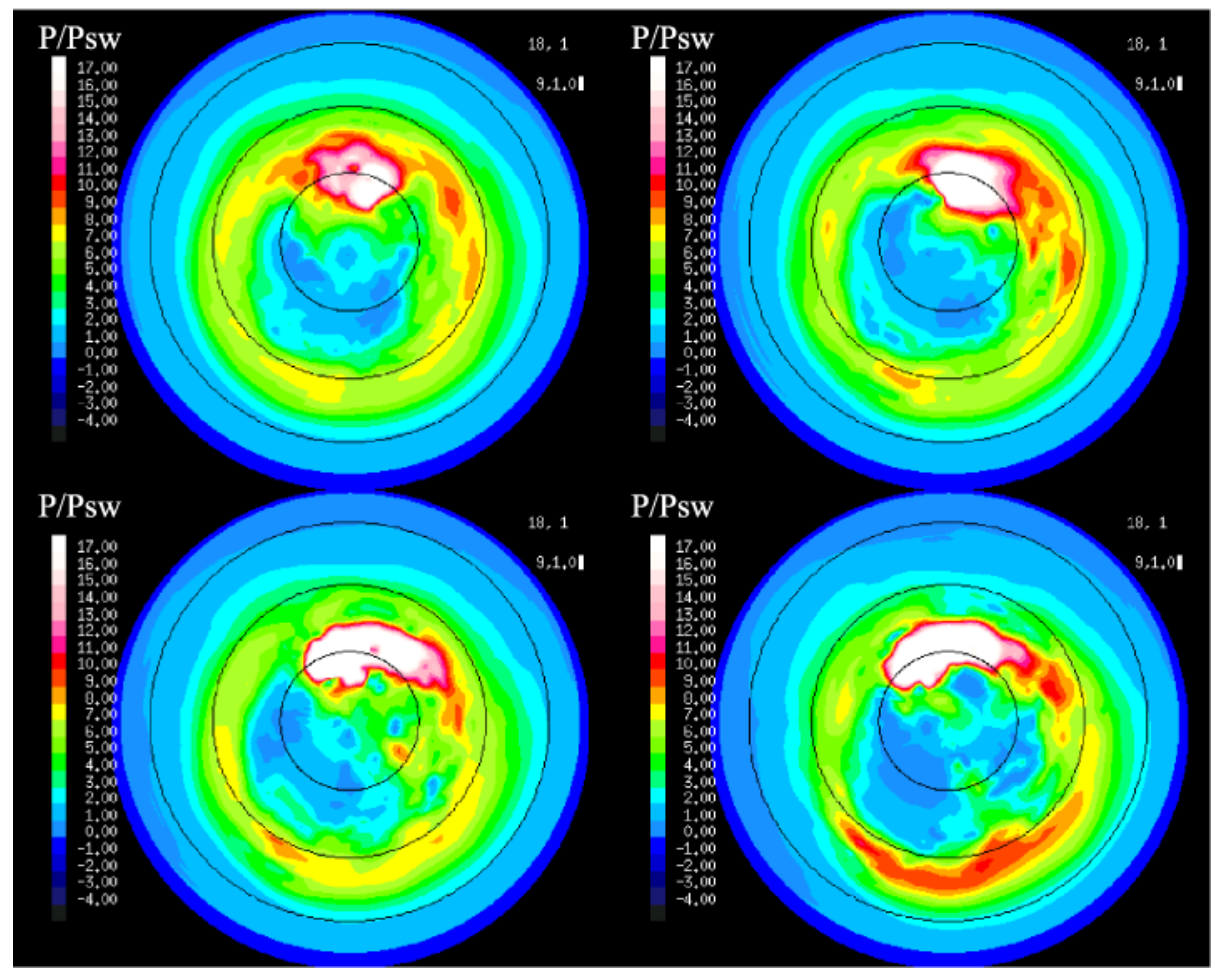

Fig. 22 A time sequence of four pressure distributions in the polar ionosphere at $t=0,9,15$, and 24 min. Pressure values are normalized by the solar wind pressure $\left(P_{\mathrm{sw}}=47 \mathrm{pPa}\right)$. Noon is to the top, and three circles show northern latitudes at $60^{\circ}, 70^{\circ}$, and $80^{\circ}$. Time goes on from left to right and top to bottom. Detachment of high-pressure region from the dawnside oval starts at the third panel. Drifting toward dusk, detached regions grow into the TPA

plasma sheet with high-temperature areas in the sash to call the whole structure the crosstail S. High temperature in the sash indicates the reconnection process occurring on the magnetopause (Tanaka 1999) while high temperature in the plasma sheet indicates the formation of closed field lines. In the initial state shown in the first panel, counterclockwise skew of plasma sheet is seen as an effect of negative IMF $B_{y}$ (Crooker et al. 1998; Nishida and Ogino 1998; Tanaka 1999). After the change of IMF direction, the first signature of the positive IMF $B_{y}$ effect is observed as a thickening of the plasma sheet (second and third panels). This thickening suggests an accumulation of closed magnetic field in the plasma sheet. After the sign change of IMF $B_{y}$, old lobe fields connected dawnward or $B_{y}$ negative IMF must be replaced by new lobe fields connected duskward or $B_{y}$ positive IMF. However, this replacement cannot be achieved instantaneously but it must proceed gradually from the outer part of the lobe to the core part near the plasma sheet (Tanaka 1999, 2000b). At the early stage soon after the change of IMF $B_{y}$, therefore, the lobe regions are still occupied by old open field lines connected to the old IMF fields. They continue to generate closed field lines even during $t>0$ through the tail reconnection process, to supply closed field lines to the plasma sheet. 


\section{Plasma sheet in the yz plane}

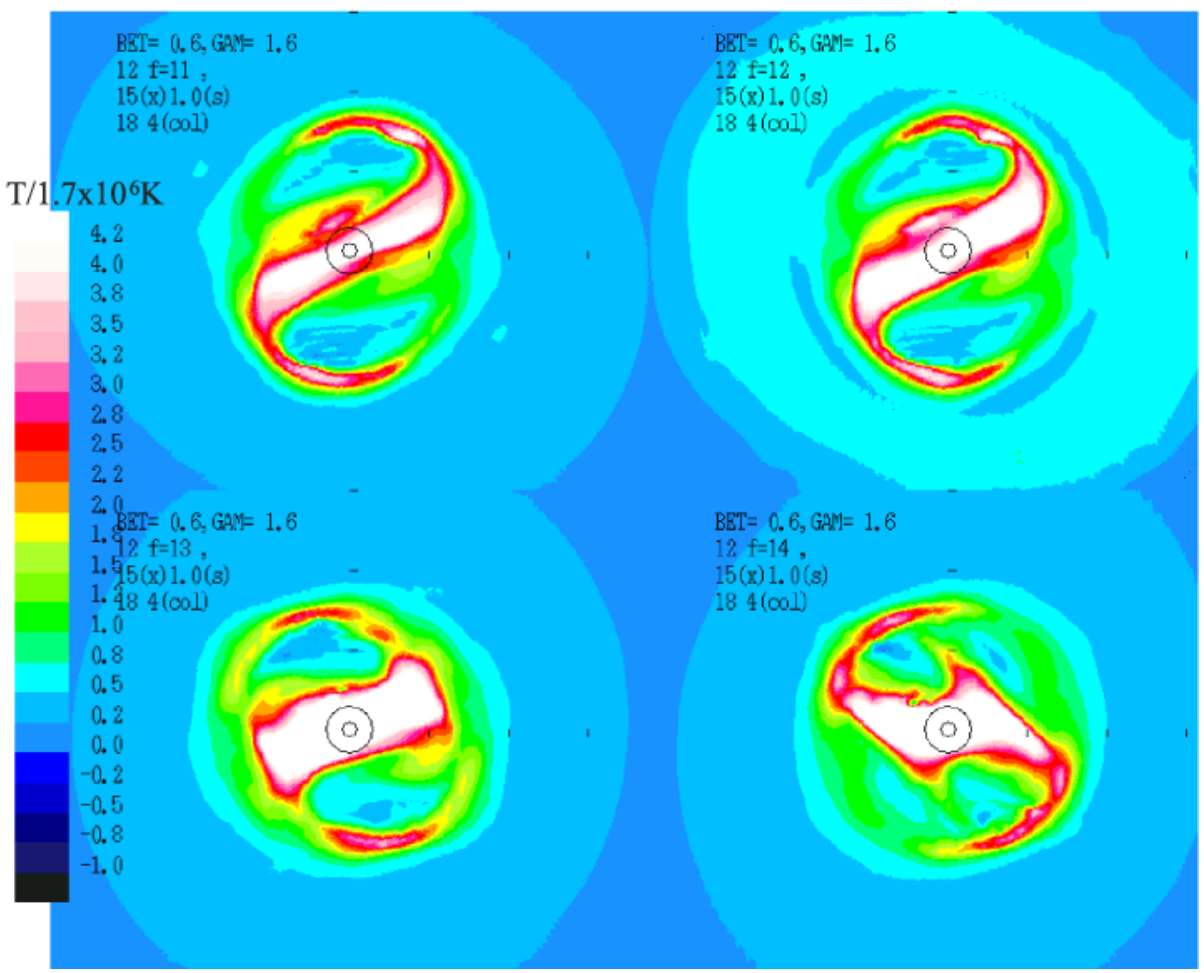

Fig. 23 Mapping plots of temperature in the tail cross section at $x=-15 \mathrm{Re}$ seen from downtail. Temperature values are normalized by the value of $1.7 \times 10^{6} \mathrm{~K}$. Time sequence is from left to right and top to bottom. Two circles show the sizes of the earth and inner boundary at $r=3 \mathrm{Re}$. Plasma sheet signatures leading to the formation of TPA are observable in this plate

In the third panel, the lobes at $x=-15 \mathrm{Re}$ are still occupied by open field lines connected to the old IMF ( $B_{y}$ negative or dawnward) although its shape is modified and neighboring plasma sheet is thickened to a large extent. After reaching a peak in thickening in the third panel, the plasma sheet exhibits a kink structure in the 4th panel. Corresponding to the kink structure, new lobe appears separated from old lobe on the dawnside (duskside) in the northern (southern) hemisphere. As will be seen from Fig. 24, this new lobe consists of open field line connected to the new (duskward or $B_{y}$ positive) IMF. Between old and new lobes seen in the 4th panel, high-temperature regions bifurcating from the kink structures extend as far as the magnetopause. At the same time, the cross-tail S reverses its polarity. It is apparent from this panel that the twist of plasma sheet to the opposite direction does not proceed from near earth tail to distant tail as expected by Kullen (2000) but proceeds from outer surface of the tail to core part of the tail. As a result, the cross-tail $\mathrm{Z}$ is formed in the plasma sheet. It is quite natural to consider that the splitting oval seen on the dawnside in Fig. 22 is connected to the new lobe, and the TPA is connected to the bifurcated plasma sheet extending from the kink point.

Figure 24 shows the global configuration of magnetic field lines convecting in the magnetosphere during the formation of TPA (at $t=24 \mathrm{~min}$ ). This figure gives a view from 


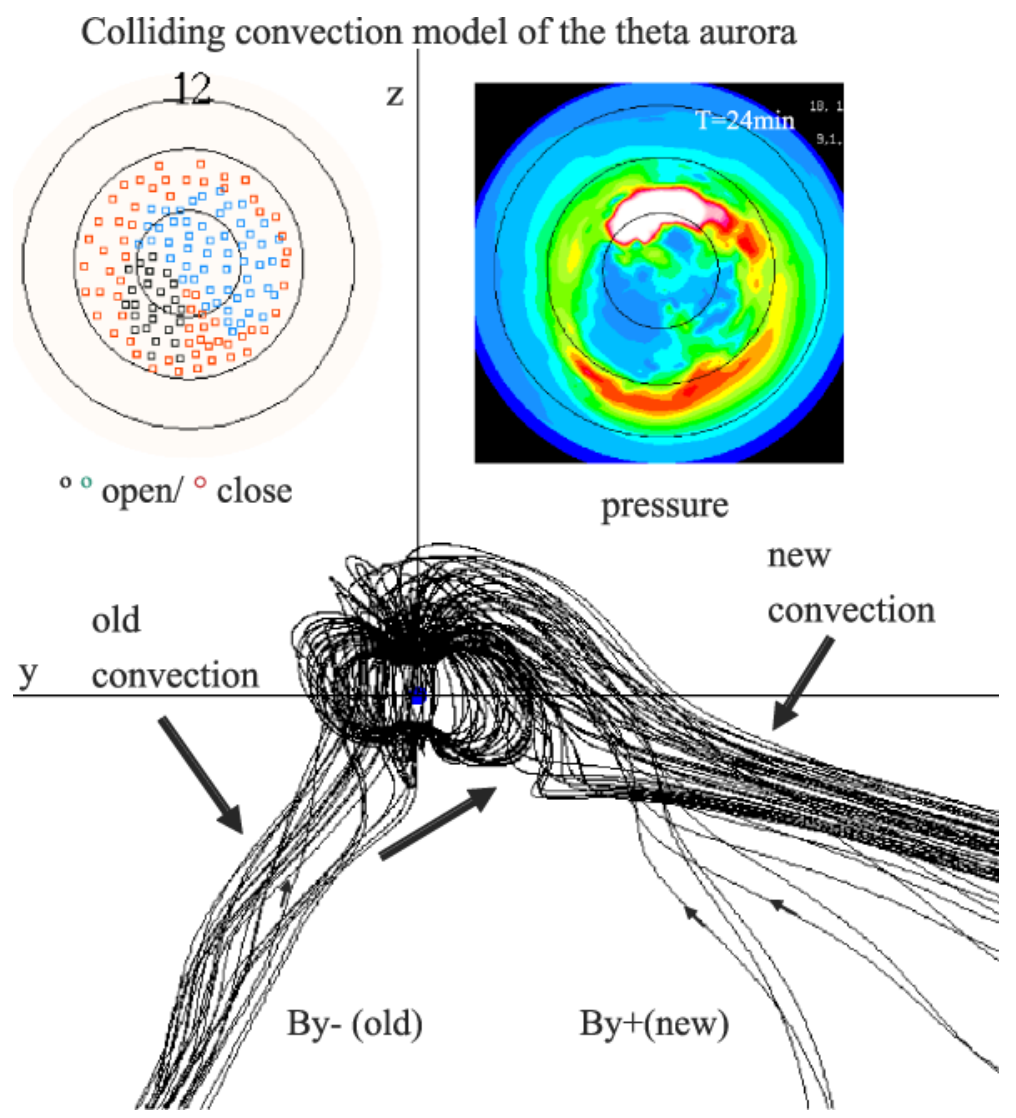

Fig. 24 Field lines traced up from the polar ionosphere under a development of the TPA $(t=24 \mathrm{~min})$. The view is from the downtail. A blue sphere shows the size of the earth. Two upper insets show footpoints of field lines (left) and the reproduction of 4th panel in Fig. 22 (right). Footpoints are shown by red, black and blue points for closed, old open and new open field lines. Arrows schematically show the convection

downtail of field lines traced up from the polar ionosphere under the full development stage of the TPA. Two upper insets in Fig. 24 show footpoints of field lines (left) and the reproduction of 4th panel in Fig. 22 (right). In the left inset, red, black, and blue points show footpoints of closed field lines, open field lines connected to the negative (old) IMF $B_{y}$, and open field lines connected to the positive (new) IMF $B_{y}$, respectively. While the region between the TPA and the duskside auroral oval includes open field lines connected to the old (dawnward or $B_{y}$ negative) IMF, the region between the TPA and the dawnside auroral oval includes open field lines connected to the new (duskward or $B_{y}$ positive) IMF. Between these two different kinds of open field lines, closed field lines are emerging in Fig. 24 extending deep into the tail. On the polar ionosphere shown in the upper left inset, these distributions of closed field lines are well reflected in the distribution of red spots. While red spots are primarily distributing in low latitudes so as to surround the black and blue spots in high latitudes, a part of red spots is forming a bulge that extends from the nightside oval to the polar cap. The result shown in Fig. 24 is same as the previous report by Slinker et al. (2001). Closed field lines traced up from this bulge extend as far as $-40 \mathrm{Re}$ in the tail region. 


\section{Closed field lines above the TPA}

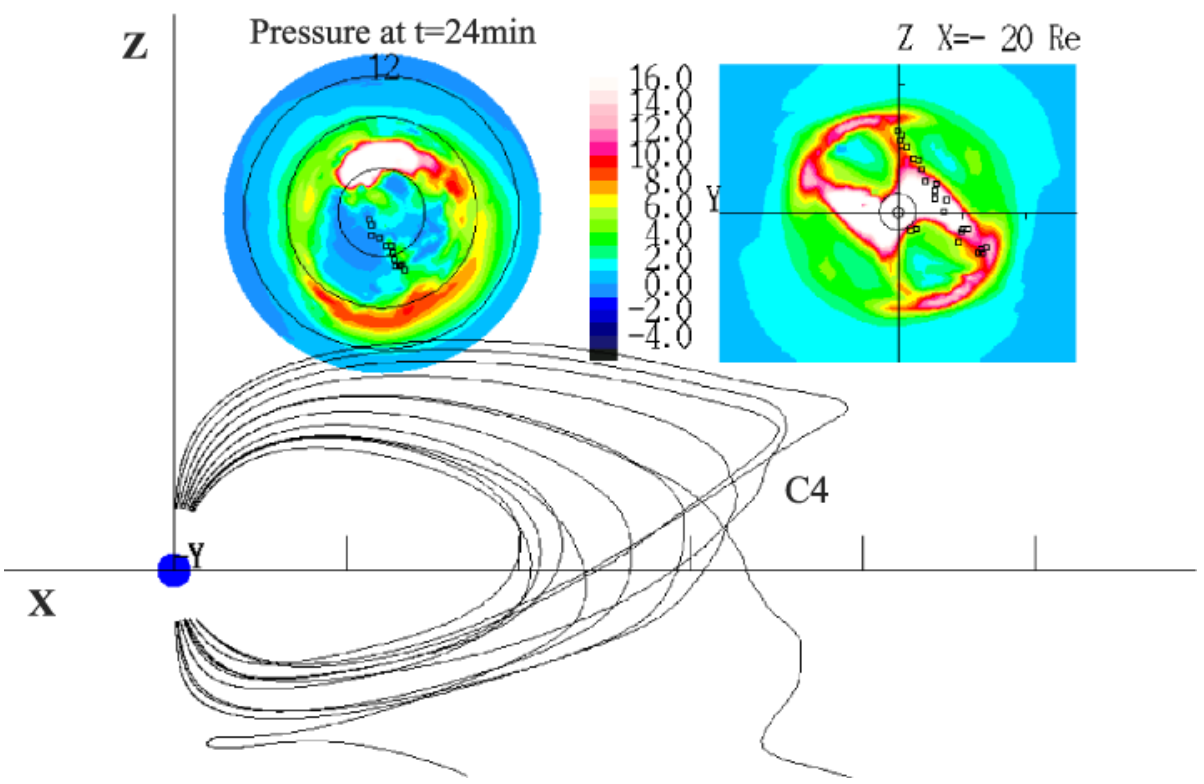

Fig. 25 Closed field lines traced up from the TPA viewed from the duskside. Two upper insets show by dots footpoints of field lines on the northern polar ionosphere (left) and cross points of closed magnetic field on the $y z$-plane at $x=-20 \mathrm{Re}$ (right). The color codes in the upper left and right insets show pressure (same to the last panel of Fig. 22) and temperature (same to the last panel of Fig. 23), respectively

Comparing with the upper right inset, the TPA is seen at the same position of this closed field line bulge.

Figure 25 shows the structure of closed field lines above the TPA viewed from the duskside. In the upper left inset, footpoints of closed field lines drawn in the main part of Fig. 25 are shown by dots on color codes showing the pressure, and in the upper right inset, cross points of close field lines with $y z$ plane at $x=-20$ Re are shown by dots on color codes drawing the temperature. In Fig. 25, the further the field line is extending deep into the tail, the more recently it was reconnected in the plasma sheet. In the inclined plasma sheet tail, tail reconnection occurs not in the central part $(y=0)$ of the plasma sheet but in the both flanks of the plasma sheet (Tanaka 1999). The feature seen in Fig. 25 matches this result quite well. It is also apparent from Fig. 25 that the recently closed field lines occupy the sunward part of TPA. We get here the most important result that the TPA formation is not an extension from the oval but it is an accumulation to the oval. In the upper right inset, closed field lines trapped inside the plasma sheet are pressed by the appearance of new lobe.

\subsection{Colliding Convection Model of the Theta Aurora}

In the TPA model presented in the previous subsection, the closed field lines that develop in association with the TPA are generated in the plasma sheet. The merging cell is primarily responsible for the generation of these closed field lines in the plasma sheet. For negative IMF $B_{y}$, convection on the northern round cell is counterclockwise (Fig. 16). In the northern round cell, open field lines are transported tailward along the convection path on the dusk 
side of round cell (solid curves in Fig. 16, $1 \rightarrow 2 \rightarrow 3 \rightarrow 4$ ). After the nightside reconnection, resulting closed field lines return toward the dayside through the dawnside flank of the magnetosphere (dotted curves in Fig. 16, $4 \rightarrow 1$ ). The southern counterparts of these closed field lines are on the exchange cell in the evening and on the crescent cell in the morning (mirror image of Fig. 17). In the southern crescent cell that is situated on the morning side under negative IMF $B_{y}$ conditions, open field lines after the second reconnection (see Fig. 18) convect tailward along the convection path on the high-latitude side of crescent cell (solid curve). After tail reconnection, closed field lines connected to the southern crescent cell are transported toward the dayside along the dawn flank. The northern counterparts of these closed field lines are on the round cell (dotted curve in Fig. 16). However, this closed field line region cannot return to the dayside readily. The southern footpoint must change from the crescent cell to the exchange cell (dashed curve in the mirror image of Fig. 17). These convection structures maintain their consistency in the M-I system with an inclined plasma sheet. Seen from the tailside, the twist of the plasma sheet is counterclockwise for negative IMF $B_{y}$ (first panel of 23). In these convection structures, closed field lines that develop along the TPA in the two hemispheres are those on the round cell.

Even after the sign change of IMF $B_{y}$, the old merging cell still continues to generate closed field lines that must return to dayside against the new convection pattern (dotted curve in Fig. 16). A change in the configuration of dayside reconnection, however, immediately blocks the return path by ceasing the two step reconnection. The reconnection point changes from prenoon (postnoon) to postnoon (prenoon) in the northern (southern) hemisphere. As new open field lines connected to new IMF are accumulated in the tail, a return path of closed fluxes in the old merging cell to the dayside is further blocked by the development of the new lobe structure that makes the plasma sheet twist to the opposite direction. The new northern lobe and merging cells are mirror symmetry to Figs. 15 and 16, and accumulate open fields in the northern dawn flank that is the return path of old northern round cell (Fig. 16). Thus, an accumulation of closed fluxes is enforced by the development of new lobe structures that block the return path of closed fluxes. As pointed out by Kullen (2000), the development of new lobes must result in the rotation of plasma sheet to twist it in the opposite direction. Contrary to the expectation of Kullen (2000) that the near earth tail and the distant tail are twisted in the opposite direction, the flank and core plasma sheets are twisted in the opposite direction (Fig. 23). As a result, this twist to the opposite direction (cross-tail Z) causes a kink structure between the central and side plasma sheet. This kink is the very position where closed-field-lines accumulate to and the plasma sheet bifurcates from. This kink of the plasma sheet is the blocking point of the return path in the old convection.

The theta aurora is understood as a transient response of convection to the flipping of IMF $B_{y}$. The primary controlling factor for the theta aurora generation is the topological structure of colliding convection (arrows in Fig. 24). From this example again, we can recognize the magnetospheric physics as a science of topology. The processes reproduced during the transient convection include a replacement of lobe field lines from old-IMF-originating fields to new-IMF-originating fields, a rotation of plasma sheet to opposite inclination, and a reformation of ionospheric convection cells. In the midst of these reconfigurations, a requirement that old and new convections must coexist in the $\mathrm{M}-\mathrm{I}$ system occurs to result in the formation of TPA. All these processes concern the global topological of the convection system. Thus, without considering the global topology, theta aurora mechanism cannot be interpreted successfully. Consequently, the theta aurora model shown in this section can be called "the colliding convection model". 


\section{Convection Development Leading to the Substorm}

One of the most important problems in the physics of Earth's magnetosphere concerns the cause-effect relationship which controls the substorm process. This section tries to seek the answer to this question by investigating the development of magnetospheric convection that is inevitable under the condition of southward IMF. As shown in previous sections, magnetospheric convection is the basic structure for the expression of mechanical process in the Earth's magnetosphere. It represents a process in which plasma and magnetic field in the magnetosphere and the ionosphere realize a quasi-circular motion without a considerable divergence or convergence at all altitudes, by recognizing their relative motion through the exchange of the FAC. Associated with the substorm, three phases can be identified; the growth phase, the expansion phase, and the recovery phase (Baker et al. 1996). Analyzing the development of convection, this section gives new interpretations for two key features of the substorm, namely the plasma sheet thinning during the growth phase and the dipolarization associated with the expansion phase. The most important question addressed in this section is "What is responsible for the discontinuous behavior of the M-I system that characterizes the substorm onset".

\subsection{Numerically Reproduced Substorm}

A northward-to-southward turning of the IMF enhances dayside reconnection due to its antiparallel-merging characteristic (see Sect. 5). Observations have revealed that the progress of magnetospheric convection accompanies a thinning of the plasma sheet and enhanced two-cell convection in the ionosphere accompanied by a gradual increase in current intensity. This enhanced convection represents well-known features of the growth phase (Baker et al. 1996). As explained in Sect. 6, it takes the ionosphere only approximately 5 min to turn into a two-cell convection involving the nightside, despite the prediction that at least $30 \mathrm{~min}$ is required for the enhanced magnetospheric convection to reach the nightside depending on the solar wind velocity. This is clearly the result of selective generation of shear motion by the incompressible ionosphere.

The expansion phase starts with a sudden brightening of the equatorward-most pre-onset arc, accompanied by an enhancement of electrojet activities. After the initial brightening, the region of intensified aurora moves poleward and westward (Akasofu 1964; Elphinstone et al. 1996). In the near-earth plasma sheet where the ground onset position is mapped back, the expansion onset is signified by the dipolarization of the tail field (Lopez and Lui 1990; Lui 1996). The dipolarization accompanies the plasma injection into the inner magnetosphere, the current disruption (CD), and the formation of the current wedge. When a spacecraft is located in the near-earth tail during the dipolarization, a high level of magnetic-field fluctuation is observed that is associated with the CD events (Takahashi et al. 1987; Ohtani et al. 1998). In the middle tail, fast tailward plasma flows threaded by the southward magnetic field are often observed beyond 20-25 Re (Nagai et al. 1994). These observations suggest the tail reconnection located at 20-25 Re. Flows in the near-earth region between 9 and $19 \mathrm{Re}$ are observed as a form of bursty bulk flow (BBF) indicating that the tail reconnection is patchy-bursty (Baumjohann et al. 1990b; Angelopoulos et al. 1992).

Following the expansion phase, the recovery phase appears as the decay of the current wedge, recovery of the magnetic field configuration, tailward retreat of the neutral line, plasma sheet expansions over a large range of $x$, and energetic particle flux enhancement at the geostationary orbit (Baker et al. 1996). The flow in the thickened plasma sheet is earthward (Lui et al. 1977). During many recovery phases, a double oval is formed with a 
Fig. 26 A sequence of simulated pressure $(P)$ distribution in the meridian plane of the magnetosphere. The pressure values are normalized by the solar wind pressure

$\left(P_{\mathrm{SW}}=47 \mathrm{pPa}\right)$. The size of the earth is shown by black spheres. Marks on the $-x$-axis are $10 \mathrm{Re}$ apart. Time is measured after the southward turning of the IMF. Thinning and depolarization sequence is observable in this figure

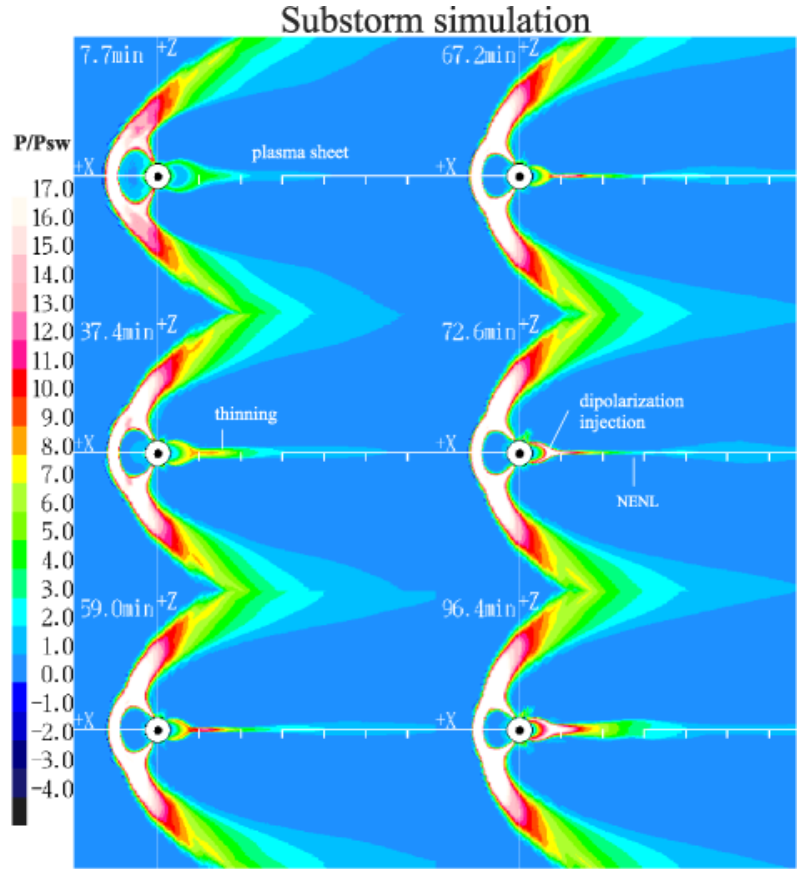

Progress in pressure distribution after

a southward turning of IMF at $0 \mathrm{~min}$

few-degrees-wide gap (Elphinstone et al. 1996). In the distant tail, retreating plasmoids are observed as bipolar $B_{z}$ structures with core $B_{y}$ field.

In recent years, substorm features are reproduced by MHD simulations to a certain extent (Tanaka 2000b). In these studies, careful considerations must be given for the dependence of simulation results on the initial condition, since the initial state for the substorm simulation must be a stationary state for quiet magnetosphere. The most general configuration that satisfies this condition may be the magnetospheric configuration shown in Sect. 5.2 (under the northward IMF condition with nonzero IMF $B_{y}$ ). Starting from the solution shown in Sect. 5.2 and changing the IMF input from northward to southward, observed sequence of substorm presented above were numerically reproduced by Tanaka (2000b). Figure 26 shows a sequence of simulated pressure distributions (normalized by the solar wind pressure $\left.P_{\mathrm{sw}}\right)$ in the meridional plane of the magnetosphere. The temporal development of the tail configuration after a southward turning of the IMF can be observed from this figure. Figure 27 shows similar results for $-V_{x}$ distributions (normalized by the solar wind sound velocity $C_{\mathrm{s}}$ ). The temporal development of the tail configuration during the substorm can be observed again from a different angle.

The first panel of Fig. 26 at $t=7.7$ min illustrates the magnetospheric configuration soon after an IMF southward turning. At this time, a thick and low-pressure plasma sheet is still observed without a noticeable effect of the southward IMF. The flow structure in the first panel of Fig. 27 indicates that the $\mathrm{x}$ line is situated beyond $x=-60 \mathrm{Re}$ shortly after the southward turning of the IMF. This structure, which is generally called the distant neutral line, is a continuation of the tail structure under the northward IMF condition shown in Sect. 5. At this time, sunward flow in the plasma sheet is still fairly slow. In the first panel of 


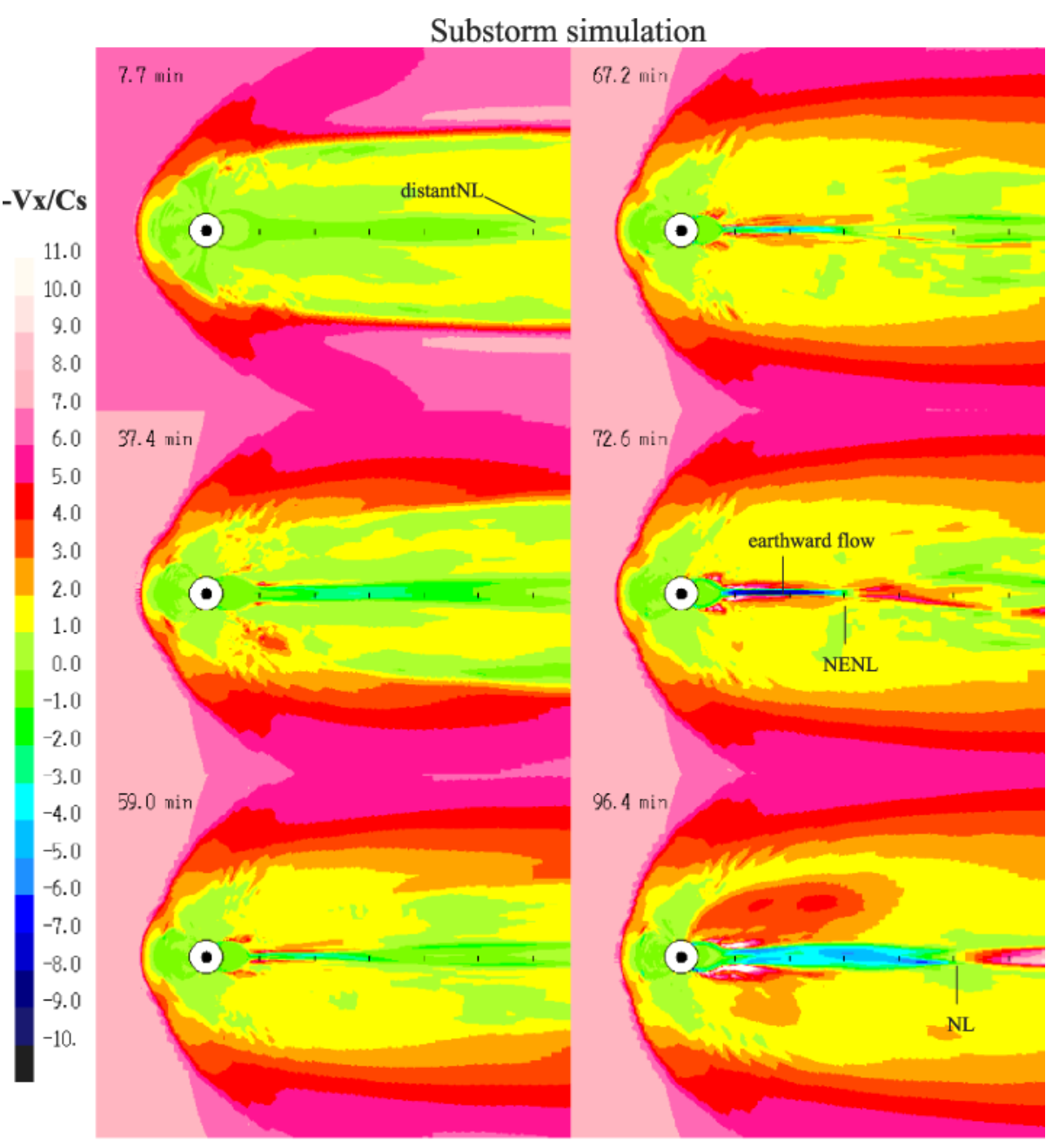

Progress in -Vx distribution after

a southward turning of IMF at $0 \mathrm{~min}$

Fig. 27 A sequence of simulated velocity $\left(-V_{x}\right)$ distribution in the meridian plane of the magnetosphere. The velocity values are normalized by the solar wind sound velocity $\left(C_{\mathrm{S}}=53 \mathrm{~km} / \mathrm{sec}\right)$. The size of the earth is shown by black spheres. Marks on the $-x$-axis are $10 \mathrm{Re}$ apart. Time is measured after the southward turning of the IMF. Neutral line position shits from distant to near earth tail

Fig. 27, fast tailward flows seen on the magnetopause beyond $x=-32$ Re are generated by the tension of disconnected field lines associated with the lobe cell circulation (see Sect. 5.2).

In Figs. 26 and 27, the thinning of the near-earth plasma sheet (growth phase) develops continuously until the $3 \mathrm{rd}$ panel at $t=59.0 \mathrm{~min}$. As thinning proceeds, earthward flow increases its speed in the near-earth tail. During this interval, the blue area in the dayside magnetosphere seen in Fig. 26 tends to shrink slightly and the flaring angle of the tail lobe tends to increase considerably, due to an erosion effect. In the growth phase (the 2 nd and 3 rd 
panels of Fig. 26), the pressure maximum in the plasma sheet exists around $x=-12$ Re. The $x$-dependence of the pressure profile in the plasma sheet inside $x=-20 \mathrm{Re}$ does not change severely between the 2 nd and 3rd panels of Fig. 26, even though the absolute value increases according to the development of thinning.

After the 3rd panels of Figs. 26 and 27, some qualitative changes occur in the tail configuration. At first, a remarkable tailward flow appears in the midtail at $t=61.8 \mathrm{~min}$. While the flow inside $x=-60 \mathrm{Re}$ is earthward throughout the growth phase as shown in Fig. 27, flow around $x=-39$ Re changes tailward at this time $(t=61.8 \mathrm{~min})$. Associated with this tailward flow, negative $B_{z}$ appears around $x=-34$ Re. About these results, details will be shown later. The next change is a start of the dipolarization. To show the thinning and dipolarization sequence, the solid curve (labeled by $\mathrm{N} \rightarrow \mathrm{S} \rightarrow \mathrm{S}$ ) in the lower panel of Fig. 28 illustrates the development of $B_{z}$ component at $x=-6.6$ Re in the midnight equatorial plane (at the midnight geosynchronous orbit). A noticeable thinning starts after about $10 \mathrm{~min}$ from a southward turning of the IMF (this timing is shown by the vertical dashed line labeled by $\mathrm{N} \rightarrow \mathrm{S}$ ). As shown in Fig. 28, the $B_{z}$ component at the midnight geosynchronous orbit decreases continuously during the growth phase. This growth-phase signature continues for about 50-minutes until a dipolarization which starts at $t=67.2 \mathrm{~min}$. After $t=67.2 \mathrm{~min}$, decreasing tendency of $B_{\mathrm{z}}$ at the midnight geosynchronous orbit changes to increasing. We define the onset by the start time of $B_{z}$ increase at the midnight geosynchronous orbit at

Fig. 28 Magnetic configuration during the growth phase (upper) and development of $B_{z}$ at the midnight geosynchronous orbit showing the thinning and depolarization process (lower). The upper inset in the upper panel shows the ionospheric convection. It is seen in this inset that the two cell convection is developed to a certain extent. The vertical dashed line labeled by $N \rightarrow S$ shows the timing of southward turning of the IMF. The vertical dashed line labeled by $S \rightarrow N$ shows the timing of northward re-turning of the IMF. The curve labeled by

$N \rightarrow S \rightarrow S$ is the result for case of no northward re-turning (case1) the curve labeled by $N \rightarrow S \rightarrow N$ is the result for the case of northward re-turning (case 2)

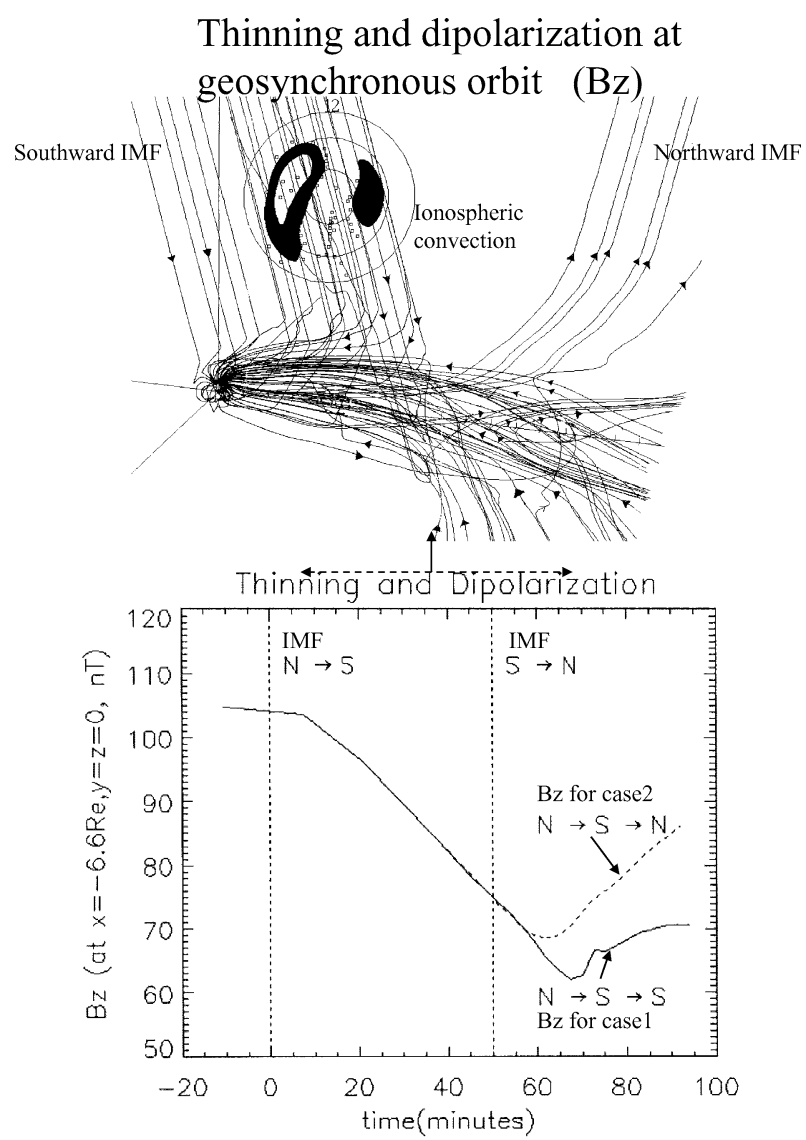


$t=67.2$ min. Under a continuously southward IMF, dipolarization tends to saturate after 10 min. In the lower panel of Fig. 28, dashed curve (labeled by $\mathrm{N} \rightarrow \mathrm{S} \rightarrow \mathrm{S}$ ) shows the case of northward returning of IMF at $t=50.0 \mathrm{~min}$ (this timing is shown by the vertical dashed line labeled by $\mathrm{S} \rightarrow \mathrm{N}$ ). In this case, the dipolarization continues without saturation. In addition, onset timing is advanced to show the substorm triggered by the northward turning of the IMF. The dipolarization is hastened 5 min compared with the steady southward IMF case. A northward re-turning of the IMF results in the deceleration of the ionospheric convection. Lyons (1995) explained the hastened onset as shown in Fig. 28 by the penetration of weakened interplanetary electric field. However, this explanation is a mixed use of EJ paradigm in the MHD (Bv) picture. Adopting the similar logic used in Subsect. 6.1, another possible explanation is deceleration of the convection through the change in ionospheric potential (Tanaka 2000b).

The 4th panel of Fig. 26 at $t=67.2$ min shows the pressure distribution at onset. At this time, the pressure peak in the plasma sheet is shifted to the region inside $x=-10$ Re. In the next panel at $t=72.6 \mathrm{~min}$, the pressure peek appears at $x=-8 \operatorname{Re}$ and remains there afterward. In this way, the position of peak plasma pressure shifts from $x=-12$ to $x=-8 \mathrm{Re}$ in less than $6 \mathrm{~min}$. These variations show the signature of the dipolarization and injection occurring in the inner magnetosphere. From the development of plasma sheet configuration in Fig. 26, the plasmoid formation is estimated to be after the onset, with the NENL formed around $x=-30 \mathrm{Re}$. The 4th panel of Fig. 27 shows that before the onset the neutral line moves to the near-earth region around $x=-32$ Re. During the expansion phase, this NENL persists in the midtail, generating fast earthward flows inside $x=-32 \mathrm{Re}$ and tailward flows outside.

\subsection{Growth Phase and Distant-Tail Neutral Line}

The upper panel of Fig. 28 shows a snapshot of the magnetospheric configuration during the growth-phase interval together with the ionospheric convection. While open field lines generated from the southward IMF through the dayside reconnection wrap the outer part of the lobe, the core part still consists of open field lines generated from the northward IMF. This situation is quite similar to the formation process of cross-tail $\mathrm{Z}$ as shown in Sect. 6.3. In this thinning stage, therefore, the reclosure process in the plasma sheet is not effective, since it is still controlled by the remnants of northward IMF. From numerical results shown in Sect. 5, it is well understood that the magnetic topology in the core part inhibits an effective reconnection in the plasma sheet because two bundles of lobe open field lines from the northern and southern hemispheres are twisted around the $x$ axis limiting anti-parallelism.

The flaring angle theory is often proposed to explain plasma sheet thinning (Baker et al. 1996). Under this theory, the open magnetic field lines accumulate on the nightside due to the transport after reconnection on the dayside, producing a larger flaring angle. Therefore, the lobes are more strongly compressed by solar wind pressure, which leads to increased lobe magnetic pressure. This increased lobe pressure compresses the plasma sheet and thins it. It is obvious that this theory relies upon an extension of the magnetospheric image based on localized MHD balance or single-particle description. In this paper, on the contrary, plasma population regimes are generated from the convection (see Sect. 3). Starting from this concept, another growth-phase mechanism can be extracted from the magnetic topology during the growth phase shown in Fig. 28, by reconsidering the principle controlling the convection as shown before.

Before considering the growth phase mechanism, the structure of distant neutral line must also be reconsidered. Figure 29 shows again the structure of merging cell under the 
Fig. 29 Structure of merging cell convection generating the distant neutral line. This figure shows type 2 (upper) and type 3 (lower) field lines over a wide area as far as $x=-150 \mathrm{Re}$. Upper left and right insets show the footpoint on the ionosphere and cross point with $y z$ plane at $x=-70$ Re. Dashed line shows the inclined plasma sheet at $x=-70 \mathrm{Re}$. A peculiar feature is seen in the development of type 3 field line. Initially, it proceeds from dusk to dawn but it goes back to again toward dusk (skew back process)

\section{Merging cell convection and distant neutral line}

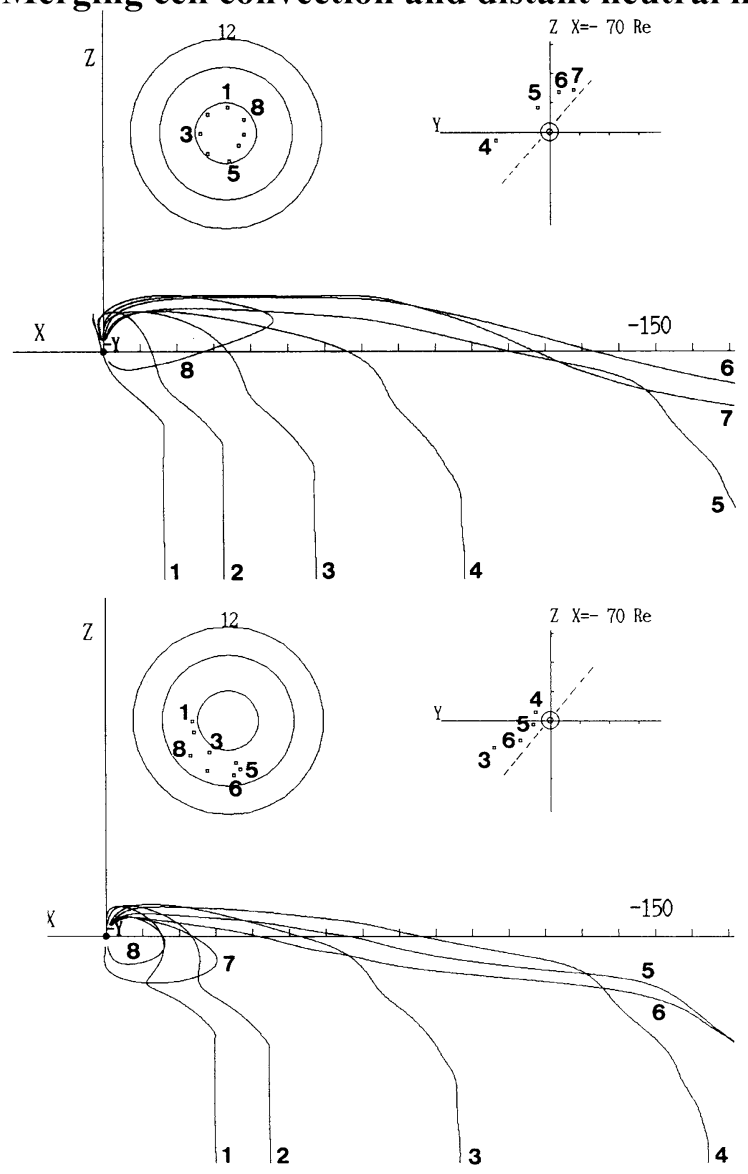

northward IMF condition with non-zero IMF $B_{y}$. This figure shows the structure of magnetic field lines (type 2 and type 3 ) constructing the merging cell, over wider area than in Figs. 16 and 17. In addition to the magnetic configuration, the crossing points of magnetic field lines with the $y z$ plane at $x=-70 \mathrm{Re}$ are shown in the upper right inset together with footpoints of field lines on the northern ionosphere in the upper left inset. In the upper right inset, dashed line shows the inclined plasma sheet at $x=-70 \mathrm{Re}$. After entering the lobe, the type 2 field line (upper panel) proceeds toward dawn in the midst of the lobe, and finally, the type 2 field line approaches the plasma sheet near the dawn flank. The type 3 field (lower panel) proceeds toward dawn for a while in the region nearest to the plasma sheet, but it goes back toward dusk and approaches the dusk plasma sheet. Corresponding to this motion, curvature of type 3 field is reversed in the deep tail region. As shown in Sect. 5, the twist structure of type 2 and 3 field lines prevents the anti-parallelism. Results in Fig. 29 show the twist back process in the tail that cancels the anti-parallelism and realizes the parallelism. There is a possibility that the twist back process makes the position of tail neutral line to be located at more distant region. This is a natural understanding for the formation mechanism of the distant neutral line. 
Fig. 30 Schematic diagram showing the growth phase convection. The dashed arrows show the regions of enhanced convection, while solid arrows show the force balance. The divergence thinning for the substorm growth phase is seen from this figure. Flow divergence is equal to inductive electric field, and consequently it generates a change in magnetic configuration

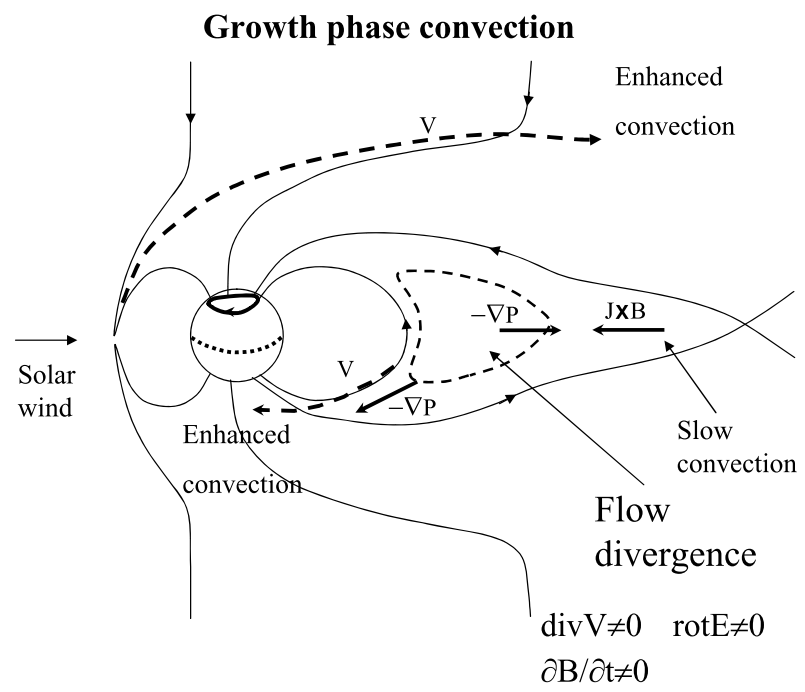

The growth phase mechanism under such a distant neutral line structure is shown in Fig. 30. With the development of the dayside reconnection, the magnetospheric convection from the dayside to the nightside is enhanced. However, the distant-tail neutral line does not immediately disappear (Fig. 28), so that the plasma sheet backflow does not immediately increase in the magnetospheric convection. In the ionosphere, on the other hand, a large magnitude of B prevents the occurrence of a divergent flow. This non-divergent nature of the ionospheric flow together with the fact that the magnetospheric dynamo driving the region-1 FAC is distributed in the near-earth cusp and mantle is responsible for a quick response of the ionospheric convection to a change in the IMF (see Sect. 3). The convection in the inner magnetosphere is connected to the quickly responding two-cell convection in the ionosphere as shown in Fig. 30, and consequently the flow from the inner edge of the plasma sheet must divert around both sides of the earth to the dayside.

Thus, magnetosphere and the ionosphere tend to have different timescales in their response to the change in IMF. However, the ionosphere does not need to incorporate the entire situation of the magnetosphere. In this case magnetospheric convection needs to be seen from the ionosphere as a shear flow. The magnetospheric convection can generate a shear flow against the ionosphere by creating a divergent flow in the plasma sheet that squeezes out the plasma already accumulated in the plasma sheet to dayside. This situation causes the outgoing sunward flux from the plasma sheet to exceed the supply of flux to the plasma sheet from the distant-tail neutral line. As schematically shown in Fig. 30, the loss of closed magnetic flux from the near-earth tail prevails over the supply of closed magnetic flux to the near-earth tail. This net loss (drain) of closed magnetic flux results in the plasma sheet thinning in the growth phase. In the diagrams presented in Fig. 9, the divergent flow corresponds to the condition $\partial B / \partial t \neq 0$, and thinning is a natural consequence of this condition.

\subsection{Substorm Onset}

No matter how large in magnitude the M-I disturbance may be, it would not be regarded as a substorm if it starts gradually. A major objective of substorm study is to find an explanation for the appearance of discontinuity at onset. A dipolarization event that definitely corresponds to the condition $\partial B / \partial t \neq 0$ characterizes onset, and reveals the convergent property 
of magnetospheric convection (Fig. 9). Naturally, this convergent motion is not projected onto the ionosphere. As a consequence, the magnetosphere needs to generate such motion as that confined to the magnetosphere.

The substorm onset occurs as an abrupt change of the magnetospheric configuration in the near-earth tail. The 5th panels in Figs. 26 and 27 show pressure and $-V_{x}$ distributions after the onset illustrating the appearance of the high-pressure region in the inner magnetosphere, fast earthward flow and the formation of the NENL in the midtail. From Fig. 28, this change is identified as the dipolarization (onset).

Figure 31 shows pressure and $V_{x}$ distributions along the $-x$ axis in the near-earth and midtail before and after the onset. Before the onset $(t<70 \mathrm{~min})$, the strongest $-\nabla P$ force acts in the region between $x=-10$ and $-20 \mathrm{Re}$. As a result, earthward convection is obstructed at $x=-14 \mathrm{Re}$. In addition, a gradual formation of NENL is seen at $x=-33 \mathrm{Re}$ before the onset. At $t=70 \mathrm{~min}$, a sudden change of pressure profile is seen to start just like a transition from one state to another. After the onset $(t>70 \mathrm{~min})$, the peak position in the pressure distribution shows a rapid inward movement. The pressure peak abruptly moves further inward to $x=-8 \mathrm{Re}$. At the same time, the convection flow intrudes into the inner magnetosphere inside $x=-10 \mathrm{Re}$ increasing in magnitude. Through these transition processes, a new stress balance is achieved in the near-earth plasma sheet in which recovered magnetic tension is balanced by newly established pressure inside $x=-10 \mathrm{Re}$. This pressure change is, in turn, a result of energy conversion from magnetic energy to internal energy caused by the pumping effect of convection associated with the recovery of magnetic tension (see Sect. 3). The fastest earthward flow in the plasma sheet appears after about $5 \mathrm{~min}$ from the onset. Then, tailward flow increases its speed. After $t=75.3 \mathrm{~min}$, the NENL begins to gradually retreat downtail.

The NENL model in the lower panel of Fig. 32 (Baker et al. 1996) explains flow convergence (dipolarization) as a pileup of fast flow from the NENL. Therefore, the motion is in a fast wave mode, in which both the magnetic field and fluid are similarly compressed. This produces tailward pressure that is balanced by the earthward dynamic pressure. This structure is the same as that for the bow shock in Fig. 6, and the negative $\mathbf{J} \cdot \mathbf{E}$ generates a dawnward current. This current is considered to correspond to the current wedge. The substorm discontinuity in this model results from the rapid development of NENL. The reconnection must be an instability in this case, and is regarded as a result of kinetic processes. In this model, the onset proceeds from the tail to the inner magnetosphere (steps 1, 2, and 3 in Fig. 32, bottom panel). The other weakness of this model lies in the discordance in the brightening order of quiet arcs (which proceed from the equator to polar direction) that are already present before the onset. Furthermore, it fails to explain the fact that substorm onset is triggered by a northward turning of IMF (Lyons et al. 1997).

Since $\mathbf{J}$ is not calculated directly from evolutionary equations, but alternatively calculated by $\mathbf{B}$ through Ampere's law, the dipolarization and $\mathrm{CD}$ are, under the $\mathbf{B v}$ paradigm, different representations of same physical processes. In contrast to the NENL model, the CD model attributes the substorm discontinuity to the $\mathrm{CD}$ (dipolarization). In the CD model, disturbance produced as a result of CD propagates to the tail and triggers the NENL (Fig. 32, top panel) (Lui 1996). In this model, the onset proceeds from the inner magnetosphere to the tail (steps 1, 2, and 3 in Fig. 32, top panel). The CD is a kinetic process and is also an instability. Supporters of this model associate the severe magnetic oscillations accompanying dipolarization with non-MHD processes, and seem to believe that there may even be a global slip between the magnetic field and plasma (non-ideal MHD process) (Lui 1996). Therefore, this model is constructed under the EJ paradigm. The CD model is consistent with the brightening order of the quiet arcs. 
Fig. 31 Time sequence of $P$ and $-V_{x}$ distributions along the $-x$ axis around the onset. Pressure and velocity is normalized by solar wind pressure (47 $\mathrm{pPa})$ and solar wind sound velocity $(53 \mathrm{~km} / \mathrm{sec})$. Sudden transition is observable between $t=70.0 \mathrm{~min}$ and $72.6 \mathrm{~min}$

\section{State transition at the onset}
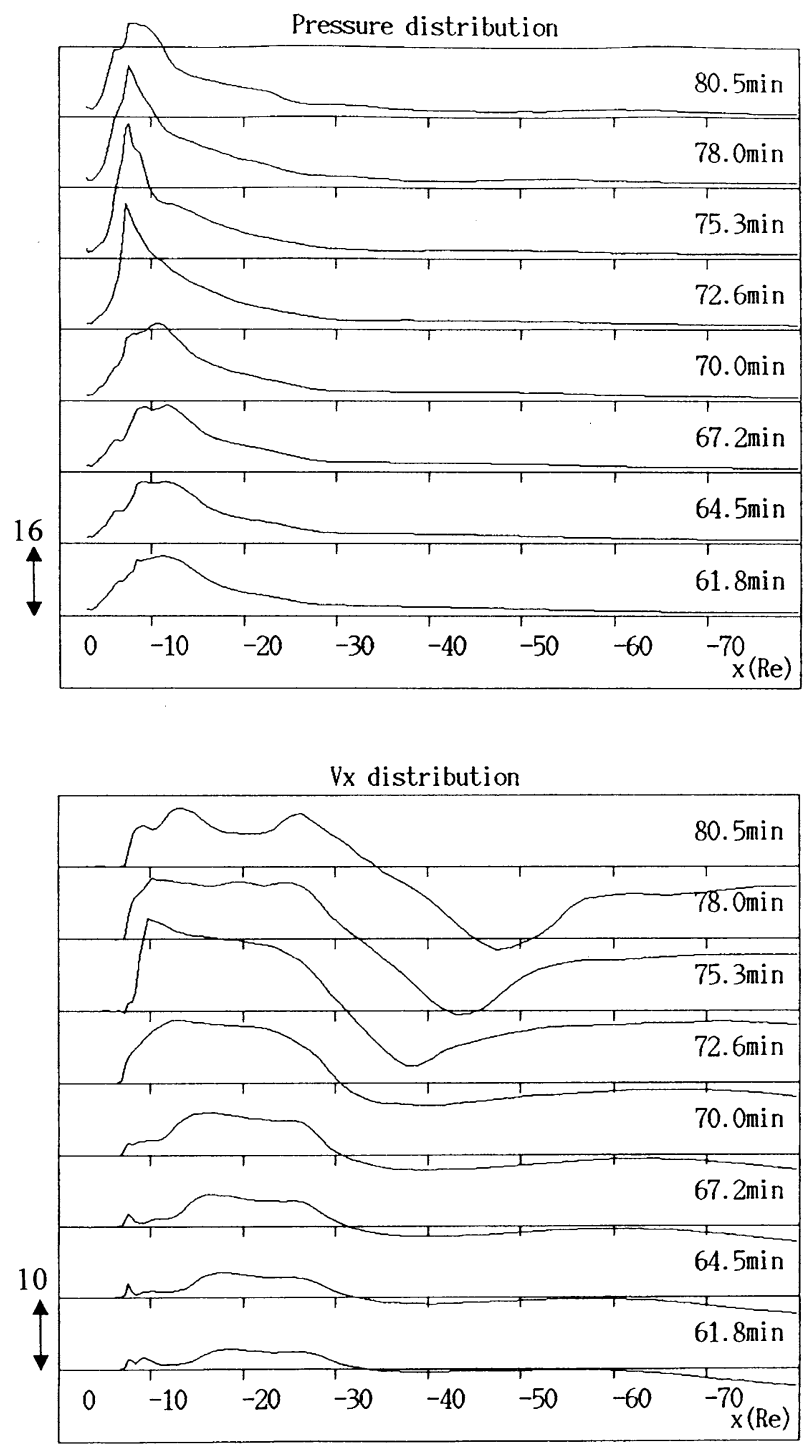

\subsection{Mechanical Balance Associated with the Onset}

In the near-earth region, the plasma sheet is filled with plasma even during the growth phase. This plasma supports the $\mathbf{J} \times \mathbf{B}$ force in the $x$-direction and constructs the convection system including the plasma population regimes (see Sect. 3). In the region between -12 and $-30 \mathrm{Re}$, the distribution of plasma pressure changes little even after the onset, whereas a drastic change of plasma pressure occurs in the near-earth region inside $-12 \mathrm{Re}$ (Fig. 31). These results strictly coincide with observations given by Kistler et al. (1992). In the result shown in Fig. 26, therefore, the NENL is never a floating object in the space but an interaction system with the supporting plasma. In this situation, the convection in the plasma 
Fig. 32 Schematic diagrams showing two different concepts for substorm initiation. Top and bottom panels represent near-earth initiation and mid-tail initiation models, respectively. If a primary cause for the onset is assumed, the resulting effects occur in sequence as show by numbers. Solid arrows show the force balance. In the lower panel (mid-tail initiation), dominance of kinetic energy is unavoidable in the near earth region. On the contrary, onset is generated retaining $\mathbf{J} \times \mathbf{B}=-\nabla P$ in the near-earth tail initiation
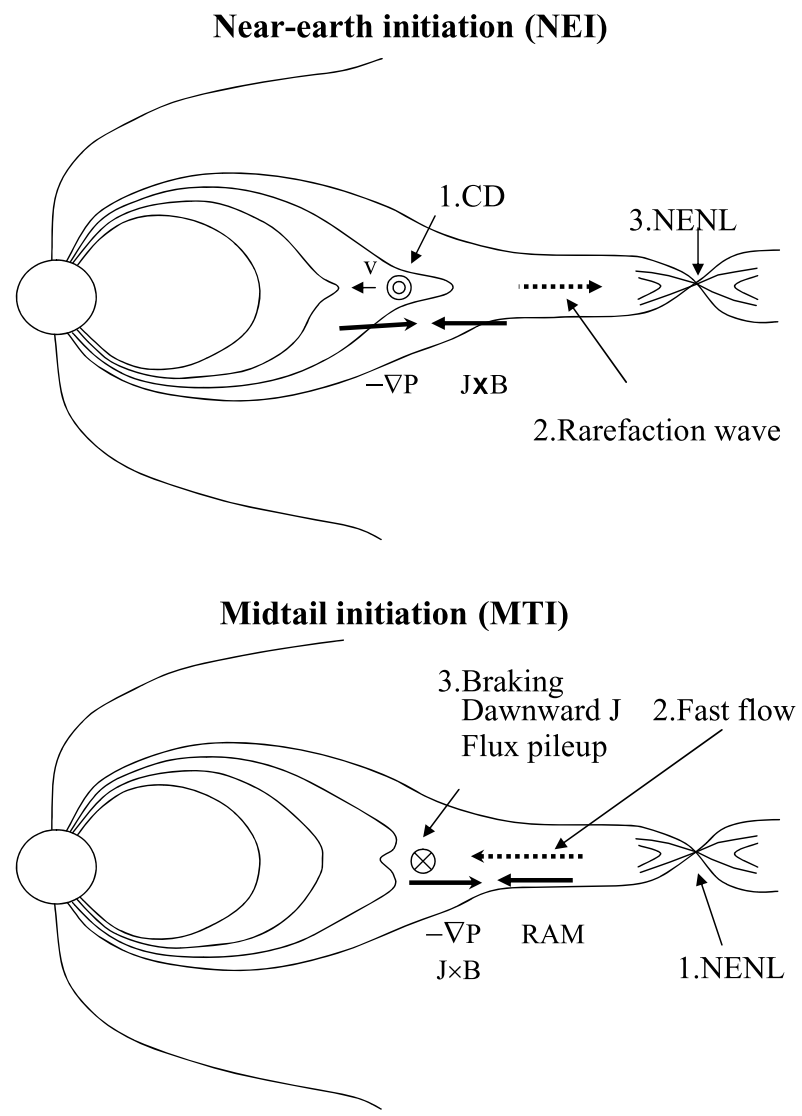

sheet is in the subsonic regime and nearly incompressible. Actually, flow surrounding the slant slow shock is almost incompressible (Lee 1995). In the incompressible convection system, the flow configuration must change as a whole. Only a portion of the convection cannot become fast, since in such a case flow convergence is required at the front of the fast flow and flow divergence is required at the rear. Briefly speaking, a fluid element in the incompressible flow cannot move until a fluid element in the front position moves aside.

The concept of state transition has also been suggested by Atkinson (1991), followed by Sitnov et al. (2000) and Tanaka (2000b). The state transition model by Tanaka (2000b) regards the onset as extension of the development process of convection. During the growth phase, the balance between plasma pressure and magnetic pressure in the $z$-direction is the dominant element in the force balance with rather flat pressure distribution in the $x$ direction. The dipolarization is considered to be an escape from this state with a restoration of tension resulting from the shrinkage of the elongated magnetic field. In the dipolarization process, the restored tension balances in the $x$-direction with increased pressure which is, in turn, a result of the pumping effect associated with the plasma sheet convection which is directed from low-pressure region to high-pressure region against $-\nabla P$ force (see Sect. 3). The energy conversion between the magnetic and internal energies acts as a kind of selfadjustment system. In other words, tension confines the plasma and enables convergence of 
the convection, allowing convection motion to be driven even when the magnetosphere and ionosphere are partially out of sync.

It is natural to observe earthward flow before the onset (Fig. 31) because under the frozenin condition the magnetic configuration cannot change without preceding flow which carries the magnetic field. The kinetic aspect of reconnection may provide the pathway between the states. However, the discontinuous behavior at onset is primarily attributed to a realization of initial (high energy metastable) and destination (low energy metastable) states in the MHD regime. The sudden dipolarization is interpreted as a self-organization phenomenon in a nonlinear system (Tanaka 2000b). In this view, the onset is a bifurcation process from a tail-like state to a dipolar state through the change of force-balance direction from the $z$-direction to $x$-direction.

The pressure increase inside $10 \mathrm{Re}$ can occur without a compression of magnetic field, if plasma moves along the field line. This kind of motion corresponds to the slow mode variation in Fig. 9. Recently such motion fas been confirmed from the satellite observations (Nakamizo and Iijima 2003). The development of the pressure distribution within the plasma sheet shown in Fig. 31 is also similar to those observations by Kistler et al. (1992), and generates a maximum within $10 \mathrm{Re}$ after onset. These variations are rapid, and within $1 \mathrm{~min}$, a transition occurs in the pressure distribution from the growth phase to the expansion phase. In this process, the dipolarization is a reinforcement of tension and never a relaxation to the potential field (Tanaka 2000b).

In the NENL model of the substorm, on the contrary, the magnetic energy is converted to the kinetic energy through the NENL formation. The ground onset is attributed to the braking of this kinetic energy in the inner magnetosphere (Baker et al. 1996). Figure 33 shows distribution of internal and kinetic energy during the initial state, growth phase and expansion phase. Apparently the NENL model is inconsistent with the results shown in Fig. 33. On the other hand, the state transition model can generate the substorm onset without the dominance of kinetic energy everywhere in the tail. It generates the onset along a natural extension of the incompressible convection in the M-I coupling system.

The NENL model and the CD model both assume that there must be a central player initiating the onset. In contrast, there is no central player in the state transition model. The state transition model resembles the economic model of major depressions, the Ising model for magnetization, or the avalanche model for substorms (Chapman et al. 1998). These models are all based on cooperative phenomena, and no central players exist. Instead, in these models, many similar elements coexist and interact with one another. The state transition of the interacting systems corresponds to the conditions of a major depression and of magnetization. The onset of a substorm is the occurrence of state transition in the interacting system, as shown in Fig. 31. A substorm does not involve as many elements as the economic model or the model for magnetic bodies. However, all elements in substorm have their characteristic topologies. Since the conditions differ significantly from those of a typical complex system found in economic models and models for magnetic bodies, we refer the M-I system as a compound system.

The state transition in the near-earth region explains quite well why the onset starts from the equatorward-most pre-onset arc. In general, the state transition requires the existence of multiple solutions for one boundary condition. As a consequence, it requires a nonlinearity of the system. On the other hand, the relation between the NENL solution and inflow and outflow boundary conditions is in one-to-one correspondence which generates no state transition (Lee 1995).

The state-transition model can also explain the triggering effect caused by the northward turning of IMF. The deceleration of ionospheric convection reduces the flux exiting the 


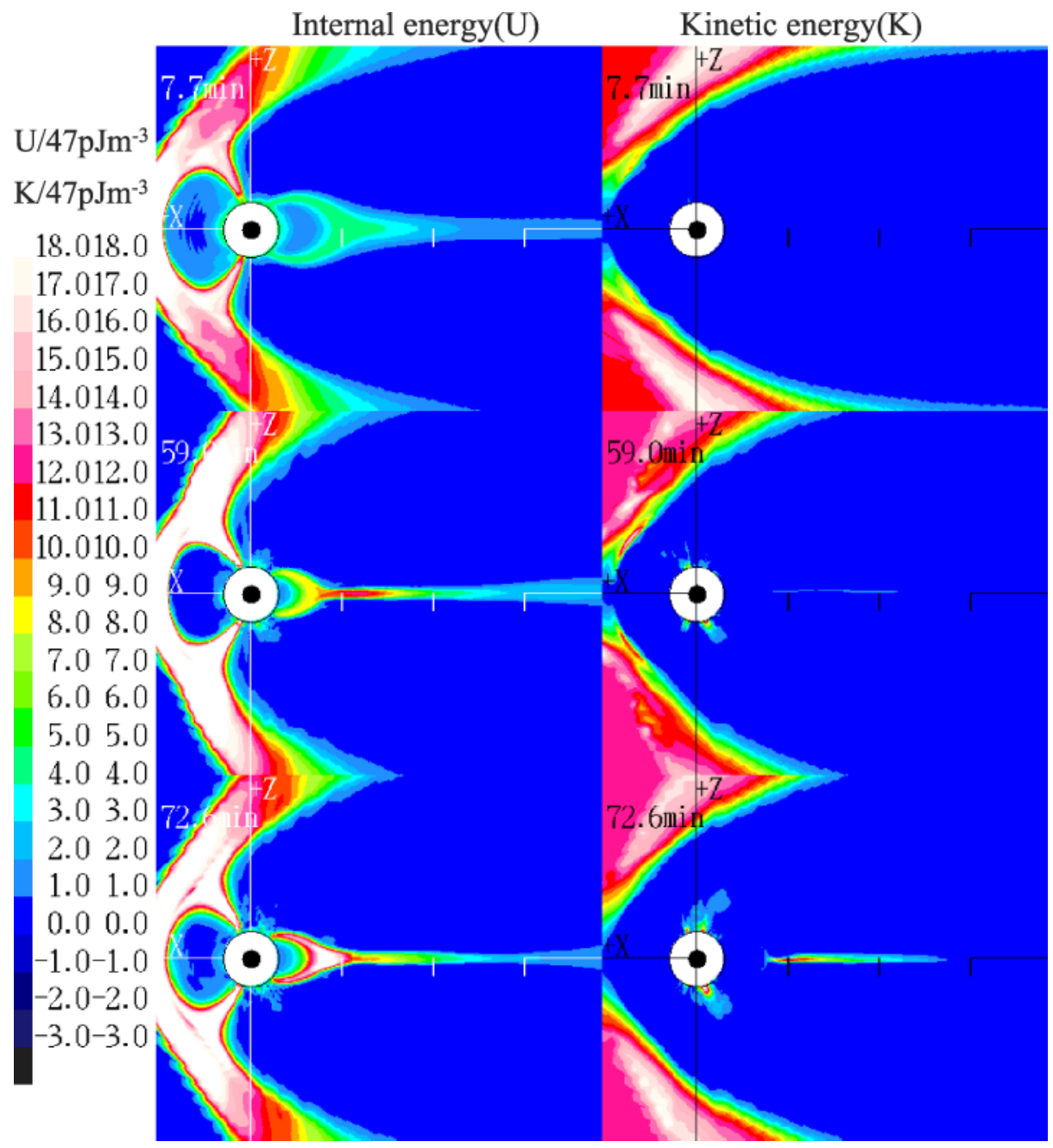

Fig. 33 Distributions of internal energy (left) and kinetic (right) energy in the noon-midnight meridian plane. From top to bottom three panels show initial, growth, and expansion phases. In the magnetosphere, internal energy prevails over kinetic energy in all cases. In the solar wind, on the contrary, kinetic energy prevails over internal energy. The unit for color codes is $47 \mathrm{pJ} \mathrm{m}^{-3}$

plasma sheet to the dayside, creating a region prone to flow convergence. The triggering effect by northward turning of IMF is also explained by Lyons (1995). However, Lyons' theory attempts to explain the triggering effect as originating from the deceleration of convection caused by the penetration of the weakened solar wind electric field into the magnetosphere. Since this model uses $\mathbf{E}$ as a primary variable in MHD regime, it leads to the mistake of $\mathbf{E J}$ paradigm pointed out by Parker (2000).

The difference between the state-transition model and NENL model can be understood from the analogy of waterpower electric generation. The left panel of Fig. 34 shows a water fall-like system. In this system, kinetic energy is the main source for the generation of electric power. However, waterpower electric plants in practical use do not have such a con- 
Fig. 34 Water electric analogy for energy generation of substorm onset. Electromagnetic energy is generated from kinetic energy (left) or from internal energy (right)

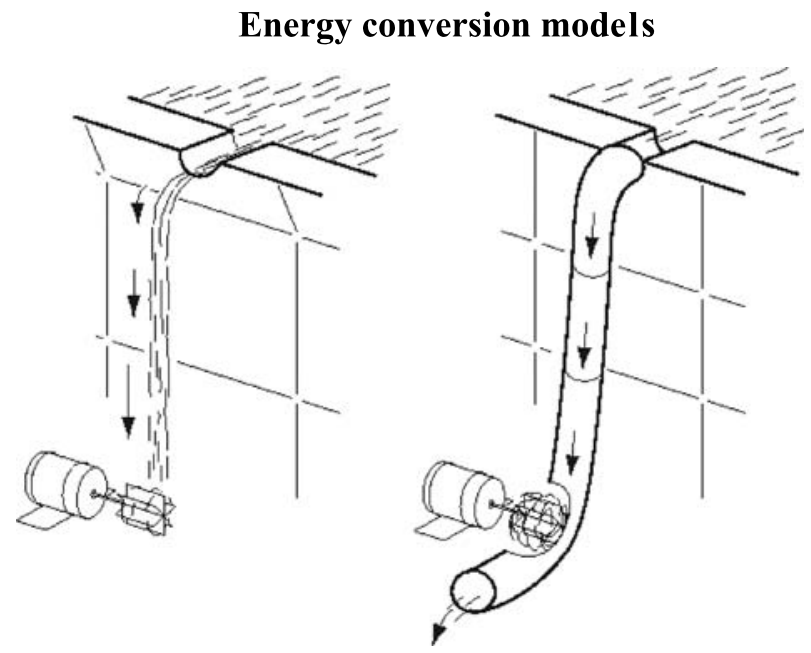

figuration. They send water through water pipes as shown in the right panel of Fig. 34. Here, flow is incompressible and internal energy (pressure) acts a major role for the generation of the electric power. The energy conversion in the state transition model is in analogy with this system.

\subsection{Pseudo Breakup and SMC}

The pseudo breakup and steady magnetospheric convention (SMC, or convection bay) are the phenomena resembling the substorm but they are grouped into different categories. The pseudo breakup exhibits a similar morphology to the substorm until onset. However, it does not accompany a poleward expansion or a westward traveling surge (WTS) of aurora, and terminates before changes are propagated to the whole magnetosphere (Pulkkinen et al. 1998). Similar to the substorm, the pseudo breakup shows a discontinuous behavior accompanied by Pi2.

In general, the SMC continues under the steady southward IMF whose magnitude is less than $5 \mathrm{nT}$. The magnitude of the magnetic disturbance in an SMC is comparable to that of the substorm, but an SMC progresses to an expansion-phase-like state without displaying a clear discontinuity, or onset (Yahnin et al. 1994; Sergeev et al. 1996). In the SMC, a strong, stationary two-cell ionospheric convection is realized in the polar ionosphere, while the magnetospheric status during the SMC shows the signature of recovery phase.

In the state-transition model, pseudo breakup can be interpreted as follows. Normally, state transition is initiated in the inner magnetosphere with the extinction of the distant-tail neutral line shown in Figs. 28 and 29, and then the substorm proceeds to the expansion phase. However, if state transition occurs before the extinction of the distant-tail neutral line shown in Figs. 28 and 29, discontinuity similar to onset can be realized without the formation of successive NENL due to the existence of core structure. This process results in a failure to proceed to the expansion phase, and instead, the event ends as the pseudo breakup.

If convection is promoted without a state transition or after the state transition has been initiated, and the state of no distant-tail neutral line is maintained, then the event is the SMC. In this way, the state-transition model provides simple explanations for the pseudo breakups and SMC. 


\section{Conclusions}

This paper has pursued how the global convection system constructs a compound system by which the magnetosphere and the ionosphere are self-consistently organized into a dynamically interacting system. The physical process underlying many magnetospheric phenomena can be clarified by examining how the convection structure shown in Fig. 10 is realized in a self-consistent manner when responding to given solar wind conditions. To clarify these problems, the structure of M-I system was analyzed in the numerical space as an energy conversion and consumption system. For the construction of a self-consistent configuration, not only the flows and current systems but also plasma population regimes must be coupled mutually through the dynamo formation process.

Observational confirmation of the linkage between elements in the compound system may be quite difficult, since the observations must be done simultaneously over many elements constructing the system. Especially, observational determination of magnetospheric configuration is the most difficult problem due to the one point nature of satellite observation. However, we got a proper confirmation of the correctness of a numerically reproduced $\mathrm{M}-\mathrm{I}$ system from the comparison of 2D ionospheric convection patterns.

The concept of the compound system and the requirement for a self-consistent configuration are important not only for disturbance phenomena such as the substorm, the SMC, and the pseudo breakup, but also for apparently unique phenomena such as the SC, the TCV, the theta aurora, and the FTE. Consequently, the physics of the M-I coupling system are described in terms of the physics of compound systems that considers the importance of self-consistency in the whole system, the role of system topology, and the possibility of state transitions as cooperative phenomena in the entire system.

In this paper some new terms are introduced from the consideration of compound system structure for realistic topology. They include (1) $\mathbf{J} \cdot \mathbf{E}$ overhang structure in the cusp dynamo process (Sect. 3), (2) $\mathbf{J} \cdot \mathbf{E}$ two-ribbon structure in the cusp dynamo process (Sect. 3), (3) merging concentration zone in the dayside reconnection process (Sect. 5), (4) exchange cell for oblique northward IMF condition (Sect. 5), (5) MI transient cell for the convection in the SC main phase (Sect. 6), (6) colliding convection and cross-tail $\mathrm{Z}$ for theta aurora generation (Sect. 6), (7) skew back mechanism for distant neutral line formation (Sect. 7), (8) drain thinning for the substorm growth phase (Sect. 7), and (9) state transition model for the substorm onset (Sect. 7). These concepts give alternative explanations to popular theoretical scenarios in magnetospheric physics that have been believed until now.

In the popular magnetospheric physics, many unresolved problems tend to be reduced to the special behavior of the reconnection. For instance, FTE is attributed to a pulsed reconnection, substorm onset is reduced to a sudden progress of reconnection, distant neutral line is reduced to a slow reconnection, and pseudo breakup is attributed to a mid-course termination of reconnection. Where, many critical problems are replaced by specific characteristics of reconnection without considering 3D topology. Apparently, excessive trust of Dungey (1961) is the starting point of this labyrinth.

No mater how the local plasma process is clarified, it does not result in the final understanding the M-I system. We cannot be exempted from considering the topological effects in the M-I system. From the viewpoint of this paper, global configuration changes that enable instability conditions are more essential for the determination of global configuration than the instability itself. For instance, how to realize anti-parallel or parallel magnetic configurations through the distortion of magnetic field by convection is a more dominant factor than the internal structure of reconnection for the generation of global change in the S-M-I 
system. With these topological effects, the models treating the M-I system cannot be reduced to a low-dimension system. From this fact, the global simulation becomes essential for understanding the physics of M-I compound system.

\section{References}

S.-I. Akasofu, Planet. Space Sci. 12, 273 (1964)

S.-I. Akasofu, S. Chapman, C.-I. Meng, J. Atmos. Terr. Phys. 27, 1275 (1965)

S.-I. Akasofu, J. Geophys. Res. 101, 10531 (1996)

V. Angelopoulos, W. Baumjohann, C.F. Kennel, F.V. Coroniti, M.G. Kivelson, R. Pellat, R.J. Walker, H. Luhr, G. Paschmann, J. Geophys. Res. 97, 4027 (1992)

T. Araki, Planet. Space Sci. 25, 373 (1977)

T. Araki, in Solar Wind Sources of Magnetospheric Ultra-Low-Frequency Waves. Geophys. Monogr. Ser., vol. 81, ed. by M.J. Engebretson, K. Takahashi, M. Scholer (AGU, Washington, 1994), p. 183

G. Atkinson, J. Geophys. Res. 83, 1089 (1978)

G. Atkinson, in Magnetospheric Substorms. Geophys. Monogr. Ser., vol. 64, ed. by J.R. Kan, T.A. Potemra, S. Kokubun, T. Iijima (AGU, Washington, 1991), p. 191

G. Atkinson, D. Hutchison, J. Geophys. Res. 83, 725 (1978)

W.I. Axford, C.O. Hines, Can. J. Phys. 39, 1433 (1961)

D.N. Baker, T.I. Pulkkinen, V. Angelopoulos, W. Baumjohann, R.L. McPherron, J. Geophys. Res. 101, 12975 (1996)

W. Baumjohann, G. Paschmann, H. Luhr, Geophys. Res. Lett. 17, 45 (1990a)

W. Baumjohann, G. Paschmann, H. Luhr, J. Geophys. Res. 95, 3801 (1990b)

K. Birkeland, The Norwegian Polaris Expedition 1902-1903, vol. 1 (Aschhoug, Oslo, 1908), Sect. 1

M. Blanc, Geophys. Res. Lett., 5, 203 (1978)

J. Bonnell, R.C. Elphic, S. Palfery, R.J. Strangeway, W.K. Peterson, D. Klumpar, C.W. Carlson, R.E. Ergun, J.P. McFadden, J. Geophys. Res. 104, 12669 (1999)

J.L. Burch, P.H. Reiff, J.D. Menietti, R.A. Heelis, W.B. Hanson, S.D. Shawhan, E.G. Shelley, M. Sugiura, D.R. Weimer, J.D. Winningham, J. Geophys. Res. 90, 1577 (1985)

P.F. Bythrow, R.A. Heelis, W.B. Hanson, R.A. Power, R.A. Hoffman, J. Geophys. Res. 86, 5577 (1981)

G. Caudal, M. Blanc, Rev. Geophys. 26, 819 (1988)

S.-W. Chang, J.D. Scudder, J.B. Sigwarth, L.A. Frank, N.C. Maynard, W.J. Burke, W.K. Peterson, E.G. Shelley, R. Friedel, J.B. Blake, R.A. Greenwald, R.P. Lepping, G.J. Sofko, J.-P. Villain, M. Lester, J. Geophys. Res. 103, 17367 (1998)

S. Chapman, V.C.A. Ferraro, J. Geophys. Res. 36, 77 (1931)

S. Chapman, J. Geophys. Res. 40, 349 (1935)

S.C. Chapman, N.W. Watkins, R.O. Dendy, P. Helander, G. Rowlands, Geophys. Res. Lett. 25, 2397 (1998)

C.R. Clauer, Y. Kamide, J. Geophys. Res. 90, 1343 (1985)

S.W.H. Cowley, Radio Sci. 8, 903 (1973)

S.W.H. Cowley, in From the Sun: Auroras, Magnetic Storms, Solar Flares, Cosmic Rays, ed. by S.T. Suess, T. Tsurutani (AGU, Washington, 1998), p. 13

S.W.H. Cowley, in Magnetospheric Current Systems, Geophys. Monogr. Ser., vol. 118, ed. by S. Ohtani et al. (AGU, Washington, 2000), p. 91

S.W.H. Cowley, M. Lockwood, Ann. Geophys. 10, 103 (1992)

T.E. Cravens, Physics of Solar System Plasma. Cambridge Atmos. Space Sci. Ser. (Cambridge University Press, Cambridge, 1997)

N.U. Crooker, J. Geophys. Res. 84, 951 (1979)

N.U. Crooker, J. Geophys. Res. 90, 12104 (1985)

N.U. Crooker, J. Geophys. Res. 93, 7338 (1988)

N.U. Crooker, J. Atmos. Terr. Phys. 52, 1123 (1990)

N.U. Crooker, J.G. Lyon, J.A. Fedder, J. Geophys. Res. 103, 9143 (1998)

W.D. Cumming, A.J. Dessler, J. Geophys. Res. 72, 1007 (1967)

J.A. Cumnock, J.R. Sharber, R.A. Heelis, M.R. Hairston, J. Geophys. Res. 102, 17489 (1997)

J.A. Cumnock, J.R. Sharber, R.A. Heelis, L.G. Blomberg, G.A. Germany, J.F. Spann, W.R. Coley, J. Geophys. Res. 107, A7 (2002). doi:10.1029/2001JA009126

D.L. Dezeeuw, S. Sazykin, R.A. Wolf, T.I. Gombosi, A.J. Ridley, G. Toth, J. Geophys. Res. 109, A12219 (2004). doi:10.1029/2003JA010366

J.W. Dungey, Phys. Rev. Lett. 6, 47 (1961) 
R.D. Elphinstone, J.S. Murphree, L.L. Cogger, Rev. Geophys. 34, 169 (1996)

G.M. Erickson, R.W. Spiro, R.A. Wolf, J. Geophys. Res. 96, 1633 (1991)

D.H. Fairfield, L.J. Cahill Jr., J. Geophys. Res. 71, 155 (1966)

G.J. Fasel, J. Geophys. Res. 100, 11891 (1995)

J.A. Fedder, S.P. Slinker, J.G. Lyon, J. Geophys. Res. 103, 14799 (1998)

J.A. Fedder, S.P. Slinker, J.G. Lyon, C.T. Russell, J. Geophys. Res. 107 A5 (2002). doi:10.1029/ 2001JA000025

L.A. Frank, J.D. Craven, J.L. Burch, J.D. Winningham, Geophys. Res. Lett. 9, 1101 (1982)

E. Friis-Christensen, Y. Kamide, A.D. Richmond, S. Matsushita, J. Geophys. Res. 90, 1325 (1985)

S. Fujita, T. Tanaka, T. Kikuchi, K. Fujimoto, K. Hosokawa, M. Itonaga, J. Geophys. Res. 108(A12), 1416 (2003a). doi:10.1029/2003JA009763

S. Fujita, T. Tanaka, T. Kikuchi, K. Fujimoto, M. Itonaga, J. Geophys. Res. 108(A12), 1417 (2003b). doi:10.1029/2003JA009407

N. Fukushima, Rep. Ionos. Space Res. Jpn. 30, 35 (1976)

T.I. Gombosi, K.G. Powell, B. van Leer, J. Geophys. Res. 105, 13141 (2000)

L. Harang, J. Geophys. Res. 51, 353 (1946)

M. Harel, R.A. Wolf, P.H. Reiff, R.W. Spiro, W.J. Burke, F.J. Rich, M. Smiddy, J. Geophys. Res. 86, 2217 (1981)

K. Hashimoto, T. Kikuchi, Y. Ebihara, J. Geophys. Res. 107(A11) 1337 (2002). doi:10.1029/2001JA009228

J.P. Heppner, N.C. Maynard, J. Geophys. Res. 92, 4467 (1987)

T. Iijima, in Magnetospheric Current Systems. Geophys. Monogr. Ser., vol. 118, ed. by S. Ohtani et al. (AGU, Washington, 2000), p. 107

T. Iijima, T.A. Potemra, J. Geophys. Res. 81, 2165 (1976)

T. Iijima, T.A. Potemra, L.J. Zanetti, P.F. Bythrow, J. Geophys. Res. 89, 7441 (1984)

R.K. Jaggi, R.A. Wolf, J. Geophys. Res. 78, 2852 (1973)

Y. Kamide, W. Sun, S.-I. Akasofu, J. Geophys. Res. 101, 99 (1996)

J.R. Kan, W.J. Burke, J. Geophys. Res. 90, 4171 (1985)

K.A. Keller, M. Hesse, M. Kuznetsova, L. Rastatter, T. Moretto, T.I. Gombosi, D.L. DeZeeuw, J. Geophys. Res. 107 A7 (2002). doi:10.1029/2001JA000060

H. Khan, S.W.H. Cowley, Ann. Geophys. 17, 1306 (1999)

T. Kikuchi, J. Geophys. Res. 91, 3101 (1986)

T. Kikuchi, H. Luhr, T. Kitamura, O. Saka, K. Schlegel, J. Geophys. Res. 101, 17161 (1996)

T. Kikuchi, H. Luehr, K. Schlegel, H. Tachihara, M. Shinohara, T.-I. Kitamura, J. Geophys. Res. 105, 23251 (2000)

T. Kikuchi, S. Tsunomura, K. Hashimoto, K. Nozaki, J. Geophys. Res. 106, 15555 (2001)

L.M. Kistler, E. Mobius, W. Baumjohann, G. Paschmann, J. Geophys. Res. 97, 2973 (1992)

M.G. Kiverson, D.J. Southwood, J. Geophys. Res. 96, 1661 (1991)

A. Kullen, Geophys. Res. Lett. 27, 73 (2000)

G. Le, G. Lu, R.J. Strangeway, R.F. Pfaff, Jr., J. Geophys. Res. 107 A12 (2002). doi:10.1029/2001JA007546

L.C. Lee, in Physics of Magnetopause. Geophys. Monogr. Ser., vol. 90, ed. by P. Song et al. (AGU, Washington, 1995), p. 139

M. Lockwood, S.W.H. Cowley, P.E. Sandholt, U.P. Lovhaug, J. Geophys. Res. 100, 7613 (1995)

R.E. Lopez, A.T.Y. Lui, J. Geophys. Res. 95, 8009 (1990)

A.T.Y. Lui, E.W. Hones Jr., F. Yasuhara, S.-I. Akasofu, S.L. Bame, J. Geophys. Res. 82, 1235 (1977)

A.T.Y. Lui, J. Geophys. Res. 101, 13067 (1996)

A.T.Y. Lui, in Magnetospheric Current Systems. Geophys. Monogr. Ser., vol. 118, ed. by S. Ohtani et al. (AGU, Washington, 2000), p. 31

A.T.Y. Lui, Rev. Geophys. 39, 535 (2001)

L.R. Lyons, Rev. Geophys. 30, 93 (1992)

L.R. Lyons, J. Geophys. Res. 100, 19069 (1995)

L.R. Lyons, G.T. Blanchard, J.C. Samson, R.P. Lepping, T. Yamamoto, T. Moretto, J. Geophys. Res. 102, 27039 (1997)

R.L. McPherron, M.P. Aubry, C.T. Russell, P.J. Coleman, J. Geophys. Res. 78, 3068 (1973)

J.J. Moses, G.L. Siscoe, R.A. Heelis, J.D. Winningham, J. Geopys. Res. 93, 9785 (1988)

D.L. Murr, W.J. Hughes, Geophys. Res. Lett. 28, 2145 (2001)

A. Nakamizo, T. Iijima, J. Geophys. Res. 108(A7) 1286 (2003). doi:10.1029/2002JA009400

T. Nagai, K. Takahashi, H. Kawano, T. Yamamoto, S. Kokubun, A. Nishida, Geophys. Res. Lett. 25, 2991 (1994)

P.T. Newell, D.G. Sibeck, Geophys. Res. Lett. 20, 1719 (1993)

P.T. Newell, C.-I. Meng, Science 270, 1338 (1995) 
A. Nishida, T. Ogino, in New Perspective on the Earth's Magnetotail. Geophys. Monogr. Ser., vol. 105, ed. by A. Nishida et al. (AGU, Washington, 1998), p. 61

T. Obara, T. Mukai, H. Hayakawa, A. Nishida, K. Tsuruda, S. Machida, H. Fukunishi, J. Geophys. Res. 98, $11153(1993)$

T. Obayashi, A. Nishida, Space Sci. Rev. 8, 3 (1968)

T. Ogino, R.J. Walker, M. Ashour-Abdalla, J.M. Dawson, J. Geophys. Res. 91, 10029 (1986)

S. Ohtani, K. Takahashi, T. Higuchi, A.T.Y. Lui, H.E. Spence, J.F. Fennell, J. Geophys. Res. 103, 4671 (1998)

S. Ohtani, T. Higuchi, J. Geophys. Res. 105, 25317 (2000)

C.J. Owen, S.W.H. Cowley, Planet. Space Sci. 35, 467 (1987)

E.N. Parker, Astrophys. J. 128, 644 (1958)

E.N. Parker, J. Geophys. Res. 101, 10587 (1996)

E.N. Parker, in Magnetospheric Current Systems. Geophys. Monogr. Ser., vol. 118, ed. by S. Ohtani et al. (AGU, Washington, 2000), p. 1

T.I. Pulkkinen, D.N. Baker, M. Wiltberger, C. Goodrich, R.E. Lopez, J.G. Lyon, J. Geophys. Res. 103, 14847 (1998)

P.H. Reiff, J.L. Burch, J. Geophys. Res. 90, 1595 (1985)

A.D. Richmond, Y. Kamide, J. Geophys. Res. 93, 5741 (1988)

A.J. Ridley, G. Lu, C.R. Clauer, V.O. Papitashvili, J. Geophys. Res. 102, 14563 (1997)

A.J. Ridley, G. Lu, C.R. Clauer, V.O. Papitashvili, J. Geophys. Res. 103, 4023 (1998)

A.J. Ridley, K.C. Hansen, G. Toth, D.L. De Zeeuw, T.I. Gombosi, K.G. Powell, J. Geophys. Res. 107(A10), 1290 (2002). doi:10.1029/2001JA000253

A.S. Rodger, S.W.H. Cowley, M.J. Brown, M. Pinnock, D.A. Simmons, Planet. Space Sci. 32, 1021 (1984)

J.M. Ruohoniemi, R.A. Greenwald, J. Geophys. Res. 101, 21743 (1996)

C.T. Russell, in Critical Problems of Magnetosphereic Physics, ed. by E.R. Dyer (Inter-Union Comm. on Sol.-Terr. Phys. Secr., Nat. Acad. of Sci., Washington, 1972), p. 1

C.T. Russell, R.C. Elphic, Space Sci. Rev. 22, 681 (1978)

V.A. Sergeev, R.J. Pellinen, T.I. Pulkkinen, Space Sci. Rev. 75, 551 (1996)

K.D. Siebert, G.L. Siscoe, J. Geophys. Res. 107 A7 (2002). doi:10.1029/2001JA000237

G.L. Siscoe, Planet. Space Sci. 14, 947 (1966)

G.L. Siscoe, N. Maynard, J. Geophys. Res. 96, 21071 (1991)

G.L. Siscoe, N.U. Crooker, G.M. Erickson, B.U.O. Sonnerup, K.D. Siebert, D.R. Weimer, W.W. White, N.C. Maynard, in Magnetospheric Current Systems. Geophys. Monogr. Ser., vol. 118, ed. by S. Ohtani et al. (AGU, Washington, 2000), p. 41

G.L. Siscoe, G.M. Erickson, B.U.O. Sonnerup, N.C. Maynard, K.D. Siebert, D.R. Weimer, W.W. White, J. Geophys. Res. 106, 13015 (2001)

G.L. Siscoe, N.U. Crooker, K.D. Siebert, J. Geophys. Res. 107(A10), 1321 (2002). doi:10.1029/ 2001JA009179

M.I. Sitnov, A.S. Sharma, K. Papadopoulos, D. Vassiliadis, J.A. Valdivia, A.J. Klimas, D.N. Baker, J. Geophys. Res. 105, 12955 (2000)

S.P. Slinker, J.A. Fedder, W.J. Hughes, J.G. Lyon, Geophys. Res. Lett. 26, 3549 (1999)

S.P. Slinker, J.A. Fedder, D.J. McEwen, Y. Zhang, J.G. Lyon, Phys. Plasma 8, 1119 (2001)

P. Song, C.T. Russell, J. Geophys. Res. 97, 1411 (1992)

B.U.O. Sonnerup, J. Geophys. Res. 85, 2017 (1980)

D.J. Southwood, J. Geophys. Res. 82, 5512 (1977)

P. Stauning, C.R. Clauer, T.J. Rosenberg, E. Friis-Christensen, R. Sitar, J. Geophys. Res. 100, 7567 (1995)

D.P. Stern, Rev. Geophys. 15, 156 (1977)

D.P. Stern, Rev. Geophys. 21, 125 (1983)

K. Takahashi, L.J. Zanetti, R.E. Lopez, R.W. McEntire, T.A. Potemra, K. Yumoto, Geophys. Res. Lett. 14, 1019 (1987)

T. Tamao, Geophysics 17, 43 (1965), Sci. Rep. Tohoku Univ., Series 5

T. Tanaka, J. Geophys. Res. 86, 11335 (1981)

T. Tanaka, Geophys. Res. Lett. 13, 1399 (1986)

T. Tanaka, J. Geophys. Res. 98, 17251 (1993)

T. Tanaka, J. Comput. Phys. 111, 381-389 (1994)

T. Tanaka, J. Geophys. Res. 100, 12057 (1995)

T. Tanaka, in Magnetospheric Research with Advanced Techniques. COSPAR Colloquium Ser., vol. 9, ed. by R.L. Xu, A.T.Y. Lui (Pergamon, 1998a), p. 133

T. Tanaka, Earth Planets Space 50, 259 (1998b)

T. Tanaka, J. Geophys. Res. 104, 14683 (1999)

T. Tanaka, in Magnetospheric Current Systems. Geophys. Monogr. Ser., vol. 118, ed. by S. Ohtani et al. (AGU, Washington, 2000a), p. 53 
T. Tanaka, J. Geophys. Res. 105, 2108 (2000b)

T. Tanaka, J. Geophys. Res. 106, 24505 (2001)

T. Tanaka, in Space Plasma Simulation. Lecture Notes in Physics, ed. by J. Buchner et al. (Springer, Berlin, 2003a), p. 275

T. Tanaka, J. Geophys. Res. 108(A8), 1315 (2003b). doi:10.1029/2002JA009668

T. Tanaka, K. Murawski, J. Geophys. Res. 102, 19805 (1997)

T. Tanaka, H. Washimi, J. Geophys. Res. 104, 12605 (1999)

T. Tanaka, H. Washimi, Science 296, 321 (2002)

T. Tanaka, T. Obara, M. Kunitake, J. Geophys. Res. 109, A09201 (2003). doi:10.1029/2003JA010271

A. Thorolfsson, J.C. Cerisier, M. Lockwood, P.E. Sandholt, C. Senior, M. Lester, Ann. Geophys. 18, 1054 (2000)

O.A. Troshichev, J. Atmos. Terr. Phys. 52, 1135 (1990)

V.M. Vasyliunas, in Particles and Fields in the Magnetosphere, ed. by B.M. MaCormac (Reidel, Hinham, 1970), p. 60

V.M. Vasyliunas, Ann. Geophys. 23, 1347 (2005a)

V.M. Vasyliunas, Ann. Geophys. 23, 2589 (2005b)

S. Vennerstrom, T. Moretto, L. Rastätter, J. Raeder, J. Geophys. Res. 110, A06205 (2005). doi:10.1029/ 2004JA010802

M. Watanabe, G.J. Sofko, D.A. Andre, T. Tanaka, M.R. Hairson, J. Geophys. Res. 109, A01215 (2004). doi:10.1029/2003JA009944

D.R. Weimer, J. Geophys. Res. 100, 19595 (1995)

W.W. White, G.L. Siscoe, G.M. Erickson, Z. Kaymaz, N.C. Maynard, K.D. Siebert, B.U.O. Sonnerup, D.R. Weimer, Geophys. Res. Lett. 25, 1605 (1998)

J.D. Winningham, W.J. Heikkila, J. Geophys. Res. 79, 949 (1974)

A. Yahnin, M.V. Malkov, V.A. Sergeev, R.J. Pellinen, O. Aulamo, S. Vennerstrom, E. Friis-Christensen, K. Lassen, C. Danielsen, J.D. Craven, C. Deehr, L.A. Frank, J. Geophys. Res. 99, 4039 (1994)

L. Zhang, R.L. Carovillano, in Auroral Plasma Dynamics. Geophys. Monogr. Ser., vol. 80, ed. by R.L. Lysak (AGU, Washington, 1993), p. 89 